\title{
Timing and nature of alluvial fan and strath terrace formation in the Eastern Precordillera of Argentina
}

\author{
Kathryn Hedrick ${ }^{\mathrm{a}, *}$, Lewis A. Owen ${ }^{\mathrm{a}}$, Thomas K. Rockwell ${ }^{\mathrm{b}}$, Andrew Meigs ${ }^{\mathrm{c}}$, \\ Carlos Costa ${ }^{\mathrm{d}}$, Marc W. Caffee ${ }^{\mathrm{e}}$, Eulalia Masana ${ }^{\mathrm{f}}$, Emilio Ahumada ${ }^{\mathrm{d}}$ \\ a Department of Geology, University of Cincinnati, Cincinnati, OH 45221, USA \\ b Department of Geological Sciences, San Diego State University, San Diego, CA 92182, USA

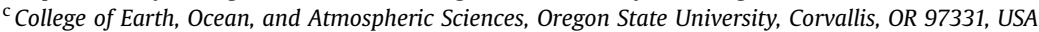 \\ ${ }^{\mathrm{d}}$ Department of Geology, Universidad Nacional de San Luis, 5700 San Luis, Argentina \\ e Department of Physics, Purdue University, West Lafayette, IN 47907, USA \\ ${ }^{\mathrm{f}}$ Department Geodinamica ia Geofisica, Universitat de Barcelona, Spain
}

\section{A R T I C L E I N F O}

\section{Article history:}

Received 7 January 2013

Received in revised form 25 April 2013

Accepted 7 May 2013

Available online 6 October 2013

\section{Keywords:}

Terrestrial cosmogenic nuclide dating

Optically stimulated luminescence dating

Climate change

Tectonics

Erosion rates

\begin{abstract}
A B S T R A C T
Sixty-eight ${ }^{10}$ Be terrestrial cosmogenic nuclide (TCN) surface exposure ages are presented to define the timing of alluvial fan and strath terrace formation in the hyper-arid San Juan region of the Argentine Precordillera. This region is tectonically active, and numerous fault scarps traverse Quaternary landforms. The three study sites, Marquesado strath complex, Loma Negra alluvial fan and Carpintería strath complex reveal a history of alluvial fan and strath terrace development over the past $\sim 225 \mathrm{ka}$. The Marquesado complex Q3 $3_{\mathrm{m}}$ surface dates to $\sim 17 \pm 3 \mathrm{ka}$, whereas the Loma Negra Q1 $1_{\ln }, \mathrm{Q}_{\ln }, \mathrm{Q}_{\ln }, \mathrm{Q} 4_{\mathrm{ln}}$, and $5_{\text {ln }}$ surfaces date to $\sim 24 \pm 3 \mathrm{ka}, \sim 48 \pm 2 \mathrm{ka}, \sim 65 \pm 13 \mathrm{ka}, \sim 105 \pm 21 \mathrm{ka}$, and $\sim 181 \pm 29 \mathrm{ka}$, respectively. The Carpintería complex comprises eight surfaces that have been dated and include the Q1 $\mathrm{c}$ $(\sim 23 \pm 3 \mathrm{ka}), \mathrm{Q}_{\mathrm{c}}(\sim 5 \pm 5 \mathrm{ka}), \mathrm{Q}^{3} \mathrm{a}_{\mathrm{c}}(\sim 25 \pm 12 \mathrm{ka}), \mathrm{Q} \mathrm{b}_{\mathrm{c}}(\sim 29 \pm 15 \mathrm{ka}), \mathrm{Q}_{\mathrm{c}}(\sim 61 \pm 12 \mathrm{ka}), \mathrm{Q}_{\mathrm{c}}$ $(\sim 98 \pm 18 \mathrm{ka}), \mathrm{Q6}_{\mathrm{c}}(\sim 93 \pm 18 \mathrm{ka})$, and $\mathrm{Q}_{\mathrm{c}}(\sim 212 \pm 37 \mathrm{ka}) .{ }^{10} \mathrm{Be} \mathrm{TCN}$ depth profile data for the Loma Negra alluvial fan complex and Carpintería strath terrace complex, as well as OSL ages on some Carpintería deposits, aid in refining surface ages for comparison with local and global climate proxies, and additionally offer insights into inheritance and erosion rate values for TCNs $\left(\sim 10 \times 10^{4}{ }^{10} \mathrm{Be}\right.$ atoms/g of $\mathrm{SiO}_{2}$ and $\sim 5 \mathrm{~m} \mathrm{Ma}^{-1}$, respectively). Comparison with other alluvial fan studies in the region show that less dynamic and older preserved surfaces occur in the Carpintería and Loma Negra areas with only younger alluvial fan surfaces preserved both to the north and south. These data in combination with that of other studies illustrate broad regional agreement between alluvial fan and strath terrace ages, which suggests that climate is the dominant forcing agent in the timing of terrace formation in this region.
\end{abstract}

(C) 2013 Elsevier Ltd. All rights reserved.

\section{Introduction}

Alluvial fans are one landform of a continuum - including strath terraces and talus slopes - related to mountain range-proximal erosion and deposition. Alluvial fans are pervasive and commonly well-preserved landforms that form in dynamic landscapes in a wide variety of geomorphic and climatic settings, and they are especially widespread in tectonically active regions (Blair and McPherson, 1994; Harvey et al., 2005). Factors controlling alluvial fan and strath terrace formation are complex and the efficacy and relative contributions of different geomorphic processes may vary

\footnotetext{
* Corresponding author.

E-mail address: kate.hedrick@gmail.com (K. Hedrick).
}

from one region to the next. In addition, the dominant depositional processes at work often change temporally and spatially on alluvial fans, resulting in complex deposits that may be difficult to interpret. The importance of determining the timing and rate of alluvial fan formation lies in their utility to be used for paleoenvironmental, tectonic and landscape studies. Determining the main driver of deposition might also allow for mountain-proximal landforms to be used as a proxy for major tectonic or climatic events.

Influences on the timing of alluvial fan surface formation is greatly debated, with some studies emphasizing that tectonics is the dominant forcing factor while other studies suggest climate dominates (Wells and McFadden, 1987; Frostick and Reid, 1989; Bull, 1991; Blair and McPherson, 1994; Ritter et al., 1995; Harvey et al., 1999a,b; Harvey and Wells, 2003). In addition, many 
environments include other factors which may affect the timing of alluvial fan surface formation; for example, Trowbridge (1911), Church and Ryder (1972) and Church et al. (1989) suggest that paraglacial processes are important factors in alluvial fan formation, whereas Williams (1973), Wasson (1977), and Dorn (2009) emphasize the importance of periglacial processes in cold environments. However, recent studies of Quaternary alluvial fan sequences suggest that although tectonics is significant in providing conditions suitable for alluvial fan development, climatic events may be more important in determining the timing and rate of alluvial fan surface formation (Ritter et al., 1995; c.f. Harvey et al., 2005). The current lack of understanding of the timing and nature of alluvial fan formation represents a noteworthy gap in the scientific knowledge which may be filled by quantitative studies of the timing of alluvial fan formation.

Although alluvial fans are common landforms worldwide, the best places to study alluvial fans are usually in arid or hyper-arid environments where alluvial fan surfaces are more readily preserved and the lack of vegetation helps makes them easy to study. Extensive research has been undertaken on alluvial fans worldwide for many years (Denny, 1965; Boothroyd and Nummendal, 1977; Bull, 1977; Kesel and Spicer, 1985; Kostaschuk et al., 1986; Kochel, 1990), however many of the previous studies have relied on relative dating techniques (e.g. surface characteristics, geomorphology of terraces, soil development) to define the ages of alluvial fan surfaces because the most common numerical dating method-radiocarbon geochronology-cannot generally be applied due to the paucity of organic material in arid or hyper-arid alluvial fans. In addition, another common numerical dating method, optically stimulated luminescence (OSL), although well-suited for the dating of sand, cannot always be readily used due to the coarse grained nature of most alluvial fan deposits. Recently, however, the development of terrestrial cosmogenic nuclide (TCN) surface exposure dating has allowed alluvial fans to be dated more readily (e.g. Owen et al., 2006; Frankel et al., 2007; Hall et al., 2008; Spelz et al., 2008; Frankel et al., 2011; Hall et al., 2012).

To explore the nature and timing of alluvial fan formation in a hyper-arid region, and to assess the relative importance of forcing factors for alluvial fan development, a series of alluvial fans and strath terrace surfaces in the foreland of the Eastern Precordillera of Argentina on the western outskirts of San Juan, $\sim 150 \mathrm{~km}$ north of Mendoza were examined. For the purposes of this paper we define the terms alluvial fan and strath terrace as follows: alluvial fans are a fan-shaped landform comprising thick $(>2 \mathrm{~m}$ ) sedimentary deposits and/or a lack of visible bedrock, whereas strath terraces are defined as landforms covered in thin $(<2 \mathrm{~m})$ sedimentary deposits or with visible underlying bedrock which lack the characteristic alluvial fan shape.

Fans and strath terrace deposits are well preserved in the forelands of the Eastern Precordillera. Determining the timing and mode of formation of alluvial fans and terraces in this region is important for both tectonic and paleoenvironmental studies. We map and date, using ${ }^{10} \mathrm{Be}$ TCN and OSL dating methods, multiple surfaces on three different alluvial fan-strath terrace complexes along the eastern front of the Sierra Chica de Zonda range near San Juan. Our study is compared to other alluvial fan and terrace studies in the Precordillera (Siame et al., 1997; Colombo et al., 2000; Siame et al., 2002; Schmidt et al., 2011, 2012) to explore whether climatic or tectonic events are most important in their formation along the Eastern Precordillera.

\section{Regional setting}

San Juan province is situated in the northwest-central region of Argentina and forms a portion of the western border with Chile, located above the southern Pampean flat-subduction segment of the Andes (Ramos et al., 2002). The province contains a wide variety of geomorphic settings with the tall, glaciated mountains of the High Andean Cordillera (Cordillera Principal and Cordillera Frontal), rising up to the peak of El Mercedario $6770 \mathrm{~m}$ above sea level (asl), which take up approximately the western third of the province. This is followed eastward by the significantly lowerelevation $(<3000 \mathrm{~m}$ asl) of the Precordillera before finally flattening along its eastern border into the Sierras Pampaneas broken foreland (Jordan et al., 1983; Ramos et al., 1986; Mpodozis and Ramos, 1989; von Gosen, 1992; Ramos et al., 2002), which are expressed as generally flat plains with isolated, fault-bounded mountains that rise up to $2500 \mathrm{~m}$ asl, but generally with a relative relief of $<700 \mathrm{~m}$ above the valley floor surrounded by bajada and strath surfaces. One such isolated mountain is Pie de Palo ( $\sim 3300 \mathrm{~m}$ asl), which borders the northeastern edge of San Juan city. The detailed study areas are located entirely within the eastern piedmont of the Eastern Precordillera, where the Quaternary Andean thrust front is located (Costa et al., 2000; Ramos et al., 2002; Costa et al., 2006; Meigs et al., 2006; Vergés et al., 2007).

Our field areas abut this portion of the Eastern Precordillera, which comprise the Sierra de Villicum (Marquesado alluvial fan) and the Sierra Chica de Zonda (Loma Negra and Carpintería alluvial fans). These ranges are bounded by the west-verging VillicumPedernal thrust fault, which trends approximately north-south along the western edge of both the Sierra de Villicum and the Sierra Chica de Zonda (Costa et al., 2000).

Alluvial fans and strath terraces form an extensive bajada along Chica de Zonda, stretching for $\sim 150 \mathrm{~km}$ from San Juan to Mendoza (Fig. 1). Study areas were chosen near the city of San Juan on the backlimb of the Villicum-Pedernal thrust (Fig. 1). Previous studies of alluvial fans in this region include those of Siame et al. (1997), Siame et al. (2002) and Colombo et al. (2000) $60 \mathrm{~km}$ to the north, $\sim 10 \mathrm{~km}$ north and $\sim 15 \mathrm{~km}$ northeast of our study areas, respectively. Schmidt et al. (2011) and Schmidt et al. (2012) also conducted studies on alluvial fans $\sim 80 \mathrm{~km}$ and $\sim 130 \mathrm{~km}$ to the south.

The region is tectonically active and has experienced many large earthquakes, notably Argentina's most disastrous earthquake: the 1944 San Juan $\mathrm{M}_{\mathrm{w}} 7$ earthquake, which destroyed $~ 80 \%$ of San Juan and surrounding areas (Bastías and Henríquez, 1984; Perucca and Paredes, 2002; Alvarado et al., 2007; Meigs and Nabelek, 2010) $\sim 20 \mathrm{~km}$ to the northeast of our study area. The Sierras Pampeanas to the east are affected by active east- and west-verging reverse faults with a thick-skinned origin (Kandinsky-Cade et al., 1985; Fielding and Jordan, 1988; Ramos et al., 2002; Costa et al., 2006; Meigs and Nabelek, 2010), whereas the central Precordillera to the west comprises thin-skinned east-vergent thrust faults (von Gosen, 1992). The eastern Precordillera acts as a transition zone (Fielding and Jordan, 1988; Zapata and Allmendinger, 1996) between these two tectonic environs and is characterized by thin-skinned westverging thrust faults shallower than $\sim 5 \mathrm{~km}$ depth (von Gosen, 1992) and reverse fault seismicity deeper than $40 \mathrm{~km}$ (Smalley et al., 1993). Studies undertaken by many researchers suggest that the crust beneath both the Eastern Precordillera and the Sierras Pampeanas is affected by active planar reverse faults below $5 \mathrm{~km}$ depth (Kandinsky-Cade et al., 1985; Smalley et al., 1993; Meigs et al., 2006; Gimenez et al., 2010; Meigs and Nabelek, 2010), whereas other researchers suggest that these deeper structures are secondary to fault movement (Vergés et al., 2007).

San Juan and the nearby study areas are located within the South American Arid Diagonal (Bruniard, 1982; Morales et al., 2009), a region believed to have been climatically sensitive to the shifts of the Pacific and Atlantic anticyclones from the late Pleistocene through the Holocene (Abraham et al., 2000). For the period of 1940-2007, mean annual precipitation in San Juan was $93 \mathrm{~mm}$ 


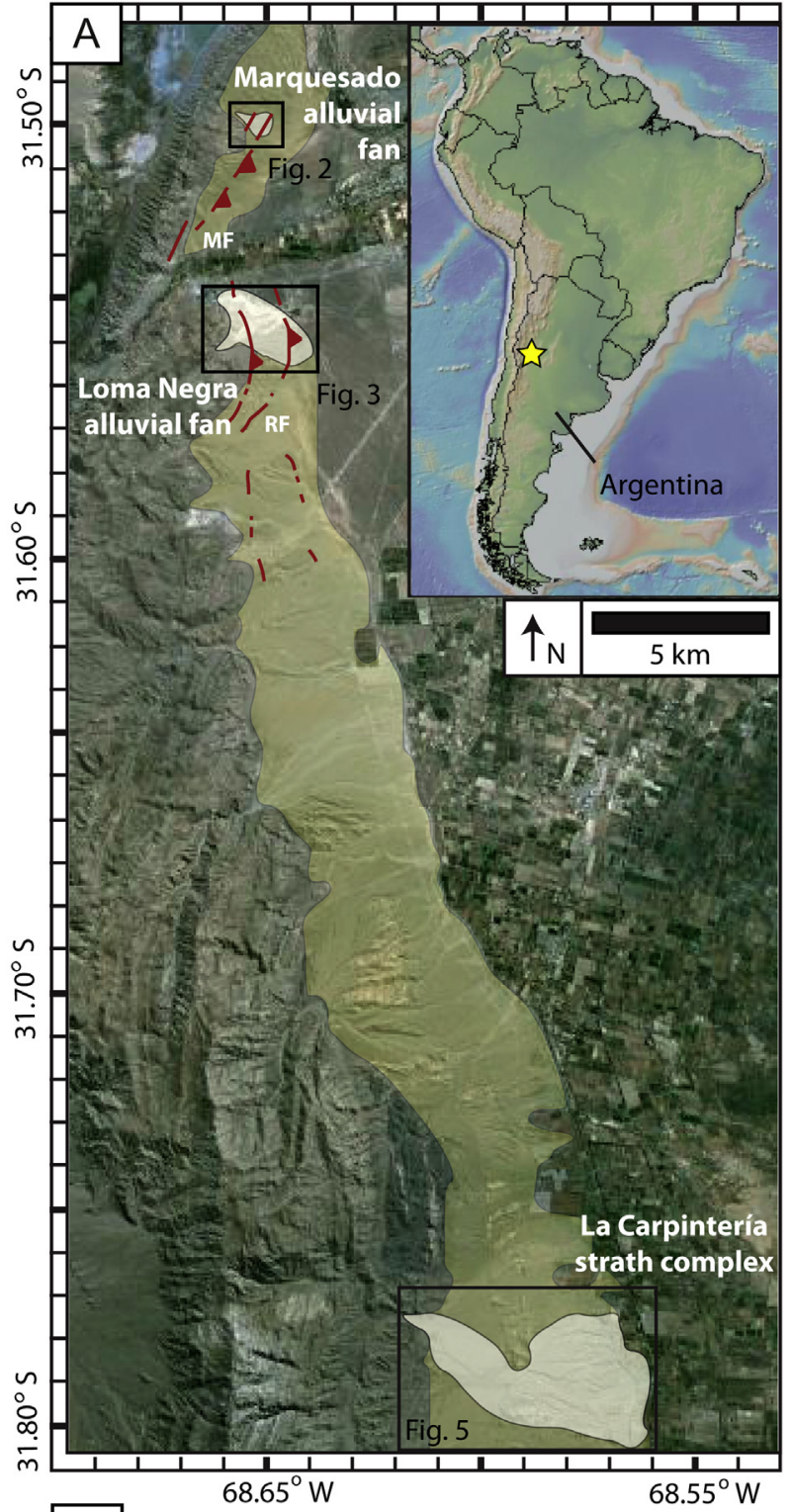

B

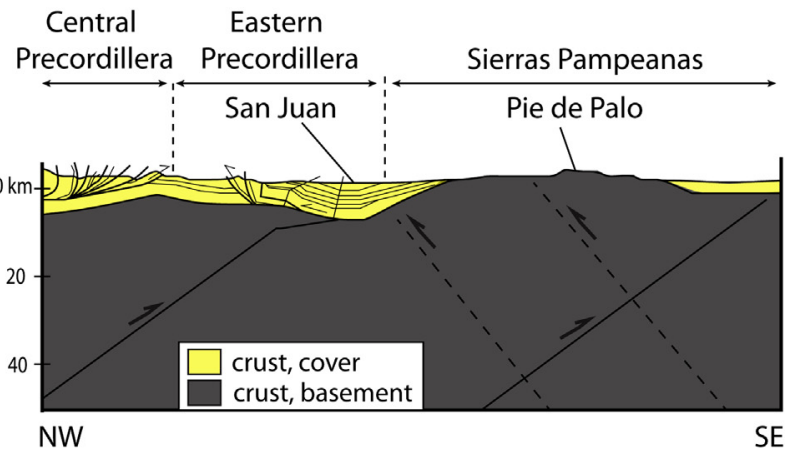

Fig. 1. A) Location of detailed study areas. Yellow star on the overview map marks the location of the main image. Note the extensive bajada (yellow) developed east of the mountain front. White areas are the areas investigated in this study. Red lines delineate the observed track of fault scarps on strath terrace and alluvial fan surfaces. $\mathrm{MF}=$ Marquesado Fault, RF = Rinconada Fault. Base image from GoogleEarth. B) Schematic section showing the general tectonic setting of the study area following Meigs (2012; pers. comm.). (For interpretation of the references to color in this figure legend, the reader is referred to the web version of this article.)
(SMN, 2007; Gimenez et al., 2010), classifying it as hyper-arid (growing period $<0$ days - FAO-IIASA Agro-ecological Zone, 2002; annual precipitation $<200 \mathrm{~mm}-\mathrm{FAO}, 2013$ ).

The San Juan region is affected by both the Atlantic and Pacific Ocean anticyclones (Capitanelli, 1972; Mancini et al., 2005); Atlantic wind currents lose much of their moisture as they travel westward over the continent, and Pacific winds precipitate a good deal of the moisture to the west in the higher mountain ranges of the Andes. Studies suggest that although the region's climate has fluctuated throughout the Holocene, over the past 6 ka the climate has generally remained hyper-arid to semi-arid within a generally stable South American Arid Diagonal (Mancini et al., 2005; Morales et al., 2009) and has maintained modern climatic conditions from $\sim 3$ ka to recent (Markgraf, 1983). Studies of dune migration at Medanos Grandes, $\sim 45 \mathrm{~km}$ west of San Juan, suggest climatic conditions similar to modern for the last $\sim 4$ ka (Tripaldi and Forman, 2007). This is also addressed in Tripaldi and Forman's (2007) study, which suggests a period of increased aridity generally matching the modern from $0-4 \mathrm{ka}$; this trend is also present in dune activity $\sim 200 \mathrm{~km}$ south of our study areas (Tripaldi et al., 2011) as discussed below, suggesting some similarities in climate over a broader region. Mehl and Zárate (2012) investigated the Quaternary alluvial record of piedmonts $\sim 200 \mathrm{~km}$ southwest of our study area, but did not attribute their findings to any specific climatic shifts.

Given the sensitivity of the spatially-constrained Arid Diagonal to changing anticyclone activity, however, climate proxies from outside of the Arid Diagonal may not be accurate for our study region, although there does seem to be some relationship to global climate patterns. Tripaldi et al. (2011) and Kröhling (1999a,b) investigated fluvial and aeolian deposits in the Central Pampas and Northern Pampas, respectively. Tripaldi et al. (2011) use OSL methods to determine that, from 58-24 ka, which correlates to MIS stage 3 , conditions were generally semi-arid, and shifted to more arid conditions during the last glacial maximum at 24-12 ka, which correlates to MIS stage 2. Kröhling (1999a,b) provides a climate record of generally dry conditions with a brief mesic period in that region from 8.5-6.4 ka. Pollen, lake level, and diatom studies have been undertaken to help determine the nature of climate fluctuations, and have similarly been focused to the far north or south of the study region (e.g. Markgraf, 1993; Gilli et al., 2001; Markgraf et al., 2003; Mancini et al., 2005; De Francesco et al., 2007). Many of these records address only short-term $(<8 \mathrm{ka})$ climate change.

\section{Methods}

\subsection{Field methods and sample collection}

Three study areas were examined near San Juan (Fig. 1), which we call the Marquesado strath terrace complex, the Loma Negra alluvial fan, and the Carpintería strath terrace complex. Alluvial fan and strath terrace surfaces and fault scarps at each study area were identified and mapped in the field aided by Google Earth imagery.

Mapping of alluvial fan and strath terrace surfaces was undertaken using high-quality aerial photographs from Google Earth (CNES/SPOT Image, 2012; Inav/Geosistemas SRL, 2012) and in the field on printed imagery. Surfaces of different age were designated numerically, with numbers increasing with age, such that a Q1 surface represents the active stream channel, with subsequent numbers (Q2 through Q5) indicating progressively older alluvial fan or terrace surfaces. A separate local chronology was developed for each study area and the surfaces were numbered, Q1 through Q7; subscripted letters designated after the terrace number distinguish each local sequence of surfaces. Marquesado, Loma Negra and Carpintería complexes are designated by the subscripted 
letters, $\mathrm{m}, \mathrm{ln}$, and c, respectively. On all surfaces, we described surface weathering features of both the alluvial fan and strath terrace surfaces and the sampled cobbles or boulders (e.g. the presence of desert varnish and development of desert pavement). At the Loma Negra site, limestone cobbles and boulders displayed pitting on their upper exposed surfaces; we measured the depth of five pits on fifteen cobbles for each surface $\left(\mathrm{Q} 2_{\ln }-\mathrm{Q} 5_{\mathrm{ln}}\right)$ to calculate an average pitting depth.

Samples for ${ }^{10} \mathrm{Be}$ TCN dating were collected from surfaces of different ages at the Loma Negra and Carpintería locations; only one surface was sampled at the Marquesado complex. Either surface cobbles or boulders (where present) or sediment from within pits were collected (see Table 1 for sample sizes). For surface sampling, multiple cobbles were collected from each surface. For depth profile pit samples, the depth of each pit was measured and samples were collected for ${ }^{10} \mathrm{Be}$ dating at $\sim 30 \mathrm{~cm}$ intervals down the exposed face (see Table 2 for exact depths). At each level, quartz-rich pebbles ( $\sim 2-5 \mathrm{~cm}$ long, primarily chert) were dug out of the pit walls until $>500 \mathrm{~g}$ ( $\sim 15-20$ pebbles) had been collected. Notable sedimentary and pedogenic features within the pit section were described. Locations were recorded using a GPS, and each boulder and pit was photographed. Where applicable, skyline measurements were recorded using a handheld inclinometer and compass for shielding corrections. In addition, bedrock, talus and active stream sediment samples were collected, as appropriate, to help examine the nature of erosion and inherited TCNs. The geologic setting of the sampling sites is described together with the description of the fan and terrace surfaces below.

\section{2. ${ }^{10}$ Be dating and erosion rate methods}

All the ${ }^{10}$ Be samples were processed in the Cosmogenic Nuclide Laboratory at the University of Cincinnati. All bedrock, boulder, cobble, and pebble samples ( 250-500 g) were crushed and sieved to obtain the $250-500 \mu \mathrm{m}$ grain size fraction for quartz separation. Bedrock and boulder samples were chipped off using a chisel and hammer, whereas cobble samples were crushed whole. Pebbles were collected from each level of depth profile pits and were restricted to $\sim 2-5 \mathrm{~cm}$ long- axis. For the catchment-wide erosion rate samples, the sediment was first sieved to obtain the $250-500 \mu \mathrm{m}$ fraction. The $500-1000 \mu \mathrm{m}$ fraction was then crushed and sieved to obtain the $250-500 \mu \mathrm{m}$ fraction, which was added to the first sieved fraction. Quartz separation, and the extraction and calcination of Be for all samples were conducted following the standard methods as described by Dortch et al. (2009; c.f. Kohl and Nishiizumi, 1992). All samples were measured on the Purdue (University) Rare Isotope Measurement (PRIME) Laboratory's accelerator mass spectrometer (AMS).

The conversion of ${ }^{10} \mathrm{Be} /{ }^{9} \mathrm{Be}$ ratios reported by PRIME Lab AMS to ${ }^{10} \mathrm{Be}$ concentrations follows ICN standards of Nishiizumi et al. (2007) and was calculated as described by PRIME (2010). ${ }^{10} \mathrm{Be}$ exposure ages were calculated using the CRONUS calculator (main calculator ver. 2.1 ; constants file ver. 2.21 ), which employs a ${ }^{10} \mathrm{Be}$ decay constant of $5.10 \pm 0.26 \times 10^{-7} \mathrm{yr}^{-1}$. The CRONUS calculator reports different exposure ages based on spallation scaling schemes as determined by Desilets and Zreda (2003), Desilets et al. (2006), Dunai (2001), Lifton et al. (2001), and Lal (1991)/Stone (2000). We report ages calculated using the time-dependent model of Lal (1991) and Stone (2000), which uses a reference ${ }^{10} \mathrm{Be}$ production rate of $4.39 \pm 0.37$ atoms $\mathrm{g}^{-1} \mathrm{yr}^{-1}$. This time-dependent model accounts for ${ }^{10} \mathrm{Be}$ production rate flux due to temporal changes in the geomagnetic field. Please see Balco et al. (2009) for a discussion of the merits and limitations of each scaling scheme.

${ }^{10} \mathrm{Be}$ concentrations for depth profiles and erosion rates (bedrock, talus, and basin) were calculated using the same procedure described above. For all depth profiles, ${ }^{10} \mathrm{Be}$ concentrations and sample depths were analyzed using Hidy et al.'s (2010; version 1.2) MATLAB code, which utilizes a Monte Carlo simulator to produce best-fit depth profile curves and provide estimates of erosion rate and inheritance. The simulation was run using a stochastic uniform density with depth between values of 2.2 and $2.5 \mathrm{~g} \mathrm{~cm}^{-3}$ and with a reference production rate of 4.39 atoms $\mathrm{g}^{-1} \mathrm{yr}^{-1}$, adjusted using Lal (1991) and Stone's (2000) time- independent scaling scheme. Although density values of $2.5 \mathrm{~g} \mathrm{~cm}^{-3}$ may seem high, all profiles contain beds which are dominantly large, quartz-rich boulders, and thus approach the density of solid rock. Additionally, density within the sediment profiles has likely changed over time with the development of soils. We feel that a density range of $2.2-2.5 \mathrm{~g} \mathrm{~cm}^{-3}$ is reasonable as they are comparable to the measurements and models of other researchers on similar alluvial fans (e.g. Braucher et al., 2009; Rodés et al., 2011) and also due to the highly variable but densely packed nature of these alluvial fan deposits.

Bedrock and talus slope erosion rates were also determined by the CRONUS erosion rate calculator. Catchment-wide erosion rate calculations require correction for the catchment production rate; changes in shielding patterns due to slope angle and attitude affect the production rate within a catchment, which is also affected by changing latitude and elevation of the catchment. To make this correction, we first isolated the $60 \mathrm{~m}$ resolution SRTM of the basin in ArcMap and imported the basin grid data into Schoenbohm's (2008; pers. comm.) MATLAB code to acquire the mean catchment-wide production rate. This code applies the scaling scheme of Lal (1991)/Stone (2000) on a pixel-by-pixel basis to determine corrections for shielding and latitude/elevation change. Once the mean catchment-wide production rate is measured, we calculated the erosion rate within the catchment using our known ${ }^{10} \mathrm{Be}$ concentration, production rate, and an attenuation length of $0.6 \mathrm{~m}$. Error is propagated using the square of errors.

\subsection{OSL dating methods}

OSL samples were collected using opaque PVC tubes with a $\sim 5 \mathrm{~cm}$ diameter. The tubes were then driven horizontally into the sediment surface with the use of a hammer until fully imbedded, and then carefully removed by scraping away sediment around them and then extracting the tubes from the pit wall. The tubes were kept horizontal at all times throughout this process. Once extracted, the open ends of the tubes were covered in aluminum foil and duct tape. If any remaining space was left in the tube before taping, it was filled with newspaper in order to secure the sediment of the tube and reduce the risk of sediment movement and mixing. Labeled tubes were transported from the field in darkroom bags in order to reduce any risk of light exposure. The OSL samples were processed at the Luminescence Dating Laboratory at the University of Cincinnati. All sample processing and measurements were undertaken in laboratory safelight conditions (cf. Spooner et al., 2000) to avoid the possibility of bleaching the luminescence in the quartz. OSL sample tubes were opened and $\sim 2-3 \mathrm{~cm}$ of sediment was removed from each end of the tube to isolate the middle plug of sediment, which is subject to the least risk of mixing and contamination with leached quartz. To determine the concentration of radioisotopes for dose-rate determination, $\sim 20 \mathrm{~g}$ of sediment from the middle plug was ground into a fine powder and sent to Activation Laboratories in Ontario, Canada for instrumental neutron activation analysis.

The remaining sediment from the middle of the tube was then processed to isolate the quartz: samples were sieved to separate 
Table

Terrestrial cosmogenic nuclide data for alluvial fan/strath terrace surface cobbles and boulders and ${ }^{10} \mathrm{Be}$ age results.

\begin{tabular}{|c|c|c|c|c|c|c|c|c|c|c|c|c|c|c|c|c|c|c|}
\hline $\begin{array}{l}\text { Sample name } \\
\text { and location }\end{array}$ & $\begin{array}{l}\text { Fan } \\
\text { terrace } \\
\text { number }\end{array}$ & $\begin{array}{l}\text { Latitude } \\
\left({ }^{\circ} \mathrm{N}\right)\end{array}$ & $\begin{array}{l}\text { Longitude } \\
\left({ }^{\circ} \mathrm{E}\right)\end{array}$ & $\begin{array}{l}\text { Altitude } \\
\text { (m asl) }\end{array}$ & $\begin{array}{l}\text { Boulder size } \\
\text { height/width/ } \\
\text { length }(\mathrm{cm})\end{array}$ & $\begin{array}{l}\text { Sample } \\
\text { lithology }\end{array}$ & $\begin{array}{l}\text { Quartz } \\
\text { mass (g) }\end{array}$ & $\begin{array}{l}\text { Be } \\
\text { carrier } \\
\text { (g) }\end{array}$ & $\begin{array}{l}\text { Be carrier } \\
\text { concentration } \\
(\mathrm{mg} / \mathrm{g})\end{array}$ & ${ }^{10} \mathrm{Be} / /^{9} \mathrm{Be}\left(10^{-13}\right)$ & $\begin{array}{l}\text { Sample } \\
\text { thickness } \\
(\mathrm{cm})\end{array}$ & $\begin{array}{l}\text { Shielding } \\
\text { correction }\end{array}$ & $\begin{array}{l}{ }^{10} \mathrm{Be} \\
\text { concentration } \\
\left(\text { atom/g } \mathrm{SiO}_{2}\right. \\
\left.\mathrm{SiO}_{2} \times 10^{5}\right)^{\mathrm{a}}\end{array}$ & $\begin{array}{l}\text { Lal (1991) and } \\
\text { Stone (2000) } \\
\text { time- } \\
\text { independent }\end{array}$ & $\begin{array}{l}\text { Desilets and } \\
\text { Zreda (2003) } \\
\text { and Desilets } \\
\text { et al. }(2006) \\
\text { age }(\mathrm{ka})^{\mathrm{a}, \mathrm{b}}\end{array}$ & $\begin{array}{l}\text { Dunai (2001) } \\
\text { age (ka) })^{\mathrm{a}, \mathrm{b}}\end{array}$ & $\begin{array}{l}\text { Lifton et al. } \\
(2005) \text { age } \\
(\mathrm{ka})^{\mathrm{a}, \mathrm{b}}\end{array}$ & $\begin{array}{l}\text { Lal (1991), } \\
\text { Stone (2000) } \\
\text { time- } \\
\text { dependent }\end{array}$ \\
\hline \multicolumn{19}{|l|}{ MARQUESADO } \\
\hline SJ135A & $\mathrm{Q} 3_{\mathrm{m}}$ & -31.50854 & -68.66819 & 795 & $3 / 4.5 / 6$ & Chert & 23.2660 & 0.3646 & 1.3540 & $8.748 \pm 0.282$ & 3 & 1 & $1.24 \pm 0.040$ & $17.7 \pm 1.7$ & $19.4 \pm 2.4$ & $18.5 \pm 2.3$ & $18.8 \pm 2.0$ & $17.5 \pm 1.6$ \\
\hline SJ135B & & -31.50854 & -68.66819 & 795 & $2.5 / 3.5 / 6$ & Chert & 20.9561 & 0.3597 & 1.3540 & $6.616 \pm 0.218$ & 2.5 & 1 & $1.027 \pm 0.034$ & $14.7 \pm 1.4$ & $16.2 \pm 2.0$ & $15.5 \pm 1.9$ & $15.7 \pm 1.6$ & $14.6 \pm 1.3$ \\
\hline SJ135C & & -31.50854 & -68.66819 & 795 & $3 / 4 / 5.5$ & Chert & 22.3400 & 0.3546 & 1.3540 & $7.459 \pm 0.274$ & 3 & 1 & $1.071 \pm 0.039$ & $15.3 \pm 1.4$ & $16.8 \pm 2.0$ & $16.1 \pm 2.0$ & $16.4 \pm 1.7$ & $15.2 \pm 1.4$ \\
\hline SJ135D & & -31.50854 & -68.66819 & 795 & $3 / 4.5 / 5.5$ & Chert & 13.3646 & 0.3624 & 1.3540 & $27.148 \pm 0.970$ & 3 & 1 & $6.660 \pm 0.238$ & $97.1 \pm 9.3$ & $97.6 \pm 12.3$ & $94.0 \pm 11.8$ & $94.2 \pm 10.1$ & $88.7 \pm 8.3$ \\
\hline SJ135E & & -31.50854 & -68.66819 & 795 & $3 / 6 / 7.5$ & Chert & 21.7068 & 0.3459 & 1.3540 & $11.208 \pm 0.366$ & 3 & 1 & $1.616 \pm 0.053$ & $23.1 \pm 2.2$ & $24.1 \pm 3.1$ & $23.8 \pm 2.9$ & $24.1 \pm 2.5$ & $22.5 \pm 2.0$ \\
\hline SJ135F & & -31.50854 & -68.66819 & 795 & $2.5 / 5 / 6$ & Chert & 21.1440 & 0.3669 & 1.3540 & $7.299 \pm 0.867$ & 2.5 & 1 & $1.146 \pm 0.136$ & $16.4 \pm 2.4$ & $17.9 \pm 3.0$ & $17.2 \pm 2.9$ & $17.4 \pm 2.7$ & $16.2 \pm 2.4$ \\
\hline \multicolumn{19}{|l|}{ LOMA NEGRA } \\
\hline SJ108 & $\mathrm{Q} 1_{\mathrm{ln}}$ & -31.56275 & -68.67313 & 851 & $50 / 25 / 30$ & Chert & 22.1203 & 0.3433 & 1.3540 & $23.588 \pm 1.103$ & 2.5 & 0.970 & $3.312 \pm 0.155$ & $47.0 \pm 4.7$ & $46.4 \pm 6.0$ & $44.7 \pm 5.7$ & $44.7 \pm 5.7$ & $42.6 \pm 4.2$ \\
\hline SJ109 & & -31.56406 & -68.67387 & 856 & $25 / 40 / 60$ & Chert & 22.5536 & 0.3481 & 1.3540 & $1.386 \pm 0.042$ & 2 & 0.911 & $0.194 \pm 0.006$ & $2.9 \pm 0.3$ & $3.4 \pm 0.4$ & $3.1 \pm 0.4$ & $3.4 \pm 0.3$ & $3.1 \pm 0.3$ \\
\hline SJ110 & & -31.56101 & -68.67181 & 827 & $40 / 40 / 60$ & $\begin{array}{l}\text { Limestone } \\
\text { with chert }\end{array}$ & 20.0770 & 0.3632 & 1.3540 & $11.518 \pm 0.322$ & 5 & 0.976 & $1.885 \pm 0.053$ & $27.2 \pm 2.5$ & $28.7 \pm 3.3$ & $27.6 \pm 3.4$ & $27.8 \pm 2.9$ & $26.1 \pm 2.3$ \\
\hline SJ111 & & -31.56077 & -68.67159 & 819 & $40 / 40 / 70$ & $\begin{array}{l}\text { Limestone } \\
\text { with chert }\end{array}$ & 22.8571 & 0.3479 & 1.3540 & $11.538 \pm 0.668$ & 6 & 0.988 & $1.589 \pm 0.092$ & $23.2 \pm 2.4$ & $248 \pm 3.3$ & $23.8 \pm 3.1$ & $24.0 \pm 2.8$ & $22.5 \pm 2.3$ \\
\hline SJ112 & & -31.55947 & -68.67107 & 802 & $25 / 35 / 50$ & $\begin{array}{l}\text { Limestone } \\
\text { with chert }\end{array}$ & 20.7371 & 0.3426 & 1.3540 & $5.200 \pm 0.217$ & 2 & 1 & $0.777 \pm 0.032$ & $11.0 \pm 1.1$ & $12.2 \pm 1.5$ & $11.7 \pm 1.5$ & $11.9 \pm 1.3$ & $11.0 \pm 1.0$ \\
\hline SJ117 & Q2 $\ln$ & -31.55965 & -68.66265 & 784 & $15 / 25 / 50$ & Chert & 27.8666 & 0.3635 & 1.3540 & $30.148 \pm 1.202$ & 4 & 1 & $3.558 \pm 0.142$ & & $52.2 \pm 6.6$ & $49.9 \pm 6.3$ & $50.0 \pm 5.4$ & $47.1 \pm 4.4$ \\
\hline SJ118 & & -31.55963 & -68.66255 & 782 & $15 / 30 / 45$ & $\begin{array}{l}\text { Limestone } \\
\text { with chert }\end{array}$ & 23.6773 & 0.3484 & 1.3540 & $26.748 \pm 0.655$ & 4 & 1 & $3.561 \pm 0.087$ & $52.2 \pm 4.8$ & $52.3 \pm 6.4$ & $50.0 \pm 6.1$ & $50.0 \pm 5.2$ & $47.2 \pm 4.2$ \\
\hline SJ119 & & -31.55976 & -68.66249 & 784 & $20 / 40 / 50$ & Chert & 23.7129 & 0.3448 & 1.3540 & $27.148 \pm 9.567$ & 5 & 1 & $3.572 \pm 1.259$ & $52.7 \pm 19.4$ & $52.9 \pm 19.9$ & $50.6 \pm 19.0$ & $50.6 \pm 18.8$ & $47.7 \pm 17.5$ \\
\hline SJ120 & & -31.55968 & -68.66287 & 787 & $10 / 25 / 27$ & Chert & 1.5575 & 0.3596 & 1.3540 & $23.558 \pm 0.574$ & 5 & 1 & $3.555 \pm 0.087$ & $52.4 \pm 4.8$ & $52.5 \pm 6.4$ & & & \\
\hline SJ121 & & -31.55975 & -68.66326 & 789 & $12 / 20 / 40$ & Chert & 21.0474 & 0.3648 & 1.3540 & $24.648 \pm 0.706$ & 3 & 1 & $3.865 \pm 0.111$ & $56.0 \pm 5.2$ & $56.7 \pm 7.0$ & 54.1 & $54.2 \pm 5.6$ & $50.7 \pm 4.6$ \\
\hline SJ122 & & -31.55980 & -68.66343 & 790 & $15 / 20 / 35$ & Chert & 22.7427 & 0.3460 & 1.3540 & $39.938 \pm 0.914$ & 4 & 1 & $5.497 \pm 0.126$ & $80.7 \pm 7.4$ & $81.7 \pm 10.0$ & $78.2 \pm 9.5$ & $78.4 \pm 8.1$ & $73.7 \pm 6.6$ \\
\hline SJ101 & $\mathrm{Q} 3 \mathrm{I}_{\mathrm{n}}$ & -31.55987 & -68.66148 & 789 & 25/30/55 & Chert & 23.7269 & 0.3437 & 1.3540 & $43.688 \pm 1.420$ & 3 & 1 & $5.726 \pm 0.186$ & $83.5 \pm 7.9$ & $84.5 \pm 10.6$ & $81.0 \pm 10.1$ & $81.1 \pm 8.6$ & $76.2 \pm 7.0$ \\
\hline SJ102 & & -31.55989 & -68.66165 & 786 & $10 / 20 / 30$ & Chert & 26.3904 & 0.3487 & 1.3540 & $49.158 \pm 1.359$ & 5 & 1 & $5.877 \pm 0.162$ & $87.4 \pm 8.1$ & $88.5 \pm 11.0$ & $84.8 \pm 10.4$ & $85.0 \pm 8.9$ & $79.8 \pm 7.2$ \\
\hline SJ103 & & -31.55989 & -68.66146 & 783 & $18 / 25 / 30$ & $\begin{array}{l}\text { Chert with } \\
\text { limestone }\end{array}$ & 20.2383 & 0.3676 & 1.3540 & $28.668 \pm 1.067$ & 5 & 1 & $4.711 \pm 0.175$ & $69.9 \pm 6.7$ & $70.6 \pm 8.9$ & $67.8 \pm 8.5$ & $72.8 \pm 7.3$ & $64.1 \pm 6.0$ \\
\hline SJ104 & & -31.55951 & -68.66101 & 785 & $10 / 27 / 30$ & Chert & 24.2659 & 0.3475 & 1.3540 & $30.058 \pm 0.900$ & 2 & 1 & $3.895 \pm 0.117$ & $56.1 \pm 5.2$ & $56.8 \pm 7.0$ & $54.3 \pm 6.7$ & $54.3 \pm 5.7$ & $50.9 \pm 4.6$ \\
\hline SJ105 & & -31.55953 & -68.66101 & 777 & $10 / 20 / 25$ & $\begin{array}{l}\text { Limestone } \\
\text { with chert }\end{array}$ & 25.9175 & 0.3633 & 1.3540 & $32.268 \pm 0.811$ & 4 & 1 & $4.092 \pm 0.103$ & $60.3 \pm 5.5$ & $61.4 \pm 7.5$ & $58.9 \pm 7.2$ & $6.1 \pm 5.5$ & $55.2 \pm 4.9$ \\
\hline SJ142 & Q4 $4_{\ln }$ & -31.56222 & -68.66187 & 783 & $8 / 8 / 7$ & Chert & 27.2129 & 0.3411 & 1.3540 & $56.758 \pm 1.470$ & 3 & 1 & $6.437 \pm 0.167$ & $97.7 \pm 9.1$ & $98.3 \pm 12.2$ & $94.6 \pm 11.6$ & $94.8 \pm 9.9$ & $89.3 \pm 8.1$ \\
\hline SJ143 & & -31.56217 & -68.66185 & 786 & $10 / 8 / 7$ & Chert & 22.3433 & 0.3485 & 1.3540 & $64.538 \pm 3.598$ & 3 & 1 & $9.108 \pm 0.559$ & $139.4 \pm 16.4$ & $136.3 \pm 18.8$ & $131.1 \pm 17.9$ & $130.1 \pm 9.9$ & $89.3 \pm 8.1$ \\
\hline SJ144 & & -31.56218 & -68.66176 & 786 & $14 / 7 / 9$ & Chert & 24.2076 & 0.3570 & 1.3540 & $53.138 \pm 1.799$ & 3 & 1 & $7.090 \pm 0.240$ & $10.5 \pm 10.5$ & $108.7 \pm 13.7$ & $104.7 \pm 13.1$ & $104.7 \pm 11.2$ & $99.3 \pm 9.2$ \\
\hline SJ145 & & -31.56220 & -68.66174 & 796 & $10 / 8 / 5$ & Chert & 20.2153 & 0.3582 & 1.3540 & $39.848 \pm 2.255$ & 3 & 1 & $6.388 \pm 0.361$ & $94.5 \pm 10.0$ & $85.2 \pm 12.8$ & $91.6 \pm 12.2$ & $91.8 \pm 10.7$ & $86.3 \pm 9.0$ \\
\hline SJ146 & & -31.56221 & -68.66180 & 793 & $9 / 9 / 5$ & Chert & 21.3544 & 0.3559 & 1.3540 & $68.568 \pm 2.458$ & 3 & 1 & $10.340 \pm 0.371$ & $155.5 \pm 15.2$ & $153.1 \pm 19.6$ & $146.7 \pm 18.7$ & $146.5 \pm 15.9$ & $138.1 \pm 13.1$ \\
\hline SJ147 & & -31.56220 & -68.66185 & 795 & $12 / 9 / 6$ & Chert & 22.4149 & 0.3489 & 1.3540 & $49.368 \pm 1.365$ & 3 & 1 & $6.953 \pm 0.192$ & $104.0 \pm 9.7$ & $103.6 \pm 12.9$ & $99.9 \pm 12.4$ & $100.0 \pm 10.5$ & $94.7 \pm 8.6$ \\
\hline SJ137 & Q5 $\mathrm{ln}$ & -31.55652 & -68.66815 & 849 & $13 / 9 / 7$ & Chert & 24.8338 & 0.3427 & 1.3540 & $109.888 \pm 2.561$ & 3 & 1 & $13.720 \pm 0.320$ & $204.0 \pm 19.3$ & $198.4 \pm 25.1$ & $191.3 \pm 25.1$ & $190.9 \pm 20.3$ & $182.0 \pm 116.7$ \\
\hline SJ138 & & -31.55652 & -68.66817 & 842 & $12 / 9 / 7$ & & 26.6105 & 0.3481 & 1.354 & $92.698 \pm 13.026$ & 3 & 1 & $10.971 \pm 1.542$ & $162.3 \pm 27.9$ & $159.4 \pm 30.4$ & $152.7 \pm 29.0$ & $152.4 \pm 27.2$ & $144.0 \pm 24.5$ \\
\hline SJ140 & & -31.55631 & -68.66808 & 847 & $10 / 8 / 5$ & $\mathrm{Ch}$ & 1341 & 0.3695 & 1.3540 & $88.788 \pm 2.326$ & 3 & 1 & $14.045 \pm 0.368$ & $205.8 \pm 19.7$ & $200.2 \pm 25.4$ & $192.8 \pm 24.3$ & $192.3 \pm 20.6$ & $183.5 \pm 17.0$ \\
\hline SJ141 & & -31.55648 & -68.66833 & 846 & $11 / 6 / 5$ & Chert & 22.2781 & 0.3678 & 1.3540 & $109.888 \pm 2.681$ & 3 & 1 & $16.414 \pm 0.400$ & $242.9 \pm 23.3$ & $237.9 \pm 30.4$ & $227.3 \pm 28.9$ & $226.7 \pm 24.4$ & $214.6 \pm 19.9$ \\
\hline \multicolumn{19}{|l|}{$\angle A C A$} \\
\hline ArQ1-1 & $\mathrm{Q} 1_{\mathrm{c}}$ & -31.78764 & -68.57603 & 633 & $12 / 9.5 / 8$ & Quartz & 22.7842 & 0.3439 & 1.4140 & $3.351 \pm 0.008$ & 8 & 1 & $0.478 \pm 0.001$ & $7.9 \pm 0.7$ & $8.8 \pm 1.0$ & $8.4 \pm 1.0$ & $8.7 \pm 0.9$ & $7.9 \pm 0.7$ \\
\hline ARQ1-2 & & -31.78764 & -68.57603 & 633 & $11.5 / 10 / 5$ & Quartz & 25.8342 & 0.3412 & 1.4140 & $12.59 \pm 0.467$ & 5 & 1 & $1.571 \pm 0.058$ & $25.6 \pm 2.4$ & $27.5 \pm 3.4$ & $26.4 \pm 3.3$ & $26.7 \pm 2.8$ & $24.7 \pm 2.3$ \\
\hline ArQ1-4 & & -31.78764 & -68.57603 & 633 & $11 / 8.5 / 7$ & Quar & 25.2126 & 0.3569 & 1.4140 & $9.187 \pm 1.147$ & 7 & 1 & $1.229 \pm 0.153$ & $20.3 \pm 3.1$ & $22.2 \pm 3.8$ & $21.3 \pm 3.7$ & $21.6 \pm 3.5$ & $19.9 \pm 3.0$ \\
\hline ArQ1-5 & & -31.78764 & -68.57603 & 633 & 8.5 & Q & 25.4621 & 0.3366 & 1.4140 & $12.249 \pm 0.415$ & 5 & 1 & $1.530 \pm 0.052$ & $24.9 \pm 2.3$ & $26.9 \pm 3.3$ & $25.7 \pm 3.2$ & $26.1 \pm 2.7$ & $24.1 \pm 2.2$ \\
\hline ARQ2a-2 & Q2a $\mathrm{a}_{\mathrm{c}}$ & -31.78762 & -68.57555 & 641 & $8.5 / 7 / 4.5$ & Quartz & 26.1875 & 0.3226 & 1.4140 & $34.001 \pm 1.484$ & 4.5 & 1 & $3.958 \pm 0.173$ & $64.4 \pm 6.4$ & $66.0 \pm 8.4$ & $63.5 \pm 8.1$ & $63.7 \pm 7.0$ & $59.6 \pm 5.7$ \\
\hline ArQ2a-4 & & -31.78762 & -68.57555 & 641 & $11 / 6 / 3.5$ & Quartz & 25.1722 & 0.3454 & 1.4140 & $4.092 \pm 0.121$ & 3.5 & 1 & $0.531 \pm 0.016$ & $8.4 \pm 0.8$ & $9.4 \pm 1.1$ & $8.9 \pm 1.1$ & $9.2 \pm 1.0$ & $8.5 \pm 0.8$ \\
\hline ArQ2a-5 & & -31.78762 & -68.57555 & 641 & $6 / 5 / 3$ & Qua & 21.7400 & 0.3568 & 1.4140 & $0.768 \pm 1.296$ & 3 & 1 & $0.119 \pm 0.201$ & $1.9 \pm 3.2$ & $2.2 \pm 3.8$ & $2.0 \pm 3.4$ & $2.2 \pm 3.7$ & $2.0 \pm 3.4$ \\
\hline ARQ2b-3 & $\mathrm{Q} 2 \mathrm{~b}_{\mathrm{c}}$ & -31.78768 & -68.57538 & 638 & $7 / 4 / 3.6$ & $\mathrm{Qu}$ & 27.9179 & 0.3492 & 1.4140 & $48.241 \pm 0.916$ & 3.5 & 1 & $5.701 \pm 0.108$ & $92.9 \pm 8.5$ & $95.1 \pm 11.6$ & $91.3 \pm 11.1$ & $91.7 \pm 9.4$ & $85.6 \pm 7.6$ \\
\hline $\mathrm{ArQ} 2 \mathrm{~b}-4$ & & -31.78768 & -68.57538 & 638 & $9.5 / 7 / 5$ & Quartz & 21.6964 & 0.3528 & 1.4140 & $1.117 \pm 0.040$ & 5 & 1 & $0.172 \pm 0.006$ & $2.8 \pm 0.3$ & $3.3 \pm 0.4$ & $3.1 \pm 0.4$ & $3.3 \pm 0.3$ & $3.0 \pm 0.3$ \\
\hline ArQ2b-5 & & -31.78768 & -68.57538 & 638 & $12 / 7 / 3$ & Quartz & 24.1383 & 0.3512 & 1.4140 & $4.903 \pm 0.170$ & 3 & 1 & $0.674 \pm 0.023$ & $10.7 \pm 1.0$ & $12.0 \pm 1.5$ & $11.4 \pm 1.4$ & $11.7 \pm 1.2$ & $10.8 \pm 1.0$ \\
\hline ARQ3a-2 & Q3a $\mathrm{a}_{\mathrm{c}}$ & -31.78805 & -68.57573 & 641 & $8.5 / 7.8 / 4.3$ & Qua & 24.9512 & 0.3317 & 1.4140 & $17.161 \pm 0.403$ & 3 & 1 & $2.156 \pm 0.051$ & $34.4 \pm 3.1$ & $35.9 \pm 4.4$ & $34.5 \pm 4.2$ & $34.9 \pm 3.6$ & $32.5 \pm 2.9$ \\
\hline ARQ3a-3 & & -31.78805 & -68.57573 & 641 & $8.2 / 5.2 / 3$ & Quartzite & 27.8906 & 0.3556 & 1.4140 & $55.691 \pm 2.051$ & 3 & 1 & $6.709 \pm 0.247$ & $109.0 \pm 10.6$ & $109.5 \pm 13.9$ & $105.6 \pm 13.3$ & $105.7 \pm 11.4$ & $99.6 \pm 9.4$ \\
\hline ArQ3a-4 & & -31.78805 & -68.57573 & 641 & $10 / 9 / 5$ & Quartz & 26.7480 & 0.3599 & 1.4140 & $15.819 \pm 0.762$ & 5 & 1 & $2.011 \pm 0.097$ & $32.6 \pm 3.3$ & $34.3 \pm 4.4$ & $32.9 \pm 4.2$ & $33.4 \pm 3.7$ & $31.0 \pm 3.0$ \\
\hline ArQ3a-5 & & -31.78805 & -68.57573 & 641 & $8.5 / 3.5 / 4$ & Quartz & 22.6127 & 0.3501 & 1.4140 & $5.005 \pm 4.430$ & 4 & 1 & $0.732 \pm 0.648$ & $11.7 \pm 10.4$ & $13.1 \pm 11.8$ & $12.5 \pm 11.2$ & $12.8 \pm 11.5$ & $11.7 \pm 10.5$ \\
\hline
\end{tabular}




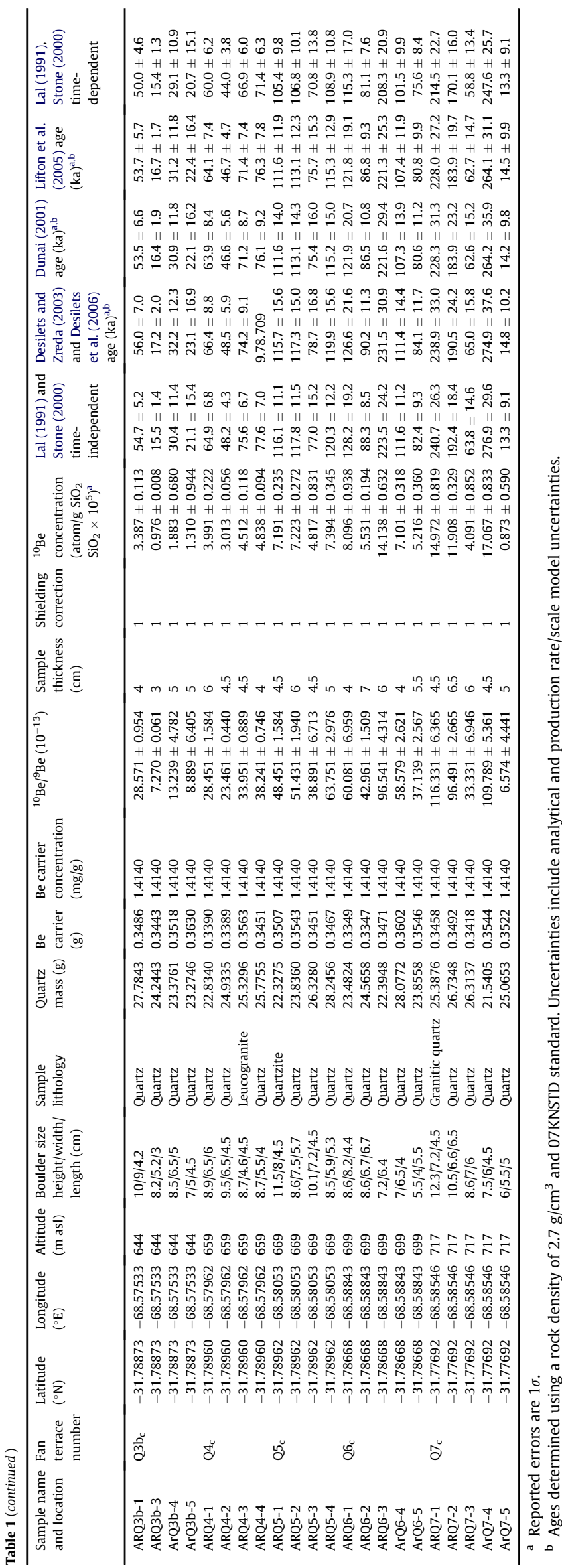

the 250-500 $\mu \mathrm{m}$ grain size fraction, which was then put through a series of acid leaches. Each sample was leached in $\mathrm{HCl}$ and $\mathrm{H}_{2} \mathrm{O}_{2}$ and HF, as outlined in Seong et al. (2009). Once pure, the obtained quartz was rinsed with de-ionized $\mathrm{H}_{2} \mathrm{O}$ and dried before being placed on $10 \mathrm{~mm}$ diameter stainless steel disks and held in place with silicone spray for measurement. The luminescence signals were measured using a Riso TL/OSL reader (model DA-20). Luminescence from the quartz grains was stimulated using an array of blue-light-emitting diodes (470 nm, $50 \mathrm{~mW} \mathrm{~cm} \mathrm{~cm}^{-2}$ ), filtered using a green long-pass GG-420 filter; detection was through a Hoya U-340 filter. All quartz aliquots were screened for feldspar contamination using infrared stimulation with infraredlight-emitting diodes $\left(870 \mathrm{~nm}, 150 \mathrm{~mW} \mathrm{~cm}^{-2}\right)$. All OSL signals were measured using a 52-mm-diameter photomultiplier tube (9235B). The equivalent dose (DE) measurements were determined on multiple aliquots using the single aliquot regenerative (SAR) method protocol developed by Walling et al. (2000). Growth curve data were fitted using linear and exponential trend curves. The DE value for every aliquot was examined using Riso Analysis $3.22 \mathrm{~b}$ software. Aliquots with poor recuperation $(>10 \%)$ were not used in the age calculations.

Equivalent doses of all aliquots were averaged for each sample then divided by the dose rate giving a mean age.

\section{Field location descriptions and sampling sites}

\subsection{Marquesado strath terrace complex}

The Marquesado field site is a small $\left(<1 \mathrm{~km}^{2}\right)$ strath terrace complex located on a shooting range within a military base. The ephemeral feeder channel exits the Sierra de Villicum range, a northeast-southwest trending range with peaks that reach $\sim 1000 \mathrm{~m}$ asl that rises westwards above the strath terrace surfaces, which are at an altitude of $\sim 800 \mathrm{~m}$ asl. Subsequent strath terrace deposits are traversed by a series of thrust faults that are roughly parallel to the range front. Four distinct fault scarps-back thrusts or bedding plane thrusts-are visible traversing the alluvial fan surface (Fig. 2).

The westernmost fault scarp cutting the Marquesado complex is $\sim 1 \mathrm{~m}$ high. There is no evidence for lateral displacement along the fault; uplift of the hanging wall resulted in the development of a sag pond on the footwall and caused the diversion of the small channel along the trace of the fault for $\sim 15 \mathrm{~m}$ to the northeast. Faults to the east were not closely investigated in the field.

Four abandoned strath terrace surfaces comprise the Marquesado complex $\left(\mathrm{Q} 2 \mathrm{~m}\right.$ through $\mathrm{Q} 5_{\mathrm{m}}$; Fig. 2). Dissection of the strath terrace surfaces increases with the relative age surface and the distance from the mountain front. Three fault scarps cut the strath terrace surfaces (Fig. 2). Although vertical offset of these scarps was not measured in the field, based on photographs and Google imagery, the youngest scarp appears to be significantly higher in relief than older scarps. The toe of these strath terrace deposits has experienced significant erosion due to the action of fluvial processes (Fig. 2).

Five 5-6 cm-long, chert and jasper cobbles were collected for ${ }^{10} \mathrm{Be}$ dating from the $\mathrm{Q} 3_{\mathrm{m}}$ surface on the hanging wall $\sim 20 \mathrm{~m}$ from the youngest fault scarp. The $\mathrm{Q}_{\mathrm{m}}$ surface has a moderately developed desert pavement and comprises many clasts with welldeveloped rock varnish (Fig. 2).

Most clasts comprise limestone and/or chert. Although small boulders ( $<30 \mathrm{~cm}$ long-axis) are present on the surface, due to limited access they could not be sampled. Instead, five cobbles were collected from the strath terrace surface. 
Table 2

Terrestrial cosmogenic nuclide depth profile, erosion rate data, and ${ }^{10} \mathrm{Be}$ measurement results.

\begin{tabular}{|c|c|c|c|c|c|c|c|c|c|c|c|c|c|}
\hline Surface & $\begin{array}{l}\text { Sample } \\
\text { name }\end{array}$ & $\begin{array}{l}\text { Depth or } \\
\text { sample } \\
\text { thickness } \\
(\mathrm{cm})\end{array}$ & Latitude $\left({ }^{\circ} \mathrm{N}\right)$ & $\begin{array}{l}\text { Longitude } \\
\left({ }^{\circ} \mathrm{E}\right)\end{array}$ & $\begin{array}{l}\text { Altitude } \\
\text { (m asl) }\end{array}$ & $\begin{array}{l}\text { Quartz } \\
\text { mass (g) }\end{array}$ & $\begin{array}{l}\text { Be } \\
\text { carrier } \\
\text { (g) }\end{array}$ & $\begin{array}{l}\text { Be carrier } \\
\text { concentration } \\
(\mathrm{mg} / \mathrm{g})\end{array}$ & $\begin{array}{l}{ }^{10} \mathrm{Be} /{ }^{9} \mathrm{Be} \\
\left(10^{-13}\right)\end{array}$ & $\begin{array}{l}{ }^{10} \mathrm{Be} \text { concentration } \\
\left(\text { atom } / \mathrm{g} \mathrm{SiO}_{2}\right. \\
\left.\mathrm{SiO}_{2} \times 10^{5}\right)^{\mathrm{a}}\end{array}$ & $\begin{array}{l}\text { Inheritance }{ }^{\mathrm{b}} \\
\left(10^{4} \text { atoms/g) }\right.\end{array}$ & $\begin{array}{l}\text { Erosion rate }{ }^{\mathrm{a}, \mathrm{b}, \mathrm{c}} \\
(\mathrm{m} / \mathrm{Myr})\end{array}$ & $\mathrm{Age}^{\mathrm{b}, \mathrm{c}}(\mathrm{ka})$ \\
\hline \multicolumn{14}{|l|}{ LOMA NEGRA } \\
\hline \multirow{5}{*}{$\mathrm{Q} 2_{\ln }$} & SJ123 & 0 & -31.55973 & -68.66340 & 790 & 21.4489 & 0.3418 & 1.3540 & $3.167 \pm 0.131$ & $4.566 \pm 0.189$ & $11.97 .5-11.9$ & $5.90 .3-7.1$ & $45.737 .8-53.4$ \\
\hline & SJ125 & $70 \pm 5$ & & & & 14.7596 & 0.3673 & 1.3540 & $0.985 \pm 0.041$ & $2.217 \pm 0.093$ & & & \\
\hline & SJ126 & $100 \pm 5$ & & & & 24.3983 & 0.3577 & 1.3540 & $3.307 \pm 0.122$ & $4.386 \pm 0.162$ & & & \\
\hline & SJ127 & $130 \pm 5$ & & & & 24.4183 & 0.3447 & 1.3540 & $1.469 \pm 0.061$ & $1.876 \pm 0.078$ & & & \\
\hline & SJ128 & $170 \pm 5$ & & & & 20.4067 & 0.3602 & 1.3540 & $4.281 \pm 0.202$ & $6.836 \pm 0.322$ & & & \\
\hline \multirow[t]{5}{*}{ Q3 $\ln$} & SJ129 & 0 & -31.55796 & -68.66228 & 785 & 22.5963 & 0.3606 & 1.3540 & $2.630 \pm 0.166$ & $3.797 \pm 0.240$ & $11.98 .9-11.9$ & $2.30 .4-8.0$ & $39.634 .9-48.5$ \\
\hline & SJ131 & $70 \pm 5$ & & & & 20.0247 & 0.3524 & 1.3540 & $1.842 \pm 0.092$ & $2.933 \pm 0.146$ & & & \\
\hline & SJ132 & $100 \pm 5$ & & & & 22.4443 & 0.3660 & 1.3540 & $1.698 \pm 0.063$ & $2.505 \pm 0.093$ & & & \\
\hline & SJ133 & $130 \pm 5$ & & & & 15.3392 & 0.3516 & 1.3540 & $0.925 \pm 0.042$ & $1.918 \pm 0.087$ & & & \\
\hline & SJ134 & $170 \pm 5$ & & & & 21.9308 & 0.3420 & 1.3540 & $4.099 \pm 0.156$ & $5.783 \pm 0.221$ & & & \\
\hline Catchment & SJ106 & 2.5 & -31.56339 & -68.68080 & 1155 & 23.9029 & 0.3652 & 1.3540 & $5.748 \pm 0.798$ & $7.945 \pm 1.103$ & - & $7.6 \pm 1.3$ & $80.9 \pm 13.4$ \\
\hline Ridge bedrock & SJ107 & 1 & -31.56339 & -68.68080 & 1146 & 24.6541 & 0.3450 & 1.3540 & $8.135 \pm 0.238$ & $10.030 \pm 0.030$ & & $5.9 \pm 0.5$ & $102.7 \pm 9.4$ \\
\hline Catchment & SJ113 & 4 & -31.56127 & -68.67296 & 842 & 21.8382 & 0.3662 & 1.3540 & $3.031 \pm 0.068$ & $4.598 \pm 0.103$ & - & $10.9 \pm 0.9$ & - \\
\hline Hillslope & SJ114 & 2 & -31.56305 & -68.67401 & 852 & 22.0878 & 0.3563 & 1.3540 & $1.539 \pm 0.255$ & $2.246 \pm 0.372$ & & $24.4 \pm 4.7$ & \\
\hline Active & SJ115 & - & -31.56353 & -68.67355 & 854 & 23.1551 & 0.3373 & 1.3540 & $0.870 \pm 0.048$ & $1.146 \pm 0.063$ & - & $48.8 \pm 7.0$ & - \\
\hline Channel & SJ116 & & -31.55949 & -68.67100 & 819 & 23.8687 & 0.3601 & 1.3540 & $0.292 \pm 0.013$ & $0.399 \pm 0.0180$ & & $140.0 \pm 19.6$ & \\
\hline \multicolumn{14}{|l|}{ LA CARPINTERÍA } \\
\hline \multirow[t]{5}{*}{ Q5 c } & Q540-50 & $45 \pm 5$ & -31.789617 & -68.580533 & 669 & 24.0815 & 0.3436 & 1.414 & $2.438 \pm 0.117$ & $2.286 \pm 0.158$ & $9.88 .4-11.0$ & $2.70 .2-3.3$ & $\mathbf{8 0 . 1} 66.6-99.2$ \\
\hline & Q590-120 & $105 \pm 5$ & & & & 28.1202 & 0.3396 & 1.414 & $2.166 \pm 0.089$ & $2.414 \pm 0.101$ & & & \\
\hline & Q5120-140 & $130 \pm 5$ & & & & 19.5534 & 0.3494 & 1.414 & $0.970 \pm 0.041$ & $1.637 \pm 0.070$ & & & \\
\hline & Q5140-150 & $145 \pm 5$ & & & & 22.4758 & 0.3636 & 1.414 & $1.035 \pm 0.026$ & $1.581 \pm 0.040$ & & & \\
\hline & Q5240-250 & $245 \pm 5$ & & & & 30.1266 & 0.3477 & 1.414 & $1.096 \pm 0.046$ & $1.195 \pm 0.050$ & & & \\
\hline
\end{tabular}

- Indicates no data/not applicable.

a Reported errors are $1 \sigma$.

${ }^{\mathrm{b}}$ For depth profiles, Bayesian most probable values (bold) and $2 \sigma$ upper and lower age range as determined by Monte Carlo simulation.

${ }^{c}$ Production rate for active channel samples as determined through GIS and MATLAB analysis (as discussed in text) is $9.31 \pm 1.23 \mathrm{~g} \mathrm{~cm}^{-1} \mathrm{yr}^{-1}$. 
Table 3

\begin{tabular}{|c|c|c|c|c|c|c|c|c|c|c|c|c|c|}
\hline Sample name & Surface & $\begin{array}{l}\text { Average } \\
\text { pitting } \\
\text { depth }(\mathrm{cm})\end{array}$ & Pavement & $\begin{array}{l}\text { Desert } \\
\text { varnish }\end{array}$ & $\begin{array}{l}\text { Time-dependent } \\
\text { age }^{\mathrm{a}}\end{array}$ & $\begin{array}{l}\text { Age (inheritance } \\
\text { removed) })^{\mathrm{a}, \mathrm{b}}\end{array}$ & $\begin{array}{l}\text { Maximum } \\
\text { age }\end{array}$ & $\begin{array}{l}\text { Minimum } \\
\text { age }\end{array}$ & $\begin{array}{l}2 \sigma \text { mean } \\
\text { age }^{c}\end{array}$ & PDF age ${ }^{c}$ & $M_{S W D}{ }^{c}$ & $\begin{array}{l}\text { Depth profile } \\
\text { age }^{\mathrm{d}}\end{array}$ & OSL age \\
\hline \multicolumn{14}{|l|}{ MARQUESADO } \\
\hline SJ135B & $\mathrm{Q} 3 \mathrm{~m}$ & - & WD & HV & $14.6 \pm 1.3$ & 0 & 16.0 & 13.3 & $17.2 \pm 3.1$ & $88.0 \pm 4.5$ & $15.9 \pm 1.2$ & - & - \\
\hline SJ135C & & & & & $15.2 \pm 1.4$ & 0 & 16.6 & 13.8 & & & & & \\
\hline SJ135F & & & & & $16.2 \pm 2.4$ & 0 & 18.6 & 13.8 & & & & & \\
\hline SJ135A & & & & & $17.5 \pm 1.6$ & $0.8 \pm 0.6$ & 19.1 & 15.9 & & & & & \\
\hline SJ135E & & & & & $22.5 \pm 2.0$ & $6.1 \pm 0.9$ & 24.5 & 20.4 & & & & & \\
\hline SJ135D & & & & & $88.7 \pm 8.3$ & $72.5 \pm 7.0$ & 97.0 & 80.4 & & & & & \\
\hline \multicolumn{14}{|l|}{ LOMA NEGRA } \\
\hline SJ109 & $\mathrm{Q} 1_{\mathrm{ln}}$ & 0 & NP & LV & $3.1 \pm 0.3$ & 0 & 3.4 & 2.8 & $24.3 \pm 2.6$ & $31.0 \pm 2.8$ & - & - & - \\
\hline SJ112 & & & & & $11.0 \pm 1.0$ & 0 & 12.1 & 10.0 & & & & & \\
\hline SJ111 & & & & & $22.5 \pm 2.3$ & $5.9 \pm 1.4$ & 24.8 & 20.2 & & & & & \\
\hline SJ110 & & & & & $26.1 \pm 2.3$ & $10.0 \pm 1.1$ & 28.4 & 23.8 & & & & & \\
\hline SJ108 & & & & & $42.6 \pm 4.2$ & $28.5 \pm 3.2$ & 46.8 & 38.5 & & & & & \\
\hline SJ117 & $\mathrm{Q}_{\ln }$ & 4.3 & MD & HV & $47.1 \pm 4.4$ & $32.5 \pm 3.4$ & 51.5 & 42.6 & $48.0 \pm 4.8$ & $47.5 \pm 2.0$ & $48.0 \pm 1.6$ & $45.737 .8-53.4$ & - \\
\hline SJ118 & & & & & $47.2 \pm 4.2$ & $32.6 \pm 3.0$ & 51.4 & 43.0 & & & & & \\
\hline SJ120 & & & & & $47.3 \pm 4.2$ & $32.6 \pm 3.0$ & 51.5 & 43.1 & & & & & \\
\hline SJ119e & & & & & $47.7 \pm 17.5$ & $32.9 \pm 17.8$ & 65.1 & 30.2 & & & & & \\
\hline SJ121 & & & & & $50.7 \pm 4.6$ & $35.9 \pm 3.4$ & 55.3 & 46.2 & & & & & \\
\hline SJ122 & & & & & $73.7 \pm 6.6$ & $57.8 \pm 5.2$ & 80.3 & 67.2 & & & & & \\
\hline SJ104 & $\mathrm{Q}_{\ln }$ & 4.8 & MD & HV & $50.9 \pm 4.6$ & $36.1 \pm 3.4$ & 55.5 & 46.3 & $65.2 \pm 12.7$ & $70.0 \pm 20.0$ & $56.7 \pm 6.7$ & 39.6 34.9-48.5 & - \\
\hline SJ105 & & & & & $55.2 \pm 4.9$ & $39.2 \pm 3.6$ & 60.1 & 50.3 & & & & & \\
\hline SJ103 & & & & & $64.1 \pm 6.0$ & $47.0 \pm 4.7$ & 70.1 & 58.1 & & & & & \\
\hline SJ101 & & & & & $76.2 \pm 7.0$ & $60.5 \pm 5.8$ & 83.2 & 69.2 & & & & & \\
\hline SJ102 & & & & & $79.8 \pm 7.2$ & $63.6 \pm 5.9$ & 87.0 & 27.6 & & & & & \\
\hline SJ145 & Q4 $4_{\text {ln }}$ & 4.6 & WD & HV & $86.3 \pm 9.0$ & $69.9 \pm 7.8$ & 95.2 & 77.3 & $105.3 \pm 20.9$ & $93.0 \pm 30.0$ & $92.4 \pm 5.8$ & - & - \\
\hline SJ142 & & & & & $89.3 \pm 8.1$ & $72.5 \pm 6.6$ & 97.4 & 81.3 & & & & & \\
\hline SJ147 & & & & & $94.7 \pm 8.6$ & $78.3 \pm 7.2$ & 103.3 & 86.1 & & & & & \\
\hline SJ144 & & & & & $99.3 \pm 9.2$ & $82.9 \pm 7.9$ & 108.5 & 90.0 & & & & & \\
\hline SJ143 & & & & & $89.3 \pm 8.1$ & $108.5 \pm 12.3$ & 137.4 & 110.7 & & & & & \\
\hline SJ146 & & & & & $138.1 \pm 13.1$ & $122.0 \pm 11.8$ & 151.2 & 125.0 & & & & & \\
\hline SJ138 & Q5 $\ln$ & 5.4 & WD & HV & $144.0 \pm 24.5$ & $127.7 \pm 23.6$ & 168.4 & 119.5 & $181.0 \pm 28.9$ & $175.0 \pm 50.0$ & $193.3 \pm 18.4$ & - & - \\
\hline SJ137 & & & & & $182.0 \pm 116.7$ & $165.4 \pm 15.2$ & 198.7 & 165.3 & & & & & \\
\hline SJ140 & & & & & $183.5 \pm 17.0$ & $167.3 \pm 15.5$ & 200.4 & 166.5 & & & & & \\
\hline SJ141 & & & & & $214.6 \pm 19.9$ & $198.1 \pm 18.4$ & 234.4 & 194.7 & & & & & \\
\hline \multicolumn{14}{|l|}{ LA CARPINTERÍA } \\
\hline ARQ1-1 & $\mathrm{Q} 1_{\mathrm{c}}$ & - & NP & LV & $7.9 \pm 0.7$ & 0 & 8.6 & 7.3 & $22.9 \pm 2.9$ & $24.5 \pm 5.0$ & $22.9 \pm 2.6$ & - & - \\
\hline ArQ1-4 & & & & & $19.9 \pm 3.0$ & $4.3 \pm 2.7$ & 22.9 & 16.9 & & & & & \\
\hline ArQ1-5 & & & & & $24.1 \pm 2.2$ & $8.9 \pm 1.1$ & 26.3 & 21.9 & & & & & \\
\hline ARQ1-2 & & & & & $24.7 \pm 2.3$ & $9.6 \pm 1.3$ & 27.0 & 22.4 & & & & & \\
\hline ArQ2a-5 ${ }^{\mathrm{e}}$ & ${\mathrm{Q} 2 \mathrm{a}_{\mathrm{c}}}$ & - & NP & NV & $2.0 \pm 3.4$ & 0 & 5.4 & 0.0 & - & $6.0 \pm 1.0$ & - & - & \\
\hline ArQ2a-4 & & & & & $8.5 \pm 0.8$ & 0 & 9.2 & 7.7 & & & & & \\
\hline ARQ2a-2 & & & & & $59.6 \pm 5.7$ & $44.1 \pm 4.6$ & 65.3 & 53.8 & & & & & $4.6 \pm 0.9$ \\
\hline $\operatorname{ArQ2b-4}$ & $\mathrm{Q}^{2} \mathrm{~b}_{\mathrm{c}}$ & - & NP & NV & $3.0 \pm 0.3$ & 0 & 93.1 & 78.0 & - & $80.5 \pm 10.0$ & - & - & $4.9 \pm 0.5$ \\
\hline $\operatorname{ArQ2b-5}$ & & & & & $10.8 \pm 1.0$ & 0 & 11.7 & 9.8 & & & & & \\
\hline$A R Q 2 b-3$ & & & & & $85.6 \pm 7.6$ & $70.5 \pm 6.3$ & 93.1 & 78.0 & & & & & \\
\hline ArQ3a-5e & Q3a $\mathrm{a}_{\mathrm{c}}$ & - & MD & LV & $11.7 \pm 10.5$ & 0 & 22.2 & 1.3 & $25.1 \pm 11.6$ & $18.0 \pm 20.5$ & - & - & - \\
\hline ArQ3a-4 & & & & & $31.0 \pm 3.0$ & $16.5 \pm 2.1$ & 34.0 & 27.9 & & & & & \\
\hline ARQ3a-2 & & & & & $32.5 \pm 2.9$ & $18.4 \pm 1.8$ & 35.4 & 29.7 & & & & & \\
\hline ARQ3a-3 & & & & & $99.6 \pm 9.4$ & $85.3 \pm 8.3$ & 109.0 & 90.2 & & & & & \\
\hline ARQ3b-3 & Q3b $\mathrm{b}_{\mathrm{c}}$ & - & MD & LV & $15.4 \pm 1.3$ & 0 & 16.7 & 14.1 & $28.8 \pm 15.2$ & $25.5 \pm 17.0$ & $21.7 \pm 6.9$ & - & - \\
\hline ArQ3b-5 & & & & & $20.7 \pm 15.1$ & $5.4 \pm 15.6$ & 35.8 & 5.6 & & & & & \\
\hline ArQ3b-4 & & & & & $29.1 \pm 10.9$ & $14.5 \pm 11.0$ & 39.9 & 18.2 & & & & & \\
\hline ARQ3b-1 ${ }^{\mathrm{e}}$ & & & & & $50.0 \pm 4.6$ & $36.3 \pm 3.5$ & 54.5 & 45.4 & & & & & \\
\hline
\end{tabular}




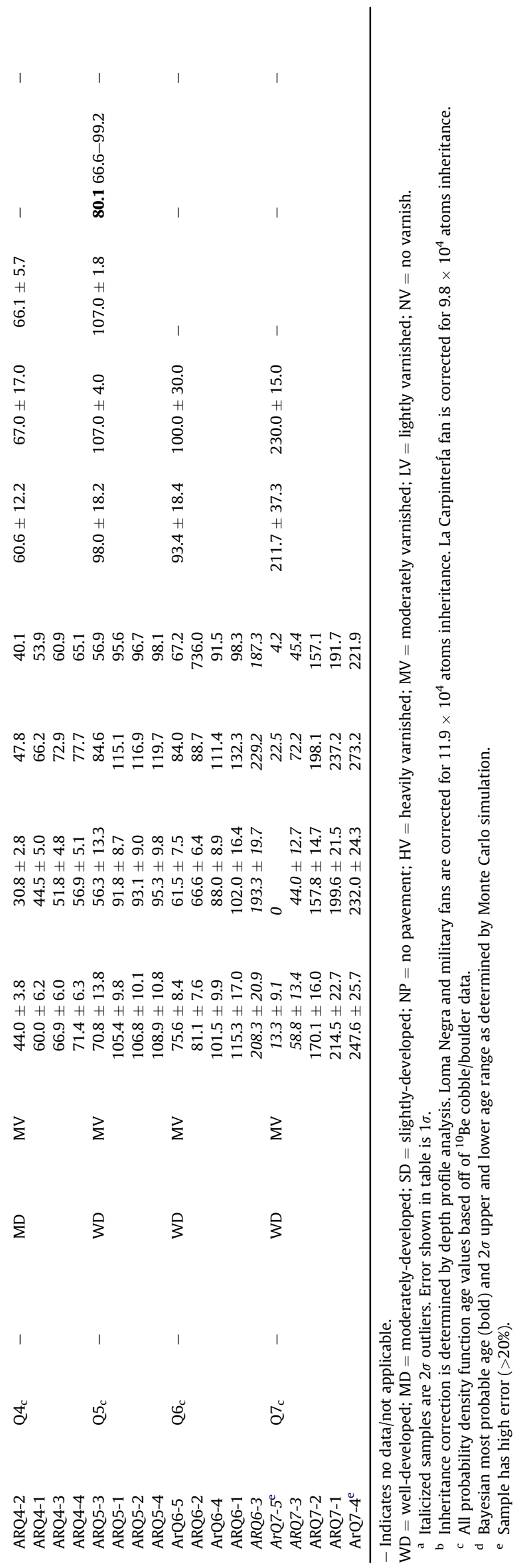

\subsection{Loma Negra alluvial fan complex}

The Loma Negra alluvial fan complex is located $\sim 10 \mathrm{~km}$ west of San Juan (Fig. 1). The active feeder channel now drains to the northeast, away from the majority of the alluvial fan deposits, and follows the modern day mountain front (Fig. 3). The highest peak above the basin is $\sim 1650 \mathrm{~m}$ asl, $\sim 800 \mathrm{~m}$ higher than the highest alluvial fan surface. The alluvial fan deposits span an area of $\sim 3.5 \mathrm{~km}^{2}$. The active channel $\left(\mathrm{Q} 1_{\mathrm{ln}}\right)$ experiences only ephemeral flow. The northernmost portion of this alluvial fan from modern channel to toe has been extensively mined for gravels and sands by the nearby Loma Negra Cement Factory, and has been used as a dumping ground for residual materials (Fig. 4 - waste pile). This modification is constrained to a relatively small area, which is not used in this study. However, there is a well-established road and footpath on the $\mathrm{Q}_{\ln }$ and $\mathrm{Q} 3_{\ln }$ surfaces. Samples were collected in areas where the desert pavement on these surfaces appeared to be undisturbed, and with the exception of some Q2 $\ln$ samples, were collected far ( $>50 \mathrm{~m}$ ) from both the road and footpath.

The Loma Negra alluvial fan complex comprises four surfaces. As determined from both field and Google Earth imagery a series of roughly north-south trending faults cut the Q2 In surface, and elsewhere separate the $\mathrm{Q}_{2}$ In and $\mathrm{Q}_{\ln }$ and Q4 $4_{\ln }$ surfaces (Fig. 3). The easternmost fault has produced a $3 \mathrm{~m}$-high scarp, but the height varies due to the fact that it separates the $\mathrm{Q} 2$ In surface from both the Q3 In surface and the oldest, highest Q4 In surface. This fault scarp is roughly parallel to the range front but faces uphill, trapping sediment on the footwall block. On this alluvial fan, the fault scarp separates the older $\mathrm{Q}_{\text {In }}$ and $\mathrm{Q} 3{ }_{\text {In }}$ surfaces entirely from the main body of the fan $(\mathrm{Q} 2 \mathrm{In})$. Similar to the Marquesado alluvial fan complex, the toe of this alluvial fan has experienced significant erosion due to the action of fluvial processes. Remnants of the old, high Q5 In surface are isolated to two large hills, the northernmost of which has been mined for gravel and sand. The tops of these deposits are $\sim 60 \mathrm{~m}$ higher than the surrounding ( $\mathrm{Q} 2 \mathrm{ln}$ ) surface.

The Q2 ${ }_{1 n}-\mathrm{Q} 5$ In surfaces all display the development of desert pavements to varying degrees (Fig. 4), as well as dark rock varnish on chert clasts, and the presence of larger boulders seems to diminish from the younger terraces to the older terraces. The high Q5 In surface is covered by a well-developed pavement of limestone and chert clasts, the largest of which are $\sim 10 \mathrm{~cm}$ long axis. Based on the presence of concentrated gravels throughout the subsurface soils as seen in depth profiles for the Q2 $\ln$ and Q $3_{\text {In }}$ surfaces, these desert pavements have likely formed primarily as deflationary surfaces through winnowing and fluvial processes.

At the Loma Negra alluvial fan complex (Fig. 3), we sampled the largest clasts available that were suitable for dating on each surface, which averaged a long axis of $\sim 12 \mathrm{~cm}$. Clasts containing quartz lithologies on the alluvial fan surfaces were restricted to boulders and cobbles which originated as limestone containing chert veins; most clasts today are composed mainly of dark, well-varnished chert, but some still contain significant amounts of limestone. The limestone and/or chert boulders sampled in the active channel, which are representative of the overall boulder population, have an average long axis of $58 \mathrm{~cm}$. Boulders or cobbles were sampled for ${ }^{10} \mathrm{Be}$ dating from each major alluvial fan surface (Q2 $\left.{ }_{\ln }-\mathrm{Q} 5_{\ln }\right)$ (Fig. 4). Four to six cobbles or boulders were sampled for each surface. Most samples were collected $>50 \mathrm{~m}$ from fault scarps (Fig. 3), although some boulders sampled from the Q2In surface (SJ117, SJ118, and SJ119) were sampled $\sim 20 \mathrm{~m}$ of the fault scarp and may show influence of inheritance due to denudation of the fault scarp. Boulders of mixed chert and limestone are present on the Q2 1 surface (41.2 cm average length) as well as on the Q3 3 surface $(34 \mathrm{~cm}$ average). On the $\mathrm{Q}^{3}$ n surface, the largest clasts are composed dominantly of chert. Sampled clasts on the Q4 $\ln$ surface also had 

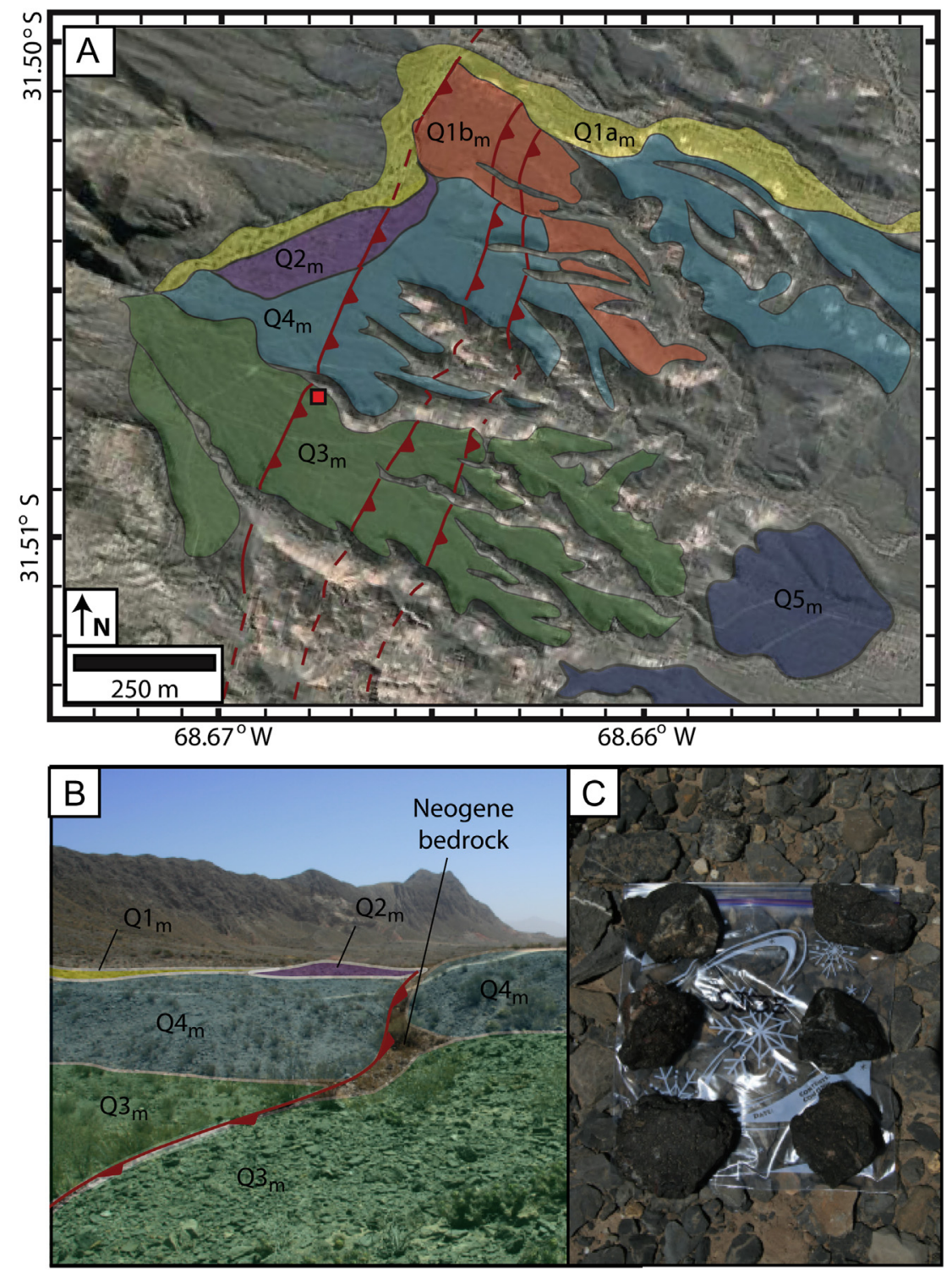

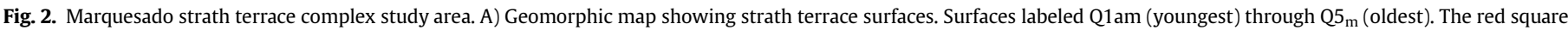

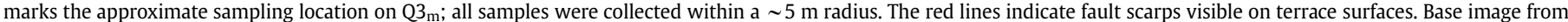

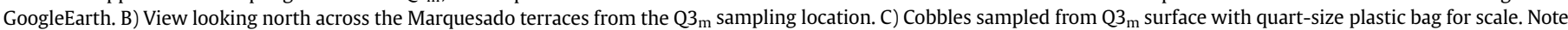

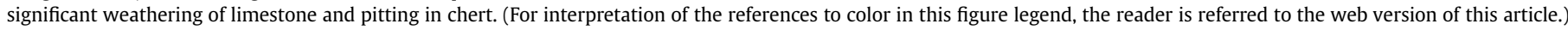

average lengths of $\sim 12 \mathrm{~cm}$. On the oldest ( $\left.\mathrm{Q} 5_{\ln }\right)$ surface, boulders were absent and only chert cobbles, $\sim 10 \mathrm{~cm}$ long, were present; these were sampled. Due to the quartz-rich nature of the Loma Negra boulders, only $\sim 250-500 \mathrm{~g}$ of material was collected to obtain sufficient quartz for ${ }^{10} \mathrm{Be}$ dating.

Depth profile samples were collected on the $\mathrm{Q}_{2}$ In and $\mathrm{Q}_{3}$ In surfaces of the Loma Negra alluvial fan complex. The pits required to collect these samples were dug using a backhoe to a depth of $\sim 190 \mathrm{~cm}$, and samples of chert and quartz pebbles $(3-5 \mathrm{~cm}$ long) totaling $\sim 500 \mathrm{~g}$ were taken at each interval of $\sim 30 \mathrm{~cm}$.

Bedrock erosion rate samples were collected from the ridge above the feeder channel of the Loma Negra alluvial fan complex. These samples were collected from two different bedrock exposures at the top of a ridge at $\sim 1150 \mathrm{~m}$ asl. Sample SJ106 was collected from a low ( $\sim 45 \mathrm{~cm}$ height) bedrock exposure that crops out for a distance of $\sim 1 \mathrm{~m}$. SJ107 was collected from a similar but much longer outcrop $(\sim 4 \mathrm{~m})$. Both of these samples were collected from limestone rock containing beds of heavily-varnished chert (Fig. 4 - Bedrock).

Two hillslope erosion rate samples were collected from the northwestern edge of the Loma Negra alluvial fan feeder channel (Fig. 3G - SJ113 and SJ114) from high angle $\left(\sim 35^{\circ}\right)$ talus deposits. A collection comprising chert pebbles $\sim 5-10 \mathrm{~cm}$ long axis was collected weighing 250-500 g. Two catchment-wide erosion rate samples were collected directly from the Loma Negra fan feeder channel (Fig. 3H - SJ115 and SJ116). Sediment totaling $\sim 1 \mathrm{~kg}$ was collected from active channel sand deposits.

\subsection{Carpintería strath terrace complex}

The Carpintería complex is part of a large bajada that stretches for $\sim 25 \mathrm{~km}$ to the south of the Loma Negra alluvial fan complex, and may be better described as a sequence of strath terraces incised into a rising complex of basin deposits; the deposits are 

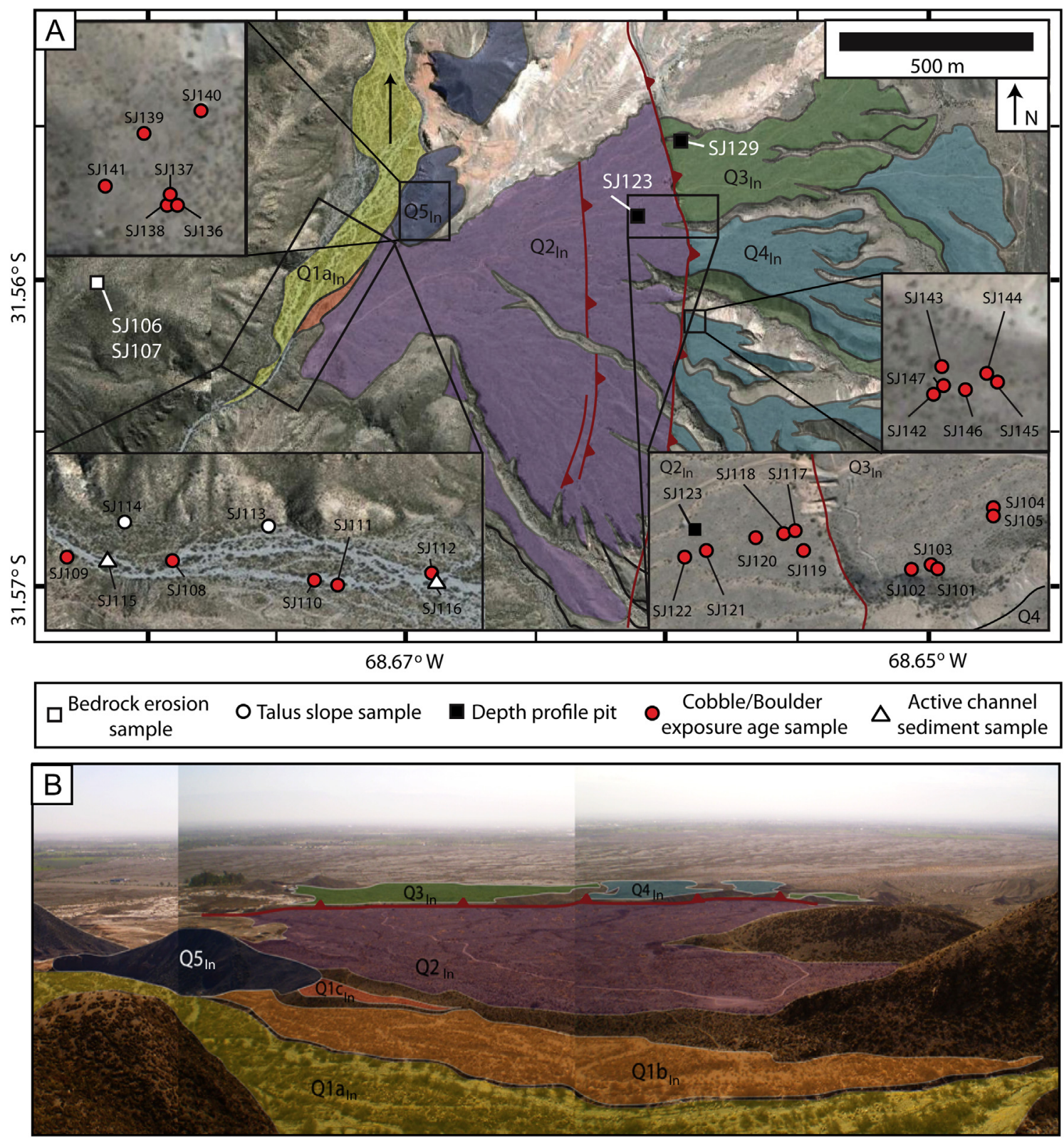

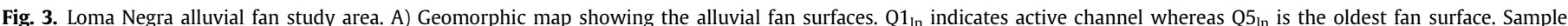

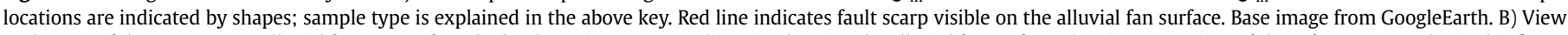

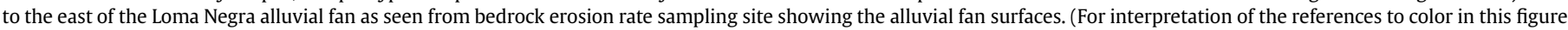
legend, the reader is referred to the web version of this article.)

located much farther from the range front $(\sim 2.5 \mathrm{~km})$ than both the Marquesado strath terraces and Loma Negra alluvial fan surfaces and are $>2 \mathrm{~m}$ in thickness, but these deposits lack the characteristic alluvial fan shape as they are confined within an incised canyon and are situated above a bedrock strath surface. These deposits are likely to have been more greatly affected by fluvial processes.

The Carpintería complex deposits investigated in this study span an area of $\sim 5.5 \mathrm{~km}^{2}$ and are interrupted by large fault scarps which have altered the distribution of feeder channels, and isolated these alluvial fan surfaces from the mountain range front. The range is oriented approximately north-south with peaks reaching $\sim 2000 \mathrm{~m}$ asl. The modern channels $\left(\mathrm{Q} 1_{\mathrm{c}}\right)$ have been 'bottlenecked' through a bedrock exposure uplifted through fault motion (Fig. 5) and carry only ephemeral flow.
Clasts in the active channel (Q1 $)$ are primarily rounded cobbles, pebbles, and sand (Fig. 6). The top of the $\mathrm{Q}_{2} \mathrm{~b}_{\mathrm{c}}$ surface is $\sim 2 \mathrm{~m}$ higher than the $\mathrm{Q} 1_{\mathrm{c}}$ surface at the $\mathrm{Q} 1_{\mathrm{c}}$ and $\mathrm{Q} 2_{\mathrm{c}}$ sampling sites (Fig. 5B). The Q2 c surfaces comprise primarily cobbles and pebbles, and desert pavement has begun to form on the surface of $\mathrm{Q}^{2} \mathrm{~b}_{\mathrm{c}}$. At the $\mathrm{Q}^{3} \mathrm{a}_{\mathrm{c}}$ surface, $\sim 3 \mathrm{~m}$ above the $\mathrm{Q} 2 \mathrm{~b}_{\mathrm{c}}$ surface, pedogenic development is evident in the presence of a $\sim 30 \mathrm{~cm}$ thick layer of gypsum. Chert and limestone cobbles and pebbles on this surface have been visibly weathered. The $\mathrm{Q}^{3} \mathrm{~b}_{\mathrm{c}}$ surface is not significantly higher than that of the $\mathrm{Q} 3 \mathrm{a}_{\mathrm{c}}$ surface, but displays a well-developed desert pavement. The $\mathrm{Q}_{\mathrm{c}}$ surface, $\sim 2-3 \mathrm{~m}$ above the $\mathrm{Q} 3 \mathrm{~b}_{\mathrm{c}}$ surface, also has a well-developed desert pavement as well as a developing soil containing gypsum.

The Q5 ${ }_{c}$ surface is $\sim 5-6 \mathrm{~m}$ higher than the $\mathrm{Q} 4_{\mathrm{c}}$ surface. $\mathrm{Q} 5_{\mathrm{c}}$ is characterized by highly fractured and angular clasts and an 


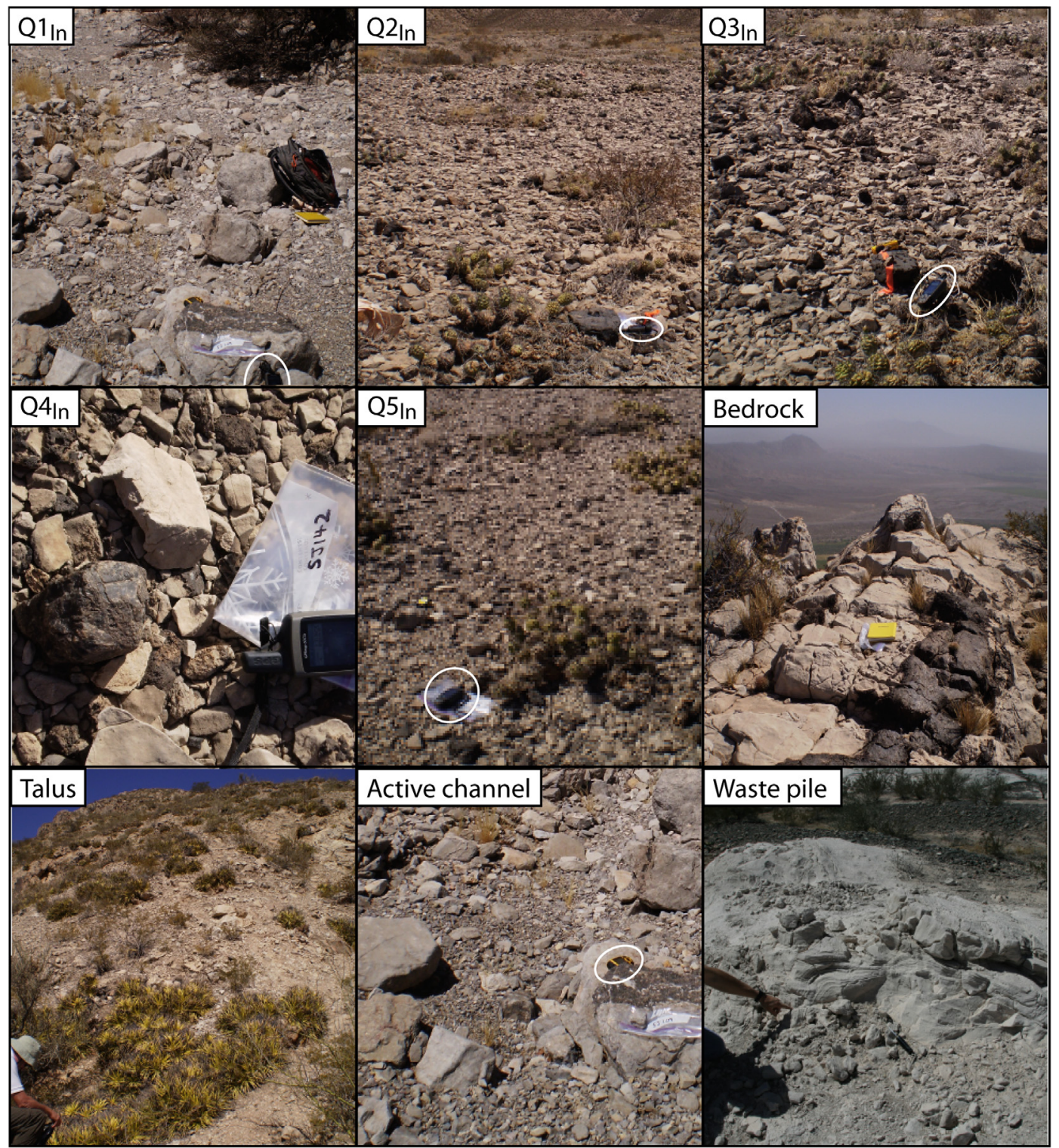

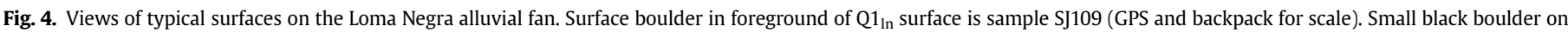

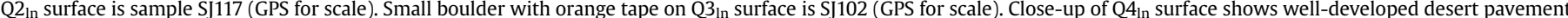

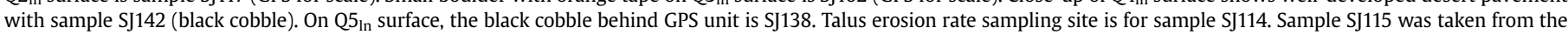
active channel site. The waste deposits show striated, fine-grained stratigraphy.

abundance of quartz pebbles in a well-developed desert pavement. $\mathrm{Q6}_{\mathrm{c}}$ rests $\sim 4-5 \mathrm{~m}$ above $\mathrm{Q} 5_{\mathrm{c}}$ and has a similarly well-developed pavement. Clasts on the $\mathrm{Q}_{\mathrm{c}}$ surface are primarily chert and quartz; chert pebbles display deep weathering whereas quartz clasts are rounded, suggesting that they are second generation clasts. The $\mathrm{Q}_{c}$ surface is only found on the northern side of the main drainage. This terrace is high ( $\sim 80 \mathrm{~m}$ above $\mathrm{Q} 1_{\mathrm{c}}$ ), deformed, and dissected.

The thick deposits of the older strath terraces allowed for exposures of the underlying soil stratigraphy. Soil profiles are described for the $\mathrm{Q6}_{\mathrm{c}}$ and $\mathrm{Q7}_{\mathrm{c}}$ terraces. Gypsum is common throughout the $\mathrm{Q}_{\mathrm{c}}$ soil profile, while the $\mathrm{Q6}_{\mathrm{c}}$ profile is characterized by calcium sulfate cementation in the A and Byk1 horizons with calcium carbonate cementation beginning in the Byk1 horizon and continuing into the Byk2. Gypsum is also present in the $\mathrm{Q6}_{\mathrm{c}}$ Byk1 horizon (Fig. 7).

Boulders were not present on the surfaces of the Carpintería alluvial fan complex (Figs. 5 and 6). Five large, quartz-rich cobbles (granitic quartz and quartzite) were collected from each surface $(\mathrm{Q} 2 \mathrm{c}-\mathrm{Q7}$ ) . A backhoe was used to dig a depth profile pit to a depth of $\sim 245 \mathrm{~cm}$ on the $\mathrm{Q}_{\mathrm{c}}$ surface. Six samples were collected at $\sim 50 \mathrm{~cm}$ intervals and $\sim 250-500 \mathrm{~g}$ of quartz-rich pebbles were collected per interval. Only pebbles $\sim 2-5 \mathrm{~cm}$ long were collected. Notable sedimentary and pedogenic features within the pit section were recorded.

Two OSL (San Juan 1 and 2) samples were collected from a deposit of silty sand with small pebbles $\sim 5 \mathrm{~m}$ from one-another in an exposed section within the Q2 ${ }_{c}$ surface of the Carpintería alluvial fan complex (Fig. 8).

\section{Results}

\section{1. ${ }^{10}$ Be clast ages and erosion rate results}

${ }^{10} \mathrm{Be}$ clast ages are listed in Table 1 and plotted in Fig. 9. No correction was made for erosion rate. However, we examine 


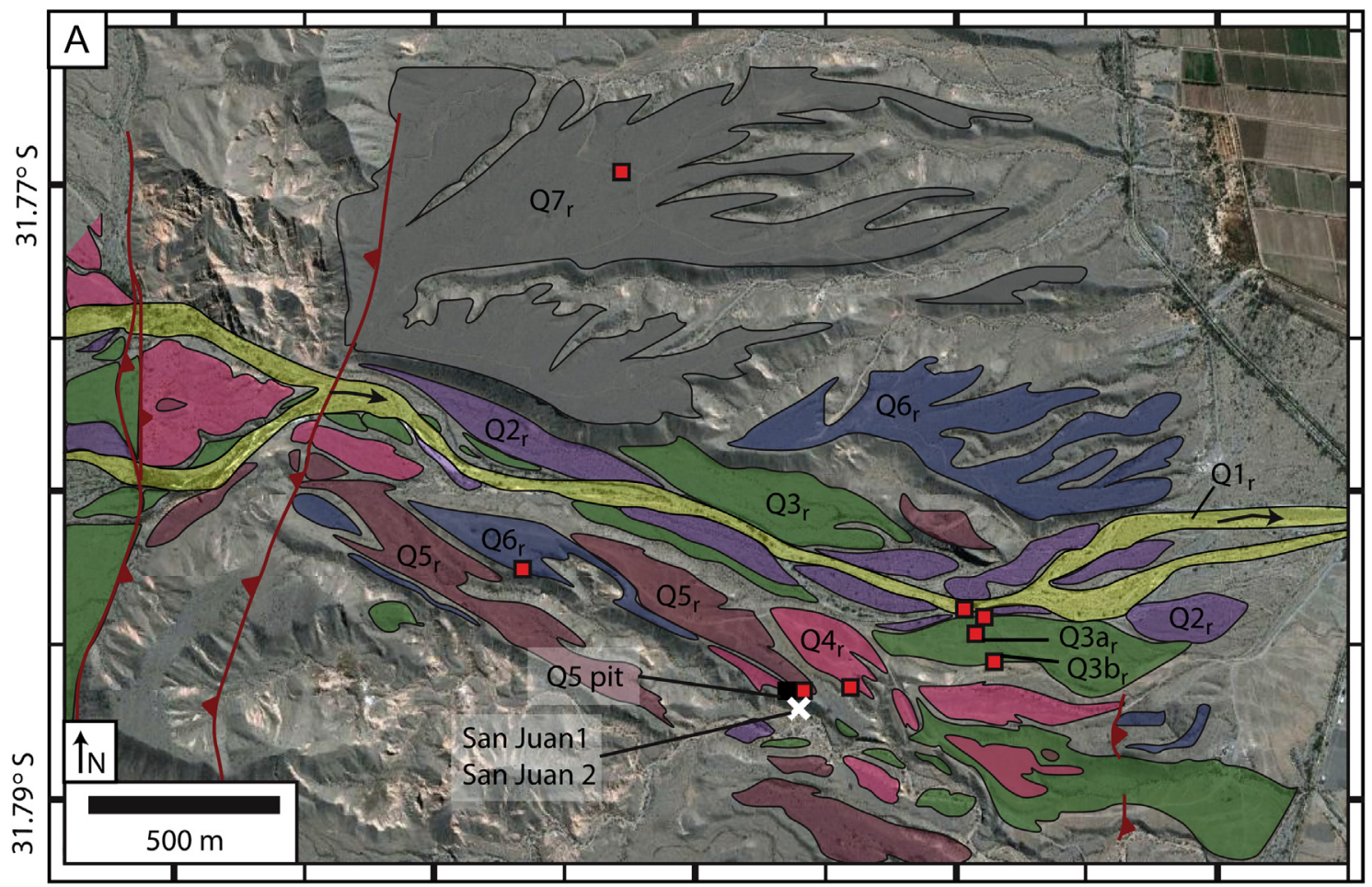
$68.60^{\circ} \mathrm{W}$
$68.58^{\circ} \mathrm{W}$
$68.56^{\circ} \mathrm{W}$

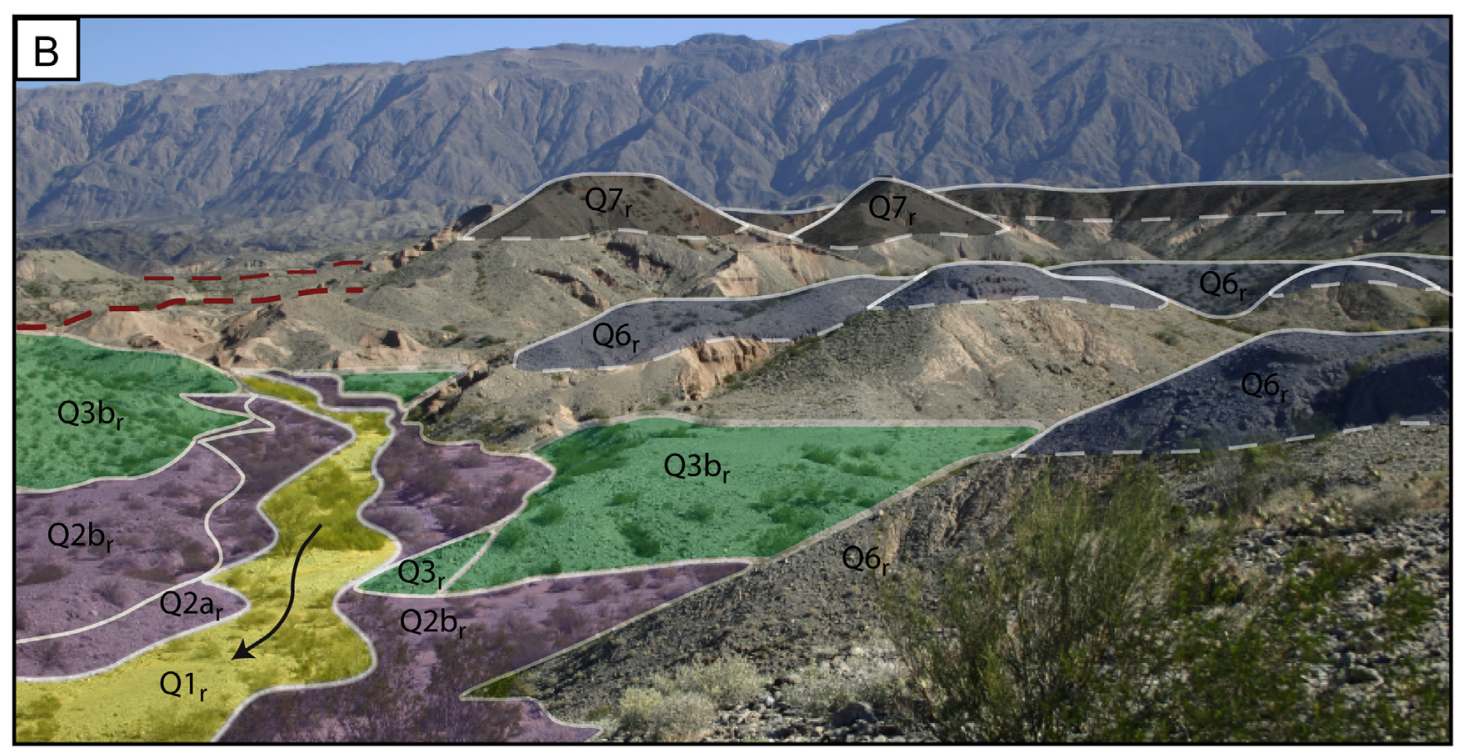

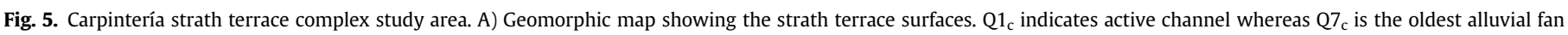

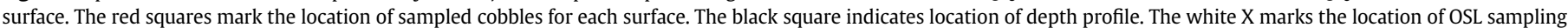

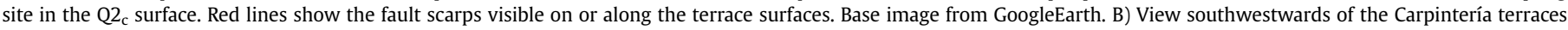
from the northern $\mathrm{Q}_{\mathrm{c}}$ surface. (For interpretation of the references to color in this figure legend, the reader is referred to the web version of this article.)

potential error in ${ }^{10} \mathrm{Be}$ concentration due to erosion rate by recalculating clast ages with reference erosion rates, and present the resulting percentage error between the original (erosion rate $=0 \mathrm{~m} \mathrm{Ma}^{-1}$ ) clast ages and the clast ages recalculated with reference erosion rates.

We consider a variety of evidence to determine our reference erosion rates: 1 ) The presence of clasts with ages as old as $\sim 200 \mathrm{ka}$ suggests that the surface erosion rate must be relatively low, and additionally, the preserved rounded clast forms of chert boulders and cobbles also supports the view that erosion rates are low; 2)
${ }^{10} \mathrm{Be}$ bedrock erosion rates determined through CRONUS calculation of samples SJ106 and SJ107 provide a mean bedrock erosion rate of $6.8 \pm 1.3 \mathrm{~m} / \mathrm{Ma}$ ) Analyses of pebbles in ${ }^{10} \mathrm{Be}$ depth profiles (discussed below) suggests that on both the $\mathrm{Q}_{2}$ nn and $\mathrm{Q}_{\text {In }}$ surfaces of the Loma Negra alluvial fan complex, the average rate of erosion is $5.7 \pm 0.9 \mathrm{~m} \mathrm{Ma}^{-1}$-depth profile analysis on the Carpintería $\mathrm{Q}_{\mathrm{c}}$ surface suggests a mean erosion rate of $\left.3.8 \pm 2.0 \mathrm{~m} \mathrm{Ma}^{-1} ; 4\right)$ Following the method of Lal (1991), we calculate a rough estimate of the mean erosion rate from the ${ }^{10} \mathrm{Be}$ exposure ages of the three oldest clasts $\left(\mathrm{Q} 7-1_{\mathrm{c}}, \mathrm{Q} 7-2_{\mathrm{c}}\right.$, and $\mathrm{Q7-4}$ ) as $2.8 \pm 0.6 \mathrm{~m} / \mathrm{Ma}$. 


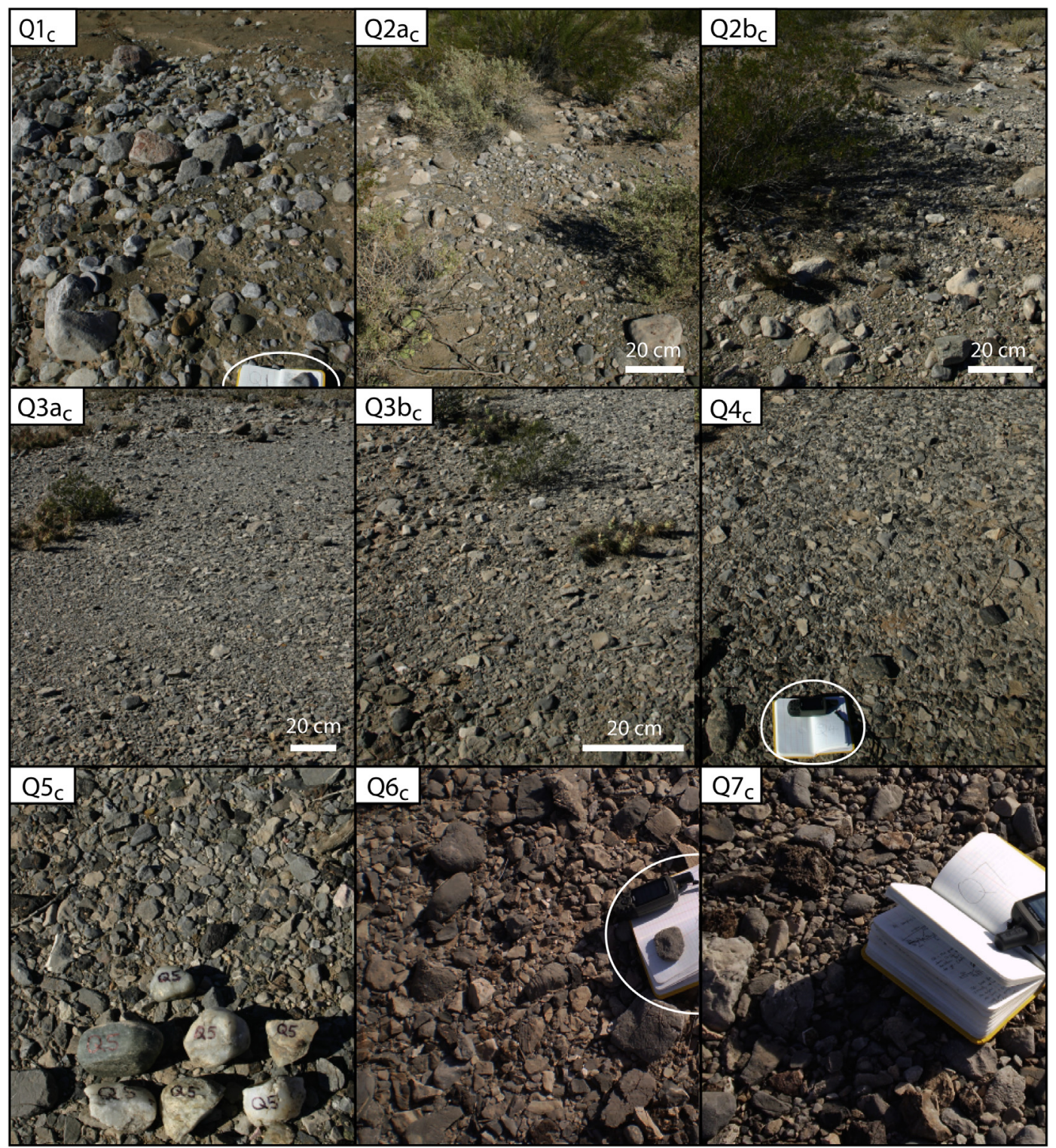

Fig. 6. Carpintería strath terraces, note field notebook for scale on $\mathrm{Q} 4_{c}, \mathrm{Q} 6_{c}$, and $\mathrm{Q} 7_{\mathrm{c}}$ surfaces. $\mathrm{Q} 5_{\mathrm{c}}$ surface shows the cobbles that were sampled for TCN dating.

All of these erosion rate values are very similar and are lower than the mean global ${ }^{10} \mathrm{Be}$-determined arid region bedrock erosion rates examined by Portenga and Bierman (2011), suggesting that they are reasonable erosion rate values for this hyper-arid region. Hillslope erosion rate and basin-wide erosion rate of the Loma Negra source basin are $17.7 \pm 4.7 \mathrm{~m} \mathrm{Ma}^{-1}$ and $94.4 \pm 19.6 \mathrm{~m} \mathrm{Ma}^{-1}$, respectively, which also agree with or are lower than the global rates determined by Portenga and Bierman (2011). These hillslope and catchment-wide erosion rates are not considered for our reference erosion rates because they take into account erosive processes which are not active on an in-situ clast or bedrock surface and would thus be highly inaccurate.

We provide quantitatively-defined bedrock or clast erosion rates ranging from $\sim 2 \mathrm{~m} \mathrm{Ma}^{-1}$ to $\sim 8 \mathrm{~m} \mathrm{Ma}^{-1}$. The reference erosion rates we chose for our recalculations are $1 \mathrm{~m} / \mathrm{Ma}, 3 \mathrm{~m} / \mathrm{Ma}$, and $8 \mathrm{~m} /$ $\mathrm{Ma}$. If the true erosion rate was $1 \mathrm{~m} \mathrm{Ma}^{-1}$, by using the assumption of $0 \mathrm{~m} \mathrm{Ma}^{-1}$ erosion rate we would be underestimating the age of a 10 ka sample by $\sim 1 \%, 50$ ka by $\sim 5 \%$, and 100 ka by $\sim 8 \%$. At $3 \mathrm{~m} \mathrm{Ma}^{-1}$ erosion rate, assuming $0 \mathrm{~m} \mathrm{Ma}^{-1}$ causes underestimations at $10 \mathrm{ka}$ of $\sim 3 \%, 50 \mathrm{ka}$ of $\sim 14 \%$, and $100 \mathrm{ka}$ of $\sim 21 \%$.
Using an erosion rate of $8 \mathrm{~m} \mathrm{Ma}^{-1}$ we find that calculated ages underestimate actual ages at $10 \mathrm{ka}$ by $\sim 8 \%, 50 \mathrm{ka}$ by $\sim 30 \%$, and at 100 ka would underestimate the age by $\sim 45 \%$.

Because we use an assumed erosion rate of $0 \mathrm{~m} \mathrm{Ma}^{-1}$ in our age calculations, the ages we report in Table 1 must be considered minimum ages. Our use of the Lal (1991)/Stone (2000) timedependent scaling scheme also produces the youngest ages as compared to all other scaling schemes. By using the scaling schemes of Lal (1991)/Stone (2000) time-constant, Desilets et al. (2006), Dunai (2001), and Lifton et al. (2005), the scaling schemes increase sample ages by a maximum of $11.5 \%, 10.3 \%, 5.9 \%$, and $8.1 \%$, respectively, for all time periods.

\section{2. ${ }^{10}$ Be surface ages and statistical analysis}

There has been much debate regarding how best to interpret TCN boulder/cobble data to determine the most accurate age of a geomorphic surface. Although prior studies aimed exclusively toward discussion and modeling of the interpretation of TCN boulder/ cobble exposure ages have primarily focused on moraines (cf. 

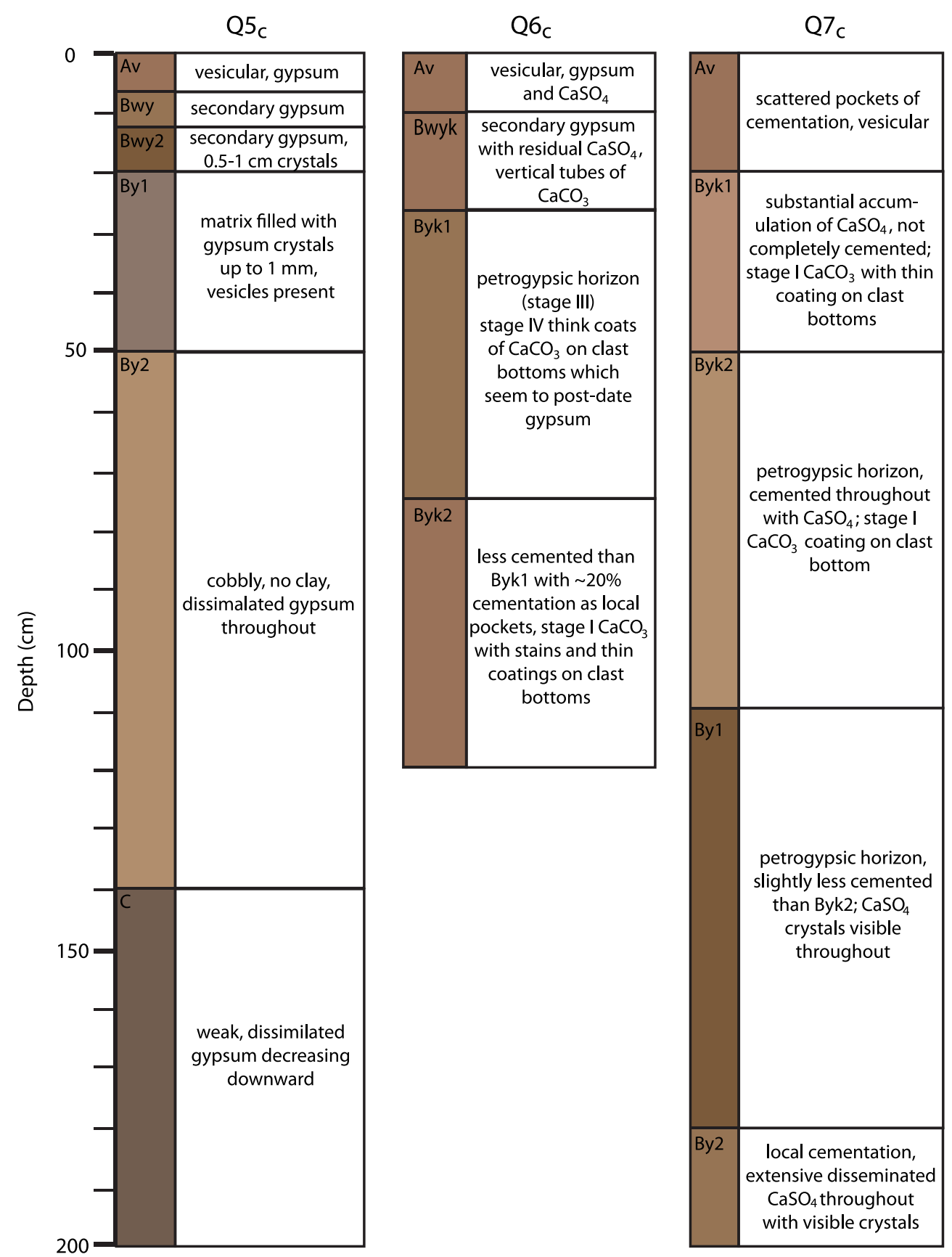

Fig. 7. Soil profiles for the $\mathrm{Q} 5_{\mathrm{c}}, \mathrm{Q} 6_{\mathrm{c}}$ and $\mathrm{Q} 7_{\mathrm{c}}$ surfaces for the Carpintería strath terrace complex.

Hallet and Putkonen, 1993; Putkonen and Swanson, 2003; Applegate et al., 2010, 2012), many sources of potential inaccuracies in TCN boulder/cobble dating also apply to alluvial fan and fluvial terrace surfaces and deposits (Hetzel et al., 2002; González et al., 2006; Schmidt et al., 2011). TCN boulder/cobble ages may overestimate the actual exposure age of a surface due to predepositional inheritance, or underestimate it due to shielding or extreme erosion of the sampled boulders/cobbles. We therefore examine our data with a variety of methods to attempt to explain potential anomalies and determine an accurate surface age from the boulder/cobble data.

We define the age of a surface by examining the ${ }^{10} \mathrm{Be}$ clast ages and apply $2 \sigma$ outlier, probability density function (PDF), and mean square weighted deviation (MSWD) tests; the results for these tests can be seen in Table 3 .
${ }^{10} \mathrm{Be}$ clast ages for each surface show considerable variation; however, clast ages from a given surface typically have few $2 \sigma$ outliers. AMS measurement results report external error at $1 \sigma$ values; for $2 \sigma$ mean age we apply the $2 \sigma$ error and remove all samples that are outliers from the dataset. Once these outliers are removed we take the mean age of the remaining sample cluster as the $2 \sigma$ mean age for that surface (Fig. 9). Due to the requisite tighter clustering of the remaining, non-outlier sample ages, a $1 \sigma$ error reported on the $2 \sigma$ age may be anomalously low, approaching analytical levels. External uncertainties realistically account for $\sim 10 \%$ error; we thus report $2 \sigma$ mean sample age with $10 \%$ error as surface ages except in cases where $1 \sigma$ is greater than $10 \%$ of the sample age $\left(\mathrm{Q}_{2}\right.$ n and $\left.\mathrm{Q}_{\mathrm{c}}\right)$. For these surfaces we use the greater value of error to reflect the contribution of geologic error to the surface age. We also plot the ${ }^{10} \mathrm{Be}$ clast ages of each surface as a PDF 


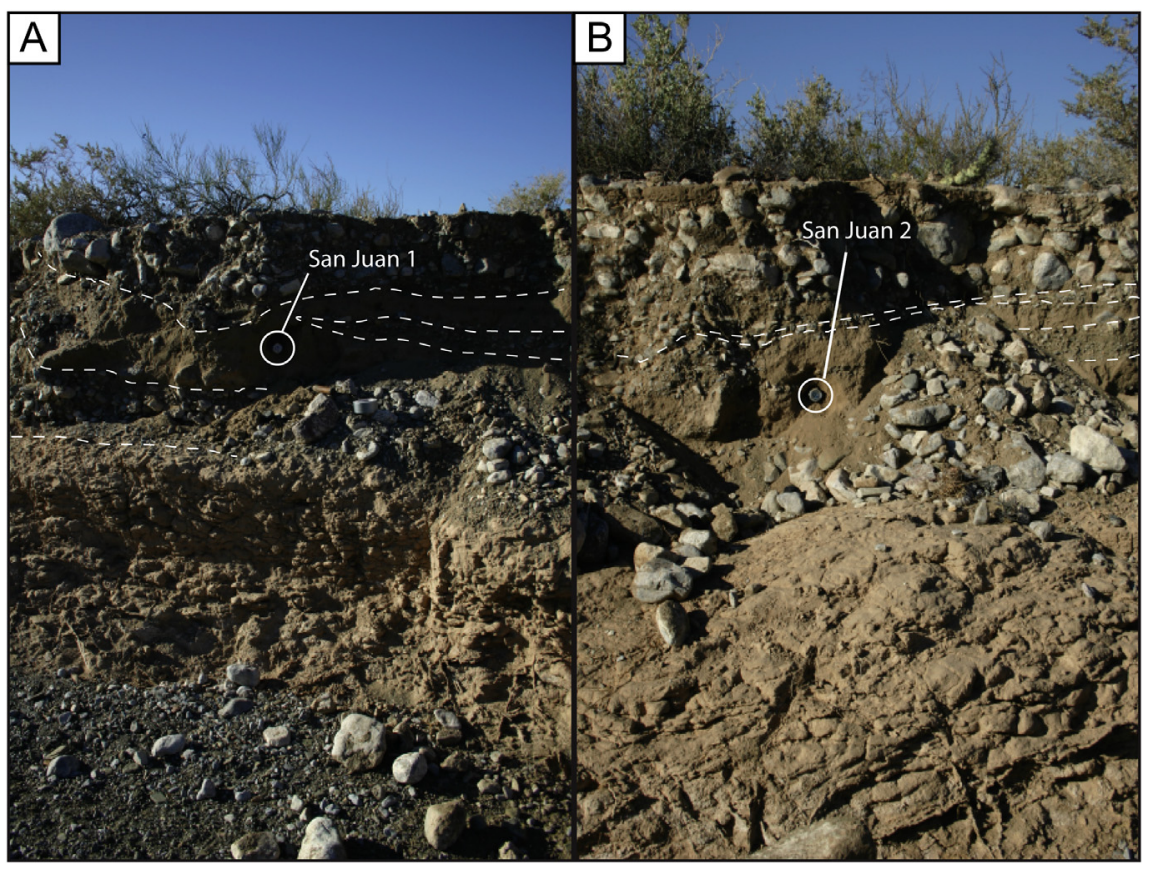

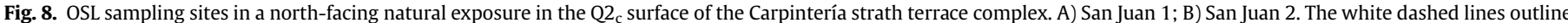
bedding contacts. San Juan 2 is $\sim 5$ m west of San Juan 1 .

in order to determine most likely age based on the range of age results (Fig. 10).

Additionally, we apply the MSWD method of Wendt and Carl (1991) to our sample sets as a way of determining the usefulness of the clast ages as an isochron for surface age; failed MSWD values
$\left(R^{2}<1\right)$ suggest that data scatter is due to more than just analytical uncertainties and potential geologic error must be carefully considered (Powell et al., 2002). Although some surfaces did not pass this statistical test $\left(\mathrm{Q} 1_{\mathrm{ln}}, \mathrm{Q} 2 \mathrm{a}_{\mathrm{c}}, \mathrm{Q} 2 \mathrm{~b}_{\mathrm{c}}, \mathrm{Q} 3 \mathrm{a}_{\mathrm{c}}, \mathrm{Q} 6_{\mathrm{c}}, \mathrm{Q} 7_{\mathrm{c}}\right)$, most do. MSWD mean ages for the Marquesado alluvial fan complex pass

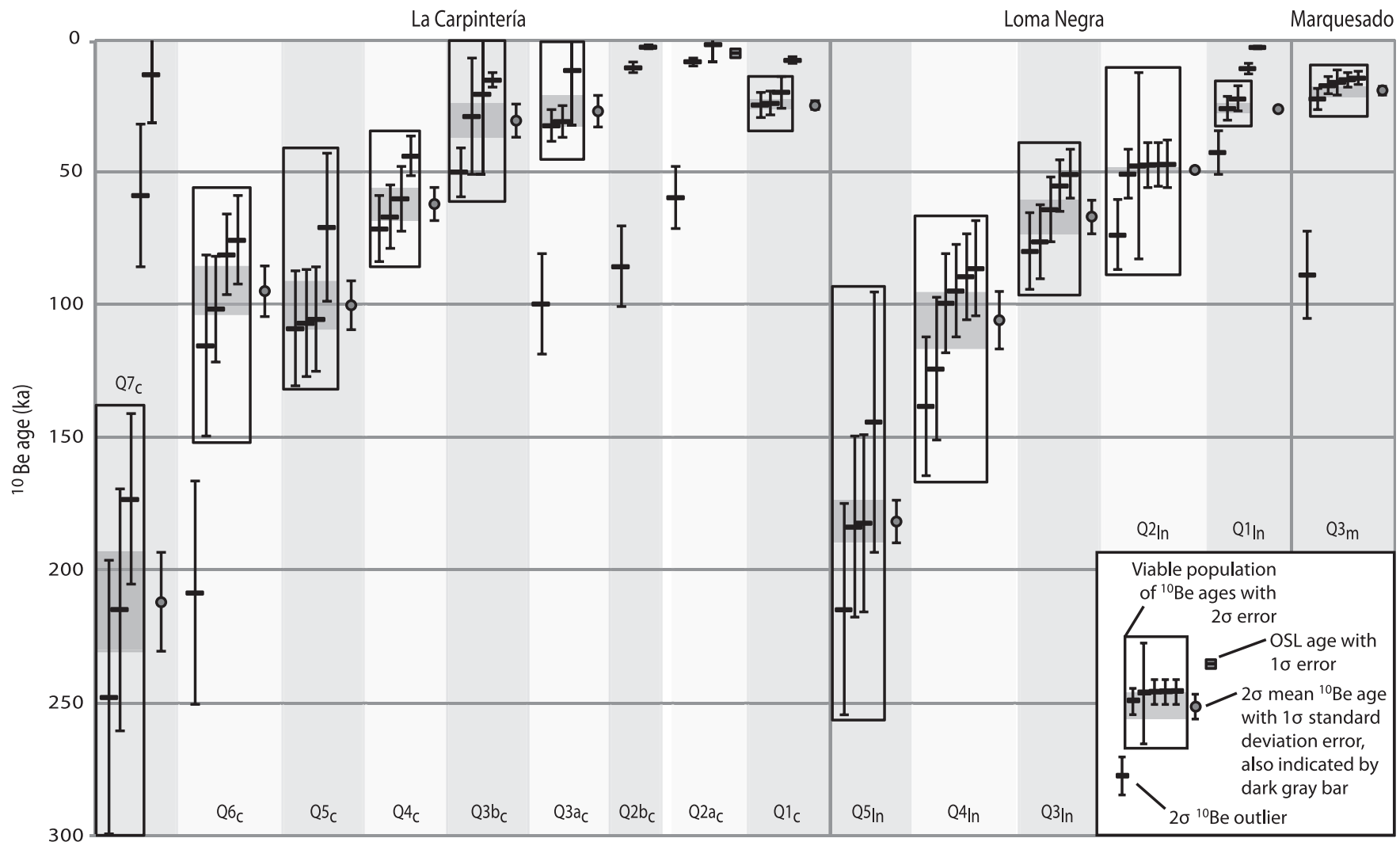

Relative age (arranged by alluvial fan surface)

Fig. 9. Plots of ${ }^{10} \mathrm{Be}$ ages for the Marquesado, Loma Negra, and Carpintería alluvial fan and strath terraces arranged by surface age. 

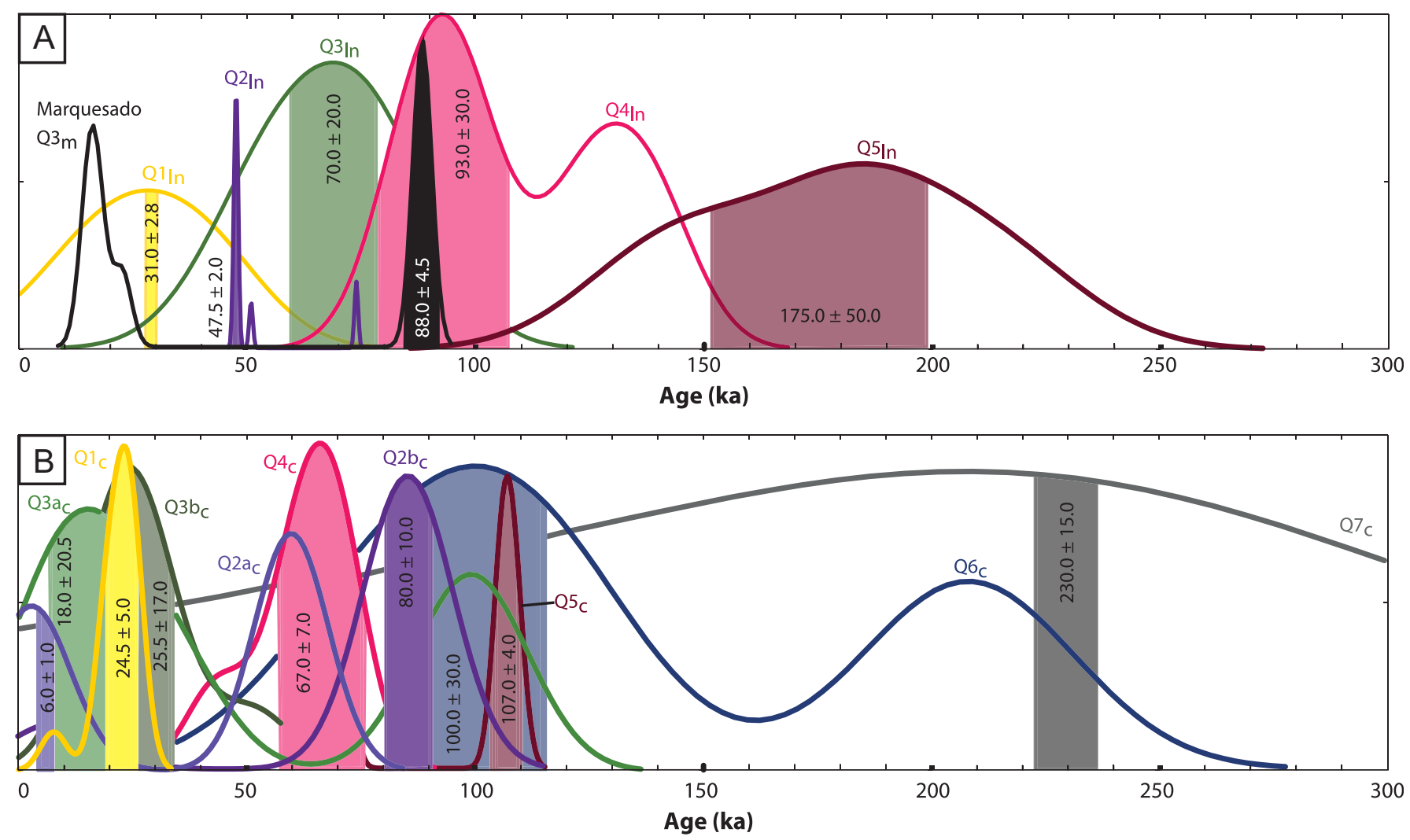

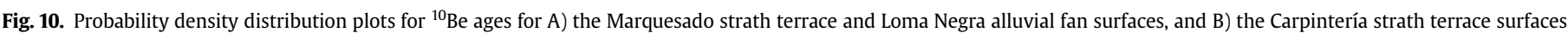

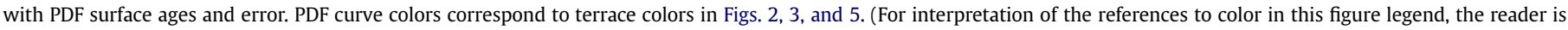
referred to the web version of this article.)

MSWD statistical analysis with an age of $15.9 \pm 1.2 \mathrm{ka}(1 \sigma$ error). The MSWD mean ages for the Loma Negra Q2 $2_{\ln }, \mathrm{Q} 3_{\ln }, \mathrm{Q} 4_{\ln }$, and Q $5_{\ln }$ surfaces are $48.0 \pm 1.6 \mathrm{ka}, 56.7 \pm 6.7 \mathrm{ka}, 92.4 \pm 5.7 \mathrm{ka}$, and $193.3 \pm 18.4$ ka, respectively. Results from Carpintería alluvial fan complex are less coherent, but the $\mathrm{Q} 1_{c}, \mathrm{Q} 3 \mathrm{~b}_{\mathrm{c}}, \mathrm{Q} 4_{c}$, and $\mathrm{Q} 5_{\mathrm{c}}$ surfaces pass MSWD analysis with mean ages $22.9 \pm 2.6 \mathrm{ka}, 21.7 \pm 6.9 \mathrm{ka}$, $66.1 \pm 5.7 \mathrm{ka}$, and $107.0 \pm 1.8 \mathrm{ka}$, respectively (Table 3).

MSWD analysis suggests that for surfaces $\mathrm{Q} 1_{\mathrm{ln}}, \mathrm{Q} 2 \mathrm{a}_{\mathrm{c}}, \mathrm{Q} 2 \mathrm{~b}_{\mathrm{c}}, \mathrm{Q} 3 \mathrm{a}_{\mathrm{c}}$, $\mathrm{Q6}_{\mathrm{c}}$, and $\mathrm{Q} 7_{\mathrm{c}}$, geologic error contributes significantly to the age spread of the sampled clasts. This may be explained by a variety of factors; the degree of scatter in a set of boulder/cobble ages from a younger surface-i.e. $\mathrm{Q} 1_{\ln }, \mathrm{Q} 2 \mathrm{a}_{\mathrm{c}}, \mathrm{Q} 2 \mathrm{~b}_{\mathrm{c}}, \mathrm{Q} 3 \mathrm{a}_{\mathrm{c}}$ - may be more easily influenced by clasts which contain significant inheritance and/or have experienced shielding.

In general, clasts which contain significant inheritance weigh more heavily in a dataset of a younger surface because the difference between individual clast ages and the true surfaces ages is minor; the clast ages obtained on younger surfaces may therefore overestimate the true age of the surface. Conversely, the influence of clast ages affected by inheritance is less significant on older surfaces, where the significantly older age of the surface essentially negates the contribution of inherited ${ }^{10} \mathrm{Be}$. In addition, older surfaces may also have a wider scatter of clast ages due to the work of erosion or exhumation of buried boulders and cobbles-particularly the well-developed desert pavements on these surfaces are evidenced to be deflational surfaces as discussed above. Individual clast ages on older surfaces may therefore underestimate true surface age. Each of these possibilities has implications for the use of $2 \sigma$ mean age as the surface age.

As discussed above, for the older $\mathrm{Q}_{\mathrm{c}}$ and $\mathrm{Q} 7_{\mathrm{c}}$ surfaces, significant deflation may have resulted in exhumation of clasts which have experienced copious shielding. This would cause the dataset to include clast ages much younger than a representative surface age. This seems to be a reasonable assumption to explain the anomalous ages in the Q7c dataset, as illustrated in Fig. 9. However, the spread of ages from the $\mathrm{Q}_{\mathrm{c}}$ is less easy to explain; the $2 \sigma$ outlier (ARQ6-3) is a much older sample than the other ages. This may be due to prior inheritance, although the amount of inherited ${ }^{10} \mathrm{Be}$ required to explain the age of this sample is significantly above that of the modern surface as suggested by depth profile analysis (discussed below) (Table 3). As such, the $\mathrm{Q}_{\mathrm{c}}$ dataset may illustrate the influence primarily of exhumation/shielding in the clast ages and secondary effect of inherited ${ }^{10} \mathrm{Be}$.

The $2 \sigma$ mean surface age method both provides ages for all but two $\left(\mathrm{Q}_{2} \mathrm{a}_{\mathrm{c}}\right.$ and $\left.\mathrm{Q}_{2} \mathrm{~b}_{\mathrm{c}}\right)$ alluvial fan/fluvial terrace surfaces and also best fits the geomorphic sequence of the surfaces, with only two exceptions ( $\mathrm{Q} 1_{\text {In }}$ and $\mathrm{Q} 1_{\mathrm{C}}$ ). We consider failed MSWD and PDF age results as cautions that even $2 \sigma$ mean ages for these surfaces may potentially be inaccurate.

Another possible interpretation of the ${ }^{10} \mathrm{Be}$ clast age results is to consider the age of the youngest cobble/boulder as most representative of the age of the surface. However, if erosion rate on a sampled clast is greater than our assumed rate $\left(0 \mathrm{~m} \mathrm{Ma}^{-1}\right)$ it may underestimate the true age of the clast. As discussed above, the potential error due to erosion rate varies significantly with our different reference erosion rates, and such error may be more significant than that of inheritance. Observation of cobble/boulder size on the Loma Negra alluvial fan surface indicates significant difference in clast size from the modern, $\mathrm{Q} 1_{\ln }$ surface to the oldest, $\mathrm{Q} 5$ In surface. Within the active channel large ( $>50 \mathrm{~cm}$ long-axis) boulders are present, whereas there are no boulders on the two oldest surfaces and nothing is $>14 \mathrm{~cm}$. 
A)

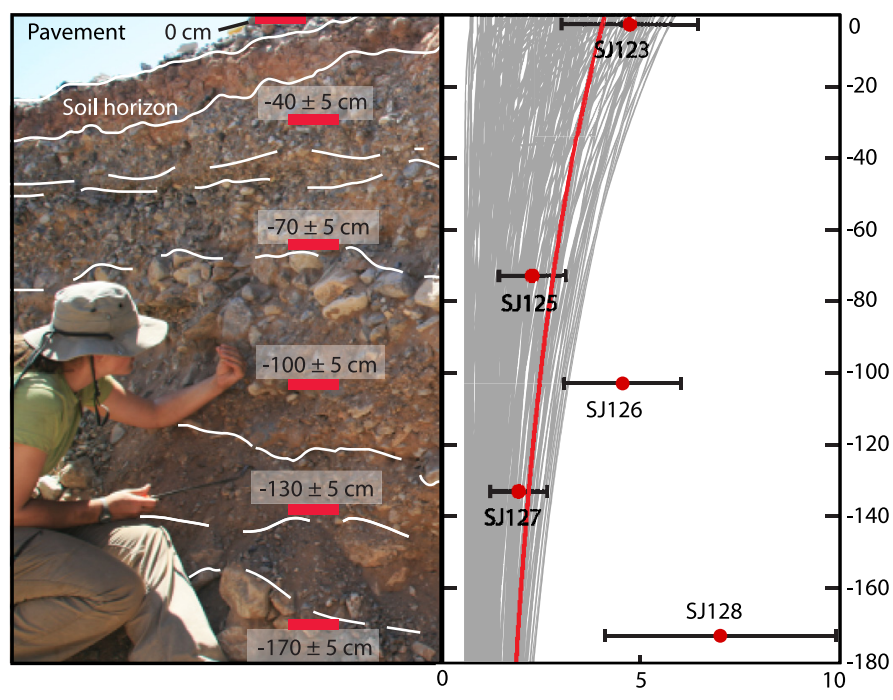

B)
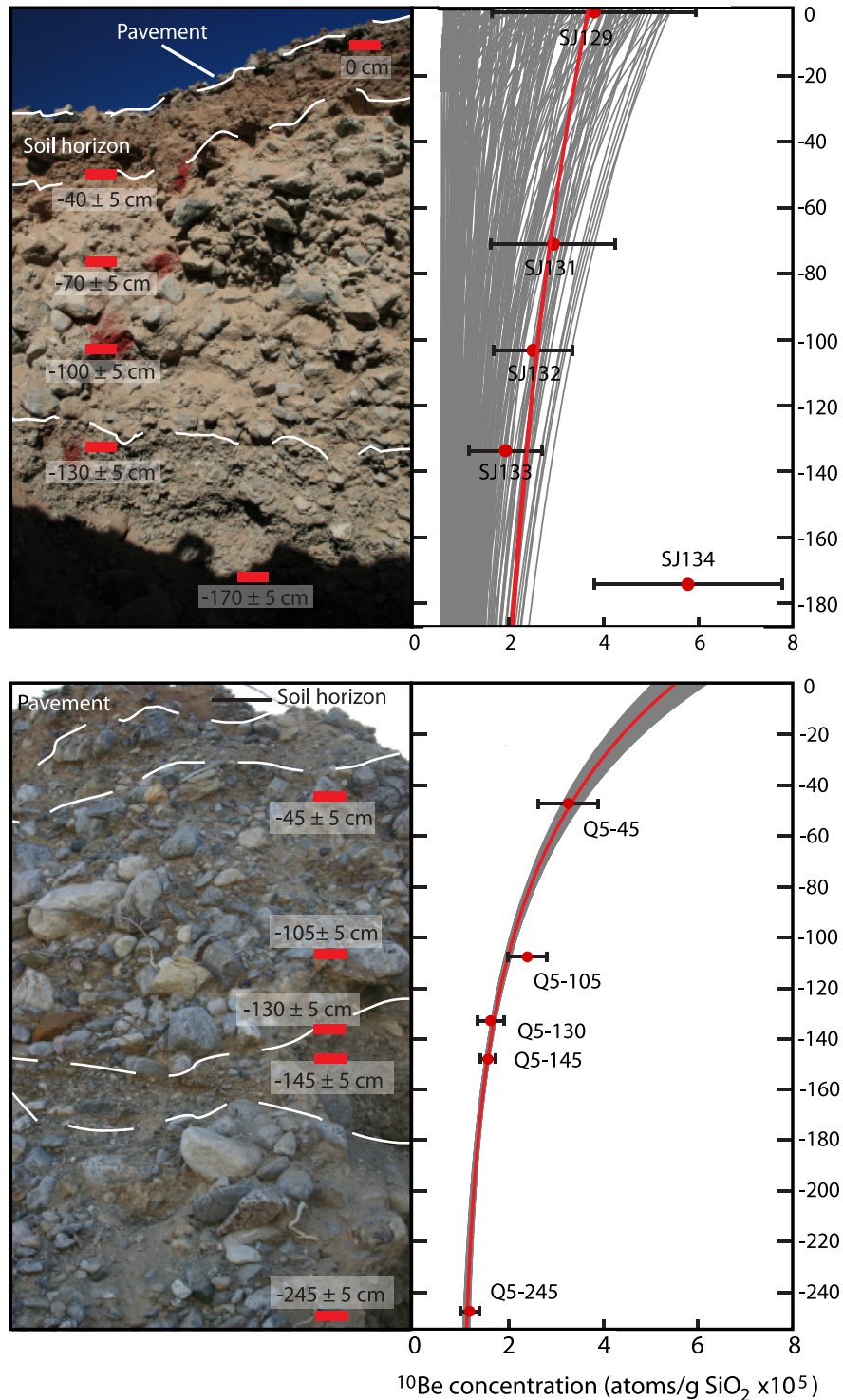
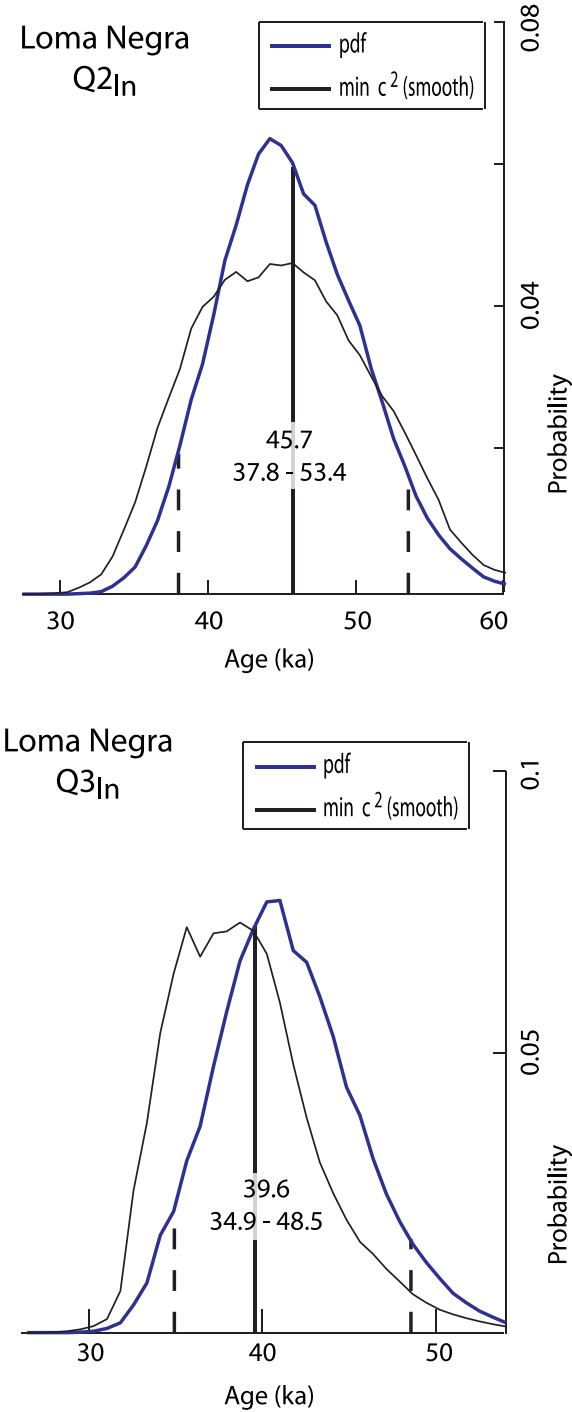

Carpintería

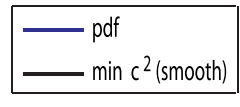

Q5c

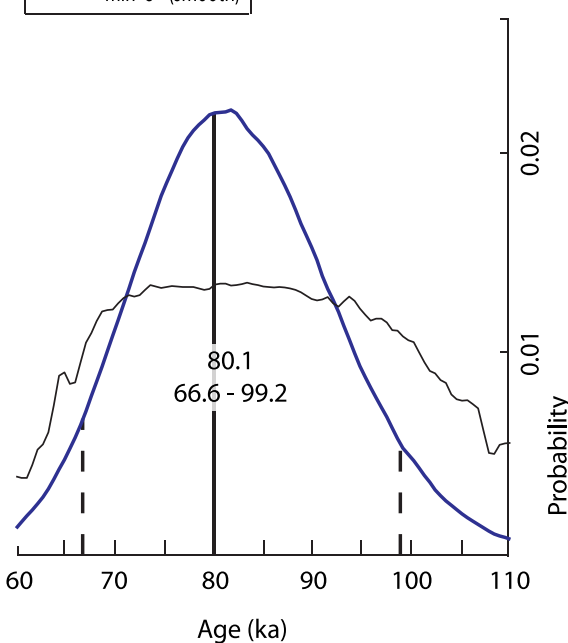

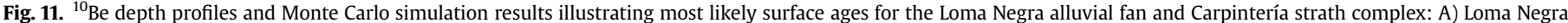
Q2 $\ln$ surface; B) Loma Negra Q3 $3_{\text {In }}$ surface; and C) Carpintería Q5 $5_{c}$ surface. 
This consistent and dramatic change in boulder size with increasing age may be primarily due to the erosional reduction in clast size of the lithologies that comprise the Loma Negra alluvial fan deposits-limestone with comparatively sparse chert veins. Boulders in the active channel are dominantly limestone with chert veins, whereas boulders and cobbles on the alluvial fan surfaces are dominantly chert with no or little limestone. This pattern seems to indicate weathering of chert out of limestone boulders over time; this also seems to be supported by boulder and cobble shape, which generally ranges from angular/sub-angular $\left(\mathrm{Q} 2{ }_{\ln }, \mathrm{Q}_{\ln }\right.$, and $\mathrm{Q} 4_{\ln }$ surfaces) with few truly rounded boulders (e.g. SJ105, SJ122, SJ136).

Due to the less resilient nature of limestone, the erosion rate of boulders on the Loma Negra alluvial fan surfaces is likely to change significantly with time; higher erosion rates can be expected on younger boulders where limestone dominates the composition of boulders and cobbles, while lower erosion rates are likely on surface of boulders/cobbles that are dominantly chert.

The concept of changing erosion rate on surface clasts seems to be supported by the above-mentioned distribution of maximum boulder sizes on the Loma Negra alluvial fan; we sampled the largest boulders present on Loma Negra alluvial fan surfaces (measurements in Table 1). From the $\mathrm{Q} 1_{\text {ln }}$ to $\mathrm{Q}_{\text {ln }}$ surfaces the maximum boulder size decreases from an average $36 \mathrm{~cm}$ to $\sim 14 \mathrm{~cm}$ in height, which ${ }^{10} \mathrm{Be}$ exposure age results suggest may reflect $\sim 40 \mathrm{ka}$ in time, whereas on the $\mathrm{Q} 4_{\ln }$ and $\mathrm{Q} 5_{\ln }$ surfaces, despite an age difference of $\sim 80 \mathrm{ka}$, the average cobble height is identical.

This non-linear rate of boulder size reduction seems to suggest a variable erosion rate based on boulder lithological composition. Marquesado strath terrace complex cobbles are the same lithology. However, the rocks that comprise the Carpintería strath terrace complex are primarily vein quartz and granite and may have experienced a different erosional history. Comparison with the OSL ages for the Carpintería $\mathrm{Q} 2 \mathrm{c}$ surface may have been helpful for this, but ${ }^{10} \mathrm{Be}$ exposure age results for that surface are less definitive of surface ages.

For our study, it is reasonable that the youngest age from both alluvial fan and strath terrace surfaces is not the most representative age. Instead, we suggest that, due to the remaining possibility of inheritance, surface ages should be considered by statistical means as $2 \sigma$ mean ages for the reasons discussed above. Although we note that this may underplay the significance of erosional and/ or exhumational processes in older surfaces, we stress that the underlying uncertainties (analytical and geological) make interpretation of these dates inherently somewhat difficult, and therefore define the ages of all surfaces as $2 \sigma$ mean age for all surfaces in order to remain consistent. Raw cobble and boulder ages are illustrated on all figures in order to allow the reader better comparison of sample and surface ages.

\section{3. ${ }^{10}$ Be depth profile results}

${ }^{10}$ Be depth profile data are summarized in Table 2, and Fig. 11 shows Hidy et al.'s (2010) Monte Carlo simulator results for depth profile plots along with likely values for inheritance, erosion rate, and surface age using 100,000 iterations. Depth profiles from both the $\mathrm{Q}_{2}$ an and $\mathrm{Q}_{3}$ In surfaces of the Loma Negra alluvial fan show significant scatter in ${ }^{10} \mathrm{Be}$ concentration at depth, which may indicate non-uniform inheritance from reactivation of previouslydeposited clasts with different depositional histories into new deposits.

Although the ${ }^{10} \mathrm{Be}$ concentrations of the Loma Negra Q2 In surface profile show significant scatter (Fig. 11A), the $\mathrm{Q}_{3}$ n surface profile indicates a reasonable decrease in ${ }^{10} \mathrm{Be}$ at depth save for the lowest sample at $170 \mathrm{~cm}$ (SJ134; Fig. 11B). This pattern of ${ }^{10} \mathrm{Be}$ concentration could form due to changes in the depositional environment from relative quiescence (allowing SJ134 to accumulate significant ${ }^{10} \mathrm{Be}$ concentration) to more regular depositional events. Q2 2 however reflects a more complex depositional/postdepositional history. Monte Carlo analysis suggests similar inheritance, erosion rate, and age values for both the $\mathrm{Q}_{2}$ In and $\mathrm{Q}_{3}$ In surfaces (Table 2). Bayesian most probable values of inheritance for Q2 $\mathrm{In}$ and $\mathrm{Q}_{3}$ In are $11.93 \times 10^{4}$ atoms and $11.61 \times 10^{4}$ atoms, respectively, and erosion rate values are 6.4 and $5.0 \mathrm{~m} \mathrm{Ma}^{-1}$, respectively. The modeled age of $\mathrm{Q}_{2} \ln$ is $41.8 \pm 7.4 \mathrm{ka}$ and $\mathrm{Q} 3_{\ln }$ is $38.8 \pm 6.0 \mathrm{ka}$, which suggests they are of approximately the same age (Fig. $11 \mathrm{~A}$ and $\mathrm{B}$, right columns). These values are within error of the $2 \sigma$ mean age for $\mathrm{Q} 2 \mathrm{ln}$, but are younger than the $2 \sigma$ mean age for the $\mathrm{Q} 3 \ln$ surface.

The depth profile for the Carpintería complex (on surface $Q 5_{c}$ ) is less complex and suggests a more uniform depositional environment. Analysis gives lower Bayesian most probable values for inheritance and erosion rate $\left(9.59 \times 10^{4}\right.$ atoms and $\left.3.8 \mathrm{~m} \mathrm{Ma}^{-1}\right)$ and a modeled surface age of $77.9 \pm 21.1 \mathrm{ka}$. This age is within error of the $2 \sigma$ mean.

The values for inheritance and erosion rate gleaned from this analysis may be used to further refine the ${ }^{10} \mathrm{Be}$-determined ages of surface cobbles and boulders. Although there is some agreement between depth profile results from the $\mathrm{Q} 2{ }_{\mathrm{ln}}, \mathrm{Q} 3_{\mathrm{ln}}$, and $\mathrm{Q} 5_{\mathrm{c}}$ surfaces, applying corrections for both inheritance and erosion rate presents difficulty as the accuracy of these values could be questioned; inheritance and erosion rate values were measured from pebbles in depth profiles, which may have experienced a different depositional history than the boulders and cobbles we sampled for surface age analysis. In addition, correction for inheritance brings ages on geomorphically older surfaces $\left(\mathrm{Q}_{\mathrm{m}}\right)$ to zero, which provides unlikely ages for these surfaces. Comparison with the Q2 $\mathrm{a}_{\mathrm{r}}$ and $\mathrm{Q} 2 \mathrm{~b}_{\mathrm{r}}$ ${ }^{10} \mathrm{Be}$ TCN and OSL ages shows that uncorrected ${ }^{10} \mathrm{Be}$ TCN clast ages agree more closely with OSL ages than inheritance-corrected ${ }^{10} \mathrm{Be}$ TCN clast ages.

As such we present ${ }^{10} \mathrm{Be}$ cobble and boulder ages revised for inheritance in Table 3 for the benefit of the reader, but do not utilize these revised age values in our statistical analyses, discussion of final results, and graphical representations of the data; we use the $2 \sigma$ mean age to define surface age. Due to the fact that error due to inheritance and erosion rate should be approximately equal across this small geographic region, the results of this study should be internally consistent and the ages relative to oneanother should be valid without inheritance correction. Additionally, as only one other study addressed in this paper reports ${ }^{10} \mathrm{Be}$ TCN results corrected for inheritance (Schmidt et al., 2011), we likewise do not do so in order to allow our best comparison with all results.

\subsection{OSL results}

The two OSL ages for the $\mathrm{Q} 2$ c surface for the Carpintería complex are $4.6 \pm 0.9 \mathrm{ka}$ (San Juan 1) and $4.9 \pm 0.5 \mathrm{ka}$ (San Juan 2) (Tables 3 and 4) (Fig. 9). Their internal consistency supports the likelihood that they closely approximate the actual age of the $\mathrm{Q} 2$ c deposits, however, they differ from many of the ${ }^{10} \mathrm{Be}$ TCN exposure ages of individual clasts on the $\mathrm{Q}_{\mathrm{c}}$ surfaces, which display large scatter. Our only option for comparison between ${ }^{10} \mathrm{Be}$ TCN ages and OSL ages for the $\mathrm{Q}_{\mathrm{c}}$ surfaces is the surface age obtained from PDF plots. These ages agree closely, with the ${ }^{10} \mathrm{Be}$ TCN surface age at $6.0 \pm 1.0 \mathrm{ka}$, which falls within error of both OSL ages. Although this comparison must be somewhat tentative given the large scatter of ${ }^{10} \mathrm{Be}$ TCN ages for this surface, an age of $\sim 5 \mathrm{ka}$ for this surface would not disagree with the geomorphic sequence of Carpintería terraces and seems like a reasonable estimate of the $\mathrm{Q}_{\mathrm{c}}$ surface age. 
Table 4

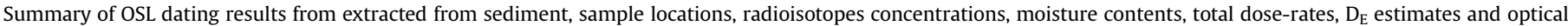
ages.

\begin{tabular}{|c|c|c|c|c|c|c|c|c|c|c|c|c|c|}
\hline Laboratory number & $\begin{array}{l}\text { Location } \\
\left({ }^{\circ} \mathrm{N} /{ }^{\circ} \mathrm{W}\right)\end{array}$ & $\begin{array}{l}\text { Altitude } \\
\text { ( } \mathrm{m} \text { asl) }\end{array}$ & $\begin{array}{l}\text { Depth } \\
(\mathrm{cm})\end{array}$ & $\begin{array}{l}\text { Particle } \\
\text { Size } \\
(\mu \mathrm{m})\end{array}$ & $\begin{array}{l}\mathrm{U}^{\mathrm{a}} \\
(\mathrm{ppm})\end{array}$ & $\begin{array}{l}\mathrm{Th}^{\mathrm{a}} \\
(\mathrm{ppm})\end{array}$ & $\begin{array}{l}\mathrm{K}^{\mathrm{a}} \\
(\%)\end{array}$ & $\begin{array}{l}\mathrm{Rb}^{\mathrm{a}} \\
(\mathrm{ppm})\end{array}$ & $\begin{array}{l}\text { Cosmic }^{\mathrm{b}, \mathrm{c}} \\
(\mathrm{Gy} / \mathrm{ka})\end{array}$ & $\begin{array}{l}\text { Dose-rate }{ }^{b, d} \\
(G y / k a)\end{array}$ & $\mathrm{n}^{\mathrm{e}}$ & $\begin{array}{l}\text { Mean equivalent dose }{ }^{f} \\
\text { (Gy) }\end{array}$ & $\begin{array}{l}\text { OSL Age } \\
\text { (ka) }\end{array}$ \\
\hline San Juan 1 & $31.788 / 68.576$ & 641 & 65 & $125-180$ & 2.2 & 7.65 & 1.79 & 79.1 & $0.22 \pm 0.02$ & $2.73 \pm 0.16$ & $15(46)$ & $12.61 \pm 0.16$ & $4.6 \pm 0.9$ \\
\hline San Juan 2 & $31.788 / 68.576$ & 641 & 73 & $90-125$ & 2.13 & 7.35 & 1.84 & 77.7 & $0.21 \pm 0.02$ & $2.73 \pm 0.17$ & $11(64)$ & $13.30 \pm 0.17$ & $4.9 \pm 0.5$ \\
\hline
\end{tabular}

a Elemental concentrations from NAA of whole sediment measured at USGS Nuclear Reactor Facility in Denver. Uncertainty taken as $\pm 10 \%$.

b Estimated fractional present-day water content for whole sediment is taken as $10 \pm 5 \%$.

c Estimated contribution to dose-rate from cosmic rays calculated according to Prescott and Hutton (1994). Uncertainty taken as $\pm 10 \%$.

d Total dose-rate from beta, gamma and cosmic components. Beta attenuation factors for U, Th and K compositions incorporating grain size factors from Mejdahl (1979).

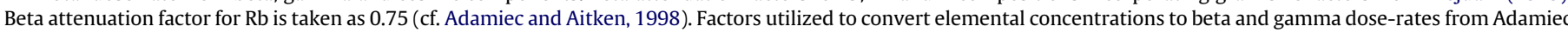
and Aitken (1998) and beta and gamma components attenuated for moisture content.

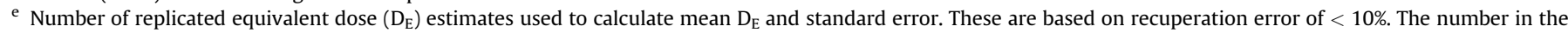
parenthesis is the total measurements made including failed runs with unusable data.

${ }^{\mathrm{f}}$ Mean equivalent dose $\left(\mathrm{D}_{\mathrm{E}}\right)$ determined from replicated single-aliquot regenerative-dose (SAR; Murray and Wintle, 2000) runs. The uncertainty also includes an error from beta source estimated of $\pm 5 \%$.

$\mathrm{g}$ Uncertainty incorporate all random and systematic errors, including dose rates errors and uncertainty for the $\mathrm{D}_{\mathrm{E}}$.

\section{Discussion}

\section{1. ${ }^{10}$ Be surface ages}

${ }^{10} \mathrm{Be}$ surface ages reveal a well-defined and consistent record of alluvial fan surface deposition and abandonment. All Loma Negra alluvial fan surface ages stratigraphically fit the geomorphic sequence of the alluvial fan surfaces, and the gap between surface ages seems to decrease over time. This is also seen in the Carpintería strath terrace complex surface age data, although there is overlap between the $\mathrm{Q6}_{\mathrm{c}}$ and $\mathrm{Q} 5_{\mathrm{c}}$ surface ages, as well as the $\mathrm{Q} 3 \mathrm{a}_{\mathrm{c}}$, $\mathrm{Q}^{3} \mathrm{~b}_{\mathrm{c}}$, and $\mathrm{Q} 1_{\mathrm{c}}$ surface ages. The Marquesado strath terrace complex surface $\left(\mathrm{Q}_{\mathrm{m}}\right)$ is the youngest of all dated surfaces.

The general trend of this robust data set suggests a potential correlation between along-strike surfaces in close proximity to one-another; surface ages from the three field sites overlap at

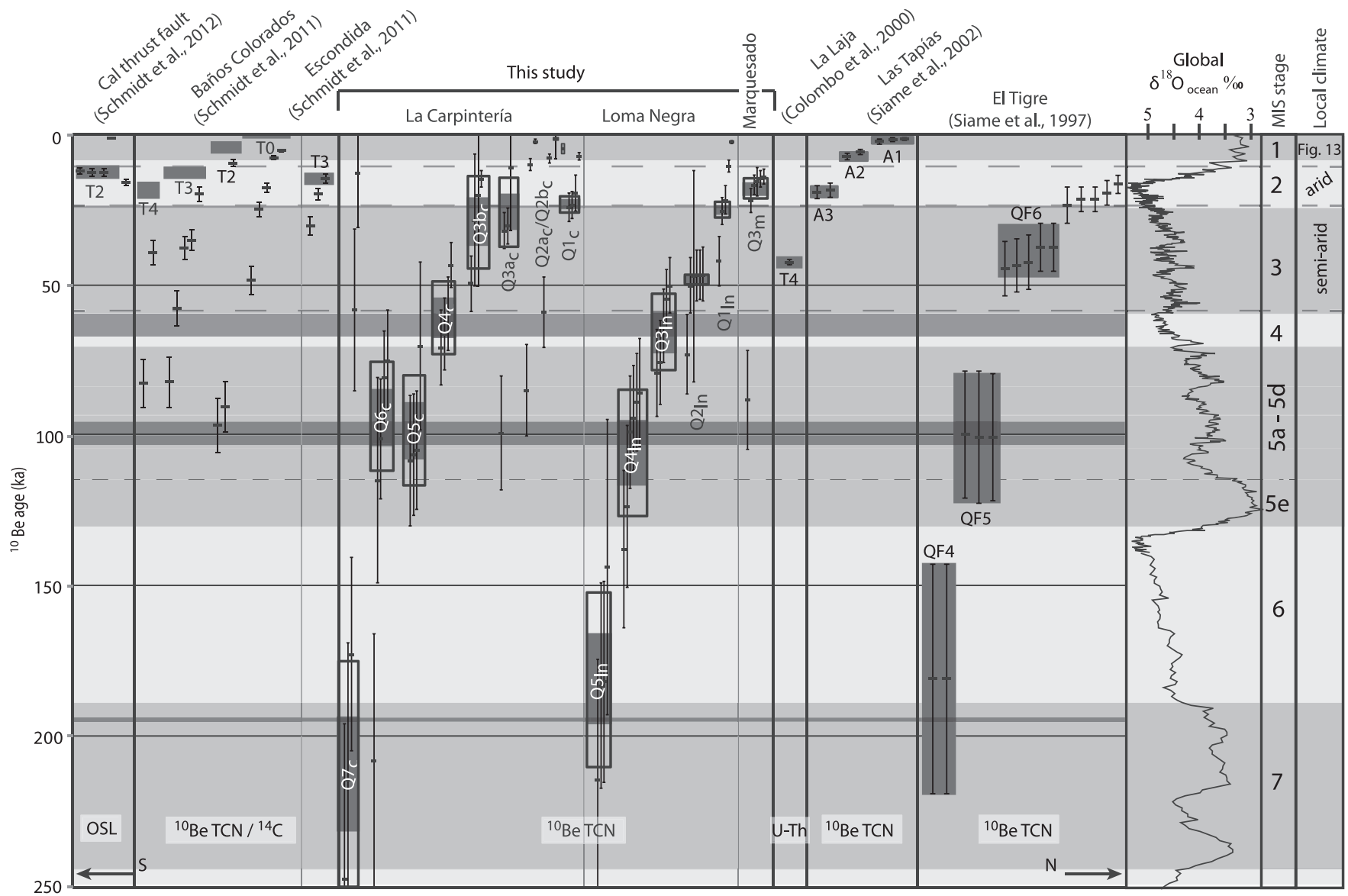

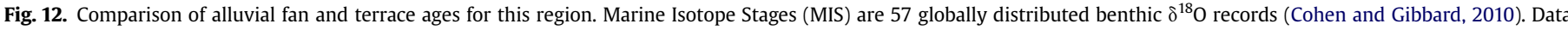

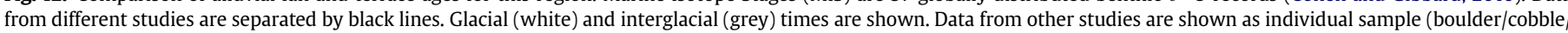

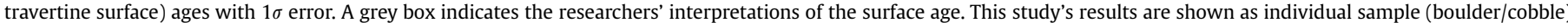

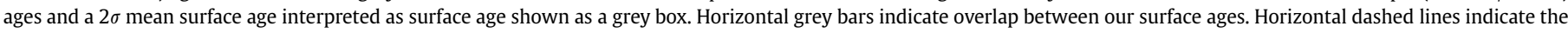
timing of local climatic shifts. 
180-200 ka, 85-110 ka, 45-70 ka, and 22-25 ka, ages which include all but one alluvial fan surface $\left(\mathrm{Q}_{\ln }\right)$ (Fig. 12).

Overlapping ages of the $\mathrm{Q}_{\mathrm{c}}$ and $\mathrm{Q} 5_{\mathrm{c}}$ surfaces as well as the $\mathrm{Q} 3 \mathrm{~b}_{\mathrm{c}}$ and $\mathrm{Q}^{3} \mathrm{a}_{\mathrm{c}}$ surfaces may indicate that deposition and/or abandonment of these terraces was approximately coeval. This is a reasonable assumption for the $\mathrm{Q}^{3} \mathrm{a}_{\mathrm{c}}$ and $\mathrm{Q}_{3} \mathrm{~b}_{\mathrm{c}}$ terraces in particular, as they are of nearly the same height and share similar characteristics with regard to development of desert pavement and varnish (Fig. 6).

The validity of this assumption is less clear with the $\mathrm{Q}_{\mathrm{c}}$ and $\mathrm{Q} 5_{\mathrm{c}}$ surfaces, which display varied development of desert varnish (Fig. 6) and are significantly different in height $(\sim 10 \mathrm{~m}) .{ }^{10} \mathrm{Be} \mathrm{TCN}$ depth profile results suggest a younger age for the $\mathrm{Q}_{\mathrm{c}}$ surface $(77.9 \pm 21.1 \mathrm{ka})$ than the $\mathrm{Q}_{\mathrm{c}} 2 \sigma$ mean surface age $(93.4 \pm 18.4 \mathrm{ka})$, although this still suggests ages that are well within error of oneanother. The Q6 r $_{\text {r }}$ surface also failed MSWD analysis, which suggests that the spread in surface clast ages are likely due to geologic error. Based on geomorphic evidence discussed above it is most likely that there is a significant difference in the true $\mathrm{Q}_{\mathrm{c}}$ and $\mathrm{Q6}_{\mathrm{c}}$ surface ages.

\subsection{Depth profiles}

Depth profiles reveal a more disordered stratigraphy on the Loma Negra alluvial fan than on the Carpintería surfaces. The Carpintería $\left(\mathrm{Q}_{\mathrm{c}}\right)$ depth profile reveals generally massive and unsorted units of diamicton with subrounded cobbles and sharp erosional contacts, whereas the Loma Negra depth profiles ( $2_{2}$ and $\left.{ }^{Q} 3_{\ln }\right)$ show variable grain sizes but are bedded and exhibit imbricated cobbles and pebbles and similarly sharp contacts. This may be due to a difference in the depositional and erosional processes at work on the two alluvial fans; the Carpintería strath terrace surfaces are at a greater distance from the range front $(\sim 3 \mathrm{~km}$ compared to
$<1 \mathrm{~km}$ on the Loma Negra alluvial fan) and receive sediment primarily through fluvial action. Due to the closer proximity to the range front, the Loma Negra alluvial fan surfaces may undergo higher-energy depositional and erosional processes than the Carpintería strath terrace complex.

\subsection{OSL}

The OSL ages from the $\mathrm{Q}_{\mathrm{c}}$ surface agree very well with oneanother and are statistically the same age at $\sim 4 \mathrm{ka}$. Although they are difficult to compare directly with the ${ }^{10} \mathrm{Be}$ ages for the Q2 $\mathrm{a}_{\mathrm{c}}$ and $\mathrm{Q}_{2} \mathrm{~b}_{\mathrm{c}}$ surfaces due to the scattered nature of those clast ages, they may help to clarify the ${ }^{10} \mathrm{Be}$ ages (Figs. 9 and 12). Although two samples from the Q2 $\mathrm{a}_{\mathrm{c}}$ and $\mathrm{Q}_{2} \mathrm{~b}_{\mathrm{c}}$ surfaces (ArQ2a-5 and ArQ2b-4) overlap at $\sim 3 \mathrm{ka}$ and fall within error of the OSL ages, the significant error of sample ARQ2a-5 (2.0 \pm 3.4$)$ makes this a very uncertain age estimate. The PDF of the $\mathrm{Q} 2 \mathrm{a}_{\mathrm{C}}$ surface provides an age of $\sim 6 \mathrm{ka}$, but is also reliant on sample ARQ2a-5. Given that the $2 \sigma$ mean age of both $\mathrm{Q} 2{ }_{\mathrm{c}}$ surfaces $(2.5 \pm 2.2)$ and PDF age (6.0 \pm 1.0$)$ are proxies for surface abandonment age whereas OSL ages (mean $=4.8 \pm 0.3$ ) roughly define sediment burial age, OSL ages should be older than or the same age as ${ }^{10} \mathrm{Be}$ TCN ages. This inversion of ages suggests that ${ }^{10} \mathrm{Be}$ surface ages are not valid for this surface. The fact that ${ }^{10} \mathrm{Be}$ TCN clast ages are dominantly much older than OSL ages suggests that inheritance is a major contributor to some of the measured clast ages.

\subsection{Comparison with other studies and further implications}

Other quantitative age studies of alluvial fans in this region include alluvial fan surfaces cut by the El Tigre fault $\sim 50 \mathrm{~km}$ to the northwest of our field area (Siame et al., 1997) and the Las Tapías alluvial fan on the Villicum-Zonda thrust $\sim 15 \mathrm{~km}$ northeast along

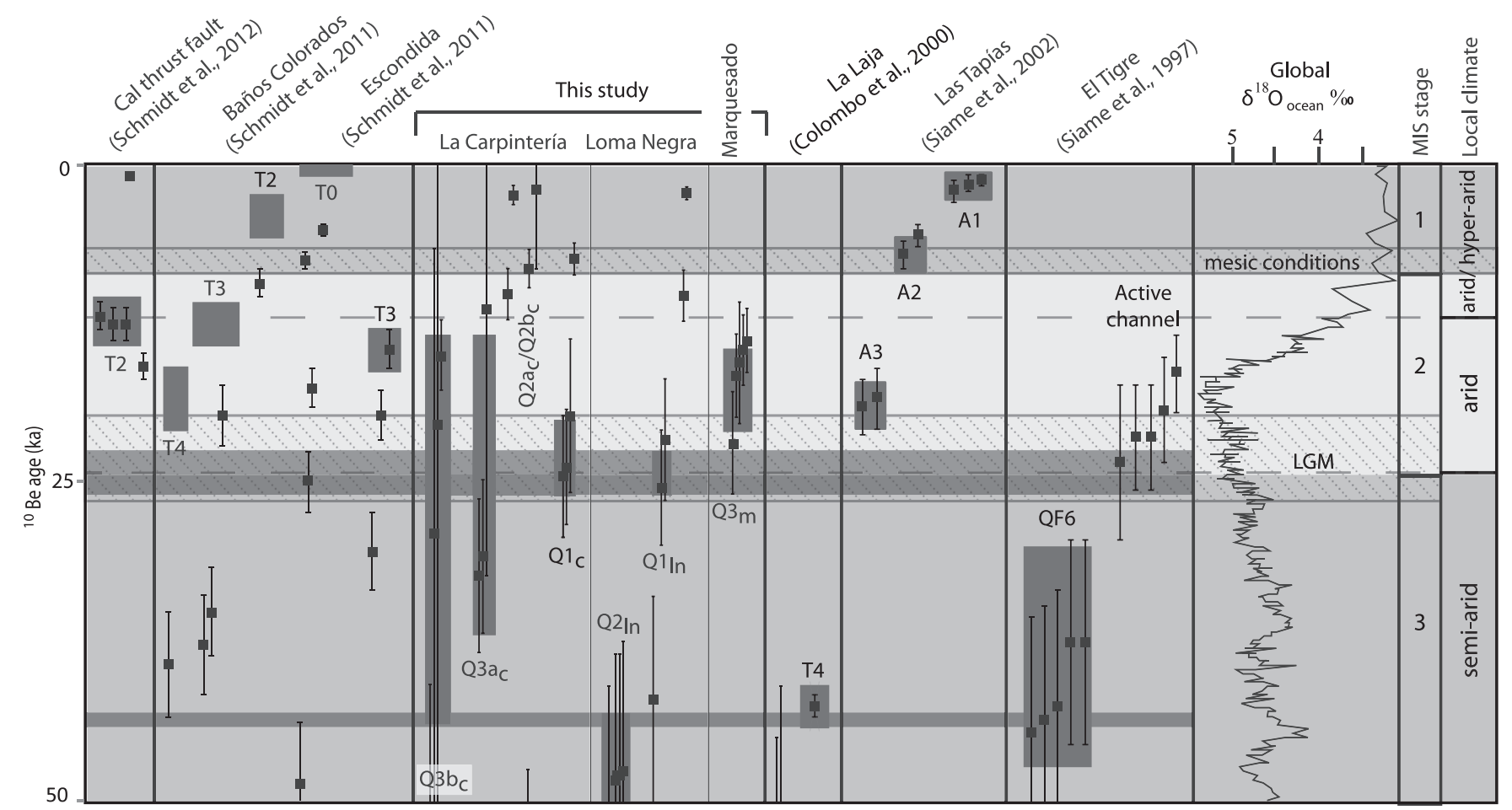

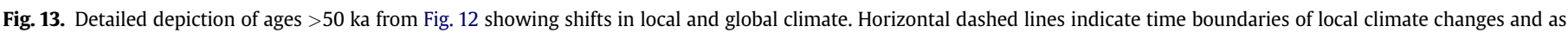

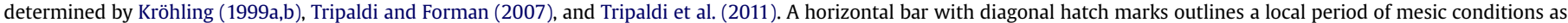

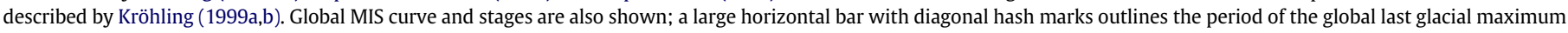
(LGM). The horizontal grey bars indicate periods of surface age overlap for this and previous studies. 
the same range front as our field areas (Siame et al., 2002). Although these studies focused primarily on the use of alluvial fan surface dating for the purpose of calculating slip rates of the El Tigre and Villicum-Zonda faults, the ages provided for the alluvial fan surfaces suggest a consistent history of alluvial fan surface deposition and abandonment.

Results from Siame et al. (1997) and Siame et al. (2002) are plotted in Fig. 12 as individual samples (bars with error) and the investigators' interpretation of the age data (grey boxes). Siame et al. (1997) suggest that minimum ages on each surface are most accurate, resulting in surface ages of $670.0 \pm 140.0 \mathrm{ka}$ and $380.0 \pm 78.0 \mathrm{ka}($ not shown), $180.0 \pm 38.0 \mathrm{ka}, 100.0 \pm 21.0 \mathrm{ka}$, and $41.0 \pm 8.5 \mathrm{ka}$. Siame et al. (2002) use either the maximum (A1 and A2 surfaces) or the average (A3 surface) surface ages to determine alluvial fan surface formation at $18.7 \pm 2.3 \mathrm{ka}, 6.8 \pm 1.0 \mathrm{ka}$, and $1.5 \pm 0.8 \mathrm{ka}$.

Although Siame et al. (1997) do not clarify what ${ }^{10}$ Be production rate was used for their age calculations, Siame et al. (2002) indicate that they used a reference production rate of 5.1 atoms $\mathrm{g}^{-1} \mathrm{yr}^{-1}$ and calculated their ages using the Lal (1991)/Stone (2000) timeindependent scaling scheme, whereas we use the Lal (1991)/ Stone (2000) time-dependent scaling scheme and a production rate calculated under modern standards. Using the revised production rate of 4.39 atoms $\mathrm{g}^{-1} \mathrm{yr}^{-1}$ results in a $\sim 14 \%$ decrease in production rate and thus decreases sample ages by $\sim 14 \%$. Additionally, the use of the Lal (1991)/Stone (2002) time-independent scaling scheme rather than the Lal (1991)/Stone (2002) time-dependent scaling scheme may affect the interpretation of surface ages. Using our own raw data, we find that calculating ages using the Lal (1991)/Stone (2002) time-dependent scaling scheme lowers time-independent ages of samples $\geq 50$ ka by $\sim 10 \%$ with younger samples being less affected by the change-an age of $\sim 25 \mathrm{ka}$ is lowered by $\sim 3.5 \%$. Calculating ages using the Lal (1991)/Stone (2002) time-dependent scaling scheme does not significantly affect any surface ages from Siame et al. (2002), although it does have a greater effect on Siame et al.'s (1997) older surface ages. We discuss potential issues due to these corrections in the context of our own results below.

Colombo et al. (2000) investigated an alluvial fan terrace at La Laja, $\sim 15 \mathrm{~km}$ northeast of our field sites. They sampled a travertine deposit overlying a Quaternary (T4) alluvial fan terrace. Using $\mathrm{U}-\mathrm{Th}$ dating, they determine an age of $42.0 \pm 1.0 \mathrm{ka}$ (Fig. 12).

Schmidt et al. (2011) investigated two alluvial fans $\sim 80 \mathrm{~km}$ to the south of our field areas using ${ }^{10} \mathrm{Be}$ TCN boulder exposure age and radiocarbon methods. At both alluvial fan sites they also attempt to determine the amount of inheritance present in their samples using ${ }^{10} \mathrm{Be}$ depth profile analysis and ${ }^{10} \mathrm{Be}$ TCN amalgamated pebble samples. They apply their inheritance results in their interpretation of their boulder ages; Fig. 12 displays Schmidt et al.'s (2011) individual boulder ages (bars with error) as well as their final interpretation of the alluvial fan surface ages (grey boxes). Using similar ${ }^{10} \mathrm{Be}$ age calculation methods to this study, they suggest alluvial fan surface formation at $12 \pm 2 \mathrm{ka}$ (T3) at the Escondida site and at $18 \pm 2 \mathrm{ka}$ (T4), $12 \pm 1 \mathrm{ka}$ (T3), and $4 \pm 1 \mathrm{ka}$ (T2) at the Baños Colorados site. $\mathrm{A}^{14} \mathrm{C}$ age of $12.6 \pm 0.2 \mathrm{ka}$ was used as calibration for the T3 ${ }^{10}$ Be age.

Schmidt et al.'s (2011) active channel ${ }^{10} \mathrm{Be}$ inheritance was determined to be $7.24 \pm 0.34 \times 10^{4}$ atoms $g^{-1}$. Schmidt et al. (2011) also determined inheritance from sand collected from depth profiles on younger surfaces which produce much lower values of inheritance $\left(1.19 \pm 0.31\right.$ to $2.19 \pm 0.87 \times 10^{4}$ atoms $\left.\mathrm{g}^{-1}\right)$ but suggest that this difference is likely due to differing source area and transport histories of different grain sizes. This active channel inheritance value is slightly less than the inherited ${ }^{10} \mathrm{Be}$ concentration we determined at both the Carpintería complex $\left(9.5 \pm 1.4 \times 10^{4}\right.$ atoms $\left.\mathrm{g}^{-1}\right)$ and the Loma Negra alluvial fan $\left(11.9 \pm 2.9 \times 10^{4}\right.$ atoms $g^{-1}$ and $11.6 \pm 1.4 \times 10^{4}$ atoms $\left.^{-1}\right)$.
We briefly investigated the relationship between inherited ${ }^{10} \mathrm{Be}$ and distance from source area to sampling site using our data and the data from Schmidt et al. (2011), but found no correlation.

Schmidt et al. (2012) also investigated alluvial fans using OSL and radiocarbon methods $\sim 130 \mathrm{~km}$ to the south of our field areas. Using minimum age models to interpret their data, they suggest that alluvial fan sediments there were deposited $\sim 12$ ka (Fig. 12). They further suggest that these deposits coincide with a phase of cool and humid climate linked to glacial melt (c.f. D’Antonio, 1983; Markgrav, 1989; García et al., 1999). However, the presence of gypsum in all soil profiles in this study precludes a humid environment.

We provide our best summary of all raw alluvial fan and fluvial/ strath terrace surface age data for this region, and individual researchers' interpretation of that data, in Fig. 12. The ages of our alluvial fan and strath terrace surfaces $\left(\mathrm{Q} 4_{c}-\mathrm{Q} 1_{c} ; \mathrm{Q} 4_{\ln }-\mathrm{Q} 1_{\ln }\right)$ generally differ markedly from those obtained on Schmidt et al. (2011), Schmidt et al. (2012) and Siame et al.'s (2002) chronologically identical surfaces (T4-T0 and A3-A1, respectively), both in the raw data and in the researchers' age interpretations. Our surface ages are much older than those of Schmidt et al. (2011, 2012), and Siame et al. (2002), which has significant implications for the interpretation of Quaternary fault slip rates.

However, as discussed above, recalculation of Siame et al. (1997) and Siame et al.'s (2002) sample ages using modern production rate (4.39 atoms $\mathrm{g}^{-1} \mathrm{yr}^{-1}$ ) and the Lal (1991)/Stone (2000) timedependent scaling scheme generally causes no change in agreement with our dataset. The only exception are the ages of Siame et al.'s (1997) QF5 surface; given that the new age of the QF5 samples fall within a range of $85-86 \mathrm{ka}$, in addition to the $\sim 14 \%$ decrease in age due to change in production rate, they also are affected by $\sim 10 \%$ decrease in age due to the use of different scaling schemes. Even with these corrections Siame et al.'s (1997) QF5 still falls within error of our Q4 $4_{\ln }$ and $\mathrm{Q} 5_{\mathrm{c}}$ surfaces, and agrees better with our $\mathrm{Q6}_{\mathrm{c}}$ surface as seen in Fig. 12. The large error associated with these ages paired with issues of sample age scatter due to processes such as boulder/cobble exhumation and erosion already make interpretation of surface ages tentative.

The results of Schmidt et al. (2011) in particular illustrate considerable scatter in sample ages, which suggests the ultimate interpretation of surface age must be carefully considered and may be unreliable. Similarly, a statistically significant population $(\geq 3)$ of overlapping sample ages is necessary to be confident in the final interpretation of surface age results. For many surfaces and studies (Colombo et al., 2000; Siame et al., 2002; Schmidt et al., 2011) this condition is not met.

Most datasets to which we compare our own do not/cannot pass MSWD analysis and do not yield a viable $2 \sigma$ mean age. Therefore, direct comparisons between datasets must be undertaken with care. This problem is primarily due to the general paucity of surface age dates in this area, both within individual studies and as a whole; surfaces must be sampled thoroughly enough to provide a statistically viable population of overlapping dates in order to reliably determine a surface age, different methods may return different results and those results must be interpreted differently, and all methods have inherent weaknesses which may complicate age interpretation. These varied issues make regional comparisons of data tentative, but consistent patterns may still yield useful insights.

Alluvial fan and strath terrace surface ages presented in this study, which are internally consistent, correlate strongly as illustrated in Fig. 12 (dark grey, horizontal bars). Significant overlap in ages between our field areas occurs at $\sim 200-180 \mathrm{ka}$, $\sim 110-85 \mathrm{ka}, \sim 70-45 \mathrm{ka}$, and $\sim 25-22 \mathrm{ka}$. Both the $\sim 200-$ $180 \mathrm{ka}$ and $\sim 110-85 \mathrm{ka}$ periods of alluvial fan and fluvial terrace 
surface abandonment also correlate to Siame et al.'s (1997) Q4 and QF5 surfaces. Larger error on Siame et al.'s (1997) QF4 surface combined with the error of our $\mathrm{Q} 5_{\ln }$ and $\mathrm{Q} 7_{\mathrm{c}}$ surfaces makes this correlation less strong, but there is a very strong correlation between our Q5, Q4 $4_{\text {In }}$, and Siame et al.'s (1997) QF5 surface at $\sim 100 \mathrm{ka}$. This general pattern of overlap is not broken by any reasonable interpretation of the Siame et al. (1997) data nor by applying our inheritance corrections to our data, which decreases our surface ages by $\sim 10 \mathrm{ka}$.

Interpreting the overlap of younger ages presents more difficulty due to the potential influence of inheritance and/or erosion on sample ages. A decrease of $\sim 10 \mathrm{ka}$ in our youngest surface ages due to potential inheritance, for example, may cause our dates to more closely align with any of Siame et al.'s (2002), Schmidt et al.'s (2011), or Schmidt et al.'s (2012) surface ages. However, due to the larger error in our $\mathrm{Q}^{3} \mathrm{a}_{\mathrm{c}}$ and $\mathrm{Q} 3 \mathrm{~b}_{\mathrm{r}}$ ages this comparison would already be tentative. Generally our data are too young to correlate with those of other studies, although our youngest dated surface (Q3 $3_{\mathrm{m}}$ ) correlates well with Siame et al.'s (2002) A3 surface. Exclusion of our dataset in this regional comparison for surface ages $<25$ ka still reveals overlap between Schmidt et al.'s $(2011,2012)$ T2 surface on the Cal thrust fault, T3 surface on the Baños Colorados site, and the T3 surface of the Escondida site. These ages are only slightly younger than our $\mathrm{Q} 3 \mathrm{~m}$ and Siame et al.'s (2002) A3 surfaces.

Climate records focused on Argentina are typically located a significant distance from our study area and/or reach back over a very short timescale ( $<8 \mathrm{ka}$ ) (Mancini et al., 2005; Tripaldi and Forman, 2007; Morales et al., 2009) and are thus too young to be applied to our dataset. However, we compare our alluvial fan age data to the global MIS as compiled by Cohen and Gibbard (2010; Figs. 12 and 13). Combined data from all studies seem to show significant alluvial fan deposition concentrated during MIS 2 and through MIS 1, potentially illustrating a period of enhanced fan surface formation after $\sim 25 \mathrm{ka}$. However, this trend also no doubt reflects the availability of alluvial fan data; in many studies (Colombo et al., 2000; Siame et al., 2002; Schmidt et al., 2012) terraces older than 25 ka were not dated. In addition, our dataset in combination with those of other researchers may illustrate a potential lack of preservation of surfaces; although the current arid environment suggests maximum preservation potential, past environmental changes may have influenced the preservation of alluvial fan and fluvial terrace surfaces over time.

Our data in combination with other studies within a climatically-similar region illustrates generally synchronous ages which also seem to be concentrated at times of established climate shift. The presence of correlating terrace surface ages over a broad geographic area favors the influence of climatic events as a forcing mechanism over a tectonic influence. This suggestion is further supported by the alignment of many surface ages $(\sim 100 \mathrm{ka}$, $\sim 50 \mathrm{ka}, \sim 25 \mathrm{ka}$ ) with known periods of climatic shift. However, it is difficult to determine the exact reason for terrace abandonment at these ages; well-established climate records for these regions are somewhat limited temporally and do not provide information beyond $\sim 60 \mathrm{ka}$. These records also generally illustrate only shifts between arid and semi-arid climate. The suggested relationship of terrace abandonment ages with climatic shifts nevertheless remains strong.

\section{Conclusion}

Our study provides the first expansive surface ages for alluvial fan and strath terrace surfaces in the Eastern Precordillera of Argentina: the Marquesado terrace complex, the Loma Negra alluvial fan, and the Carpintería terrace complex. One surface age $\left(\mathrm{Q} 3_{\mathrm{m}}\right)$ was obtained on the Marquesado complex ( $\left.\sim 17 \mathrm{ka}\right)$, whereas the dating of multiple surfaces on Loma Negra (Q1 $1_{\text {In }}$ through $\mathrm{Q}_{\mathrm{In}}$ ) and Carpintería $\left(\mathrm{Q}_{\mathrm{c}}\right.$ through $\mathrm{Q} 3_{\mathrm{c}}$ ) show a clear and consistent sequence of depositional events. On the Carpintería complex, the youngest $\mathrm{Q} 1_{\mathrm{c}}$ and $\mathrm{Q} 2_{\mathrm{c}}$ surfaces provide scattered data, potentially due to significant reworking of clasts in the active channel ( $1_{\mathrm{c}}$ ) and lowest terrace ( $\left.\mathrm{Q}_{\mathrm{c}}\right)$. OSL data indicate an age of $\sim 5 \mathrm{ka}$ for the Carpintería $\mathrm{Q} 2 \mathrm{c}$ surfaces.

Surface ages, excepting the Carpintería $\mathrm{Q} 2 \mathrm{c}$ and $\mathrm{Q} 1_{\mathrm{c}}$ surfaces, match relative ages as determined with geomorphic mapping. The age of the Marquesado $\mathrm{Q}_{\mathrm{m}}$ surface is younger than that of the Loma Negra and Carpintería $\mathrm{Q} 1_{\mathrm{ln}}$ and $\mathrm{Q} 1_{\mathrm{c}}$ surfaces; this suggests that the Marquesado terrace complex is a much younger and potentially more active landform, having either undergone significantly more periods of deposition surfaces over the past $\sim 17 \mathrm{ka}$ than either the Loma Negra fan or Carpintería terraces or experienced better preservation of those depositional surfaces.

Ages from the Marquesado complex - which is traversed by four distinct, high fault scarps-suggest more frequent tectonism along the Sierra de Marquesado, where the Marquesado terraces are located, than along the Sierra Chica de Zonda, along which the Loma Negra alluvial fan and Carpintería complex have formed. However, this is unlikely as the Marquesado complex is near the termination of the northern segment of the Sierra Villicum, where deformation steps southward and to the west of the Sierra Chica de Zonda. As only one surface was sampled from the Marquesado complex $\left(\mathrm{Q} 3_{\mathrm{m}}\right)$, more work is needed to confidently determine Marquesado surface ages and tectonic history.

In addition, ages obtained for the Marquesado complex $\mathrm{Q}_{\mathrm{m}}$ surface show that the formation of all fault scarps on that alluvial fan occurred after $20 \mathrm{ka}$, whereas on the Loma Negra alluvial fan the age of the $\mathrm{Q} 2$ In surface shows that the faulting may be as old as $50 \mathrm{ka}$. The relationship between alluvial fan surfaces and fault scarps is less clear on the Carpintería alluvial fan; although a sand bar within the active channel appears to have been cross-cut by a fault, the active channel $\left(\mathrm{Q} 1_{\mathrm{c}}\right)$ in which ages were obtained does not show the presence of a fault. Defining fault rupture ages on the Carpintería alluvial fan is made difficult by the inconsistent ${ }^{10} \mathrm{Be}$ exposure ages of the higher $\mathrm{Q}_{\mathrm{c}}$ surface. OSL data obtained from the Q2 c surface suggests an age of $\sim 5 \mathrm{ka}$.

Depth profiles measured on the Loma Negra Q2 1 and $\mathrm{Q}_{\text {In }}$ surfaces indicate that inheritance in samples may be significant $\left(\sim 12 \times 10^{4}\right.$ atoms $\left.\mathrm{g}^{-1}\right)$, particularly for samples with a young age, although the ages suggested for these surfaces match well with surface sample age data. Additionally, erosion rates on the Loma Negra Q2 In and Q3 3 surfaces as determined by depth profile are $\sim 5 \mathrm{~m} \mathrm{Ma}^{-1}$, which is a reasonable value for desert environments. Bedrock exposures from the Loma Negra source area reveal a similar erosion rate of $\sim 6 \mathrm{~m} \mathrm{Ma}^{-1}$. These values differ significantly from the erosion rate of both the catchment and hillslope, which also vary between samples (hillslope erosion rates are $\sim 11 \pm 1 \mathrm{~m} \mathrm{Ma}^{-1}$ and $\sim 24 \pm 5 \mathrm{~m} \mathrm{Ma}^{-1}$, catchment erosion rates are $\sim 49 \pm 7 \mathrm{~m} \mathrm{Ma}^{-1}$ and $\sim 140 \pm 20 \mathrm{~m} \mathrm{Ma}^{-1}$ - which are below the worldwide average erosion rates for tectonically active basins $\left(367 \pm 55 \mathrm{~m} \mathrm{Ma}^{-1}\right.$ ) but slightly above that of arid regions $\left(100 \pm 17.3 \mathrm{~m} \mathrm{Ma}^{-1}\right)$ (Portenga and Bierman, 2011).

The depth profile measured on the Carpintería $Q 5_{c}$ alluvial fan surface suggests slightly lower values for both inheritance and erosion rate of $\sim 10 \times 10^{4}$ atoms $\mathrm{g}^{-1}$ and $3.8 \mathrm{~m} \mathrm{Ma}^{-1}$, respectively. The age for the Carpintería $\mathrm{Q}_{\mathrm{c}}$ surface, as calculated by depth profile, is within error of the age determined by ${ }^{10} \mathrm{Be}$ surface exposure age samples. Corrections for inheritance would lower surface ages by $\sim 10 \mathrm{ka}$. This would not greatly affect interpretation of older surfaces, but cautions greater care is needed with interpretation of younger surface ages. It is important also to note that our inheritance values are measured from pebble samples, which 
may not accurately reflect the actual concentration of inherited

${ }^{10} \mathrm{Be}$ in our boulder and cobble samples.

Alluvial fans investigated in other studies generally reveal much younger ages for alluvial fan terraces (Siame et al., 2002; Schmidt et al., 2011, 2012) with the exception of ages along the El Tigre fault (Siame et al., 1997). The ages of alluvial fan surfaces to both the north and south of our field areas are generally restricted to $<25 \mathrm{ka}$, with significant fan deposition occurring in that short time (e.g. all fan terraces from Colombo et al., 2000; Siame et al., 2002; Schmidt et al., 2011, 2012).

Significant overlap of surface ages exists between not only our own alluvial fan and fluvial terrace dataset, but also between many surfaces investigated by other researchers, particularly those of Siame et al. (1997). Correlation of our Q6c, Q5 , and Q4 In surfaces with Siame et al.'s (1997) QF5 surface suggests significant alluvial fan and fluvial terrace surface abandonment over a broad spatial region at $\sim 100 \mathrm{ka}$. Overlap of our Q4 $\mathrm{c}$ and Q3 $3_{\text {In }}$ surface ages roughly correlate with an established shift to semi-arid conditions at $\sim 58 \mathrm{ka}$. Our younger $\mathrm{Q}^{3} \mathrm{~b}_{\mathrm{c}}, \mathrm{Q}^{3} \mathrm{a}_{\mathrm{c}}$ surfaces and the $\mathrm{Q} 1_{\mathrm{c}}$ and $\mathrm{Q} 1_{\mathrm{ln}}$ ages correlate strongly at a known climatic shift from semi-arid to arid at $\sim 24 \mathrm{ka}$, which corresponds to the beginning of MIS-2. This correlation must be considered somewhat tentative due to the potential influence of inheritance on our sample ages.

However, close correlation between our older ( $>50 \mathrm{ka}$ ) surface ages with those of the likewise inheritance-uncorrected ages of Siame et al. (1997) still suggest a broad regional pattern of alluvial fan and strath terrace surface formation and abandonment over long time scales. Although the inherent difficulties in interpreting sample sets with wide scatter make such a conclusion tentative, this broad regional agreement of surface ages better supports a theory of climatic forcing as the dominant cause for terrace formation in this region over the last $\sim 50 \mathrm{ka}$ rather than that of tectonic forcing. Higher resolution in alluvial fan ages, particularly measured by multiple methods, may help to illuminate the nature and timing of alluvial fan formation and better elucidate the potential link with climatic events illustrated by our data and that of other studies. Whether alluvial fan formation is related to higher-magnitude, low frequency events-possibly related to cyclical climate changes-or if alluvial fan deposition is caused by more consistent, higher frequency events is difficult to assess. Although our data seems to support the latter, more study is needed to elucidate the complex processes at work in the alluvial fan environment.

\section{Acknowledgments}

We acknowledge support from NSF grant EAR-0838344 and thank Jorge A. Ahumada and Escofet Agustin of the Loma Negra processing plant for access to field sites and use of their backhoe. Thanks also to Alan Hidy for his help with MATLAB code. We thank the three anonymous reviewers and editor Claude Hillaire-Marcel for their very constructive and useful comments that help us improve our manuscript.

\section{References}

Abraham, E.M., Garleff, K., Liebricht, H., Regairaz, A.C., Schaebitz, F., Squeo, F.A., Stingl, H., Villagrán, C., 2000. Geomorphology and paleoecology of the arid diagonal in southern South America. Zeitschrift für Angewandte Geologie SH1, 55-61.

Adamiec, G., Aitken, M., 1998. Dose-rate conversion factors: update. Ancient TL 16, $37-50$.

Alvarado, P., Beck, S., Zandt, G., 2007. Crustal structure of the south-central Andes Cordillera and backarc region from regional waveform modeling. Geophysical Journal International 170, 858-875.

Applegate, P.J., Urban, N.M., Laabs, B.J.C., Keller, K.., Alley, R.B., 2010. Modeling the statistical distributions of cosmogenic exposure dates from moraines. Geoscientific Model Development 3, 293-307.
Applegate, P.J., Urban, N.M., Keller, K., Lowell, T.V., Laabs, B.J.C., Kelly, M.A., Alley, R.B., 2012. Improved moraine age interpretations through explicit matching of geomorphic process models to cosmogenic nuclide measurements from single landforms. Quaternary Research 77, 293-304.

Balco, G., Briner, J., Finkel, R.C., Rayburn, J., Ridge, J.C., Schaefer, J.M., 2009. Regional beryllium-10 production rate calibration for late-glacial northeastern North America. Quaternary Science Reviews 4, 93-107.

Bastías, A., Henríquez, H., 1984. Afloramientos calcáreos en el borde oriental del valle del Bermejo, provincia de San Juan. Revista de la Asociación Geológica Argentina 39, 153-155.

Blair, T.C., McPherson, J.G., 1994. Alluvial fan processes and forms. In: Abrahams, A.D., Parsons, A.J. (Eds.), Geomorphology of Desert Environments. Chapman and Hall, London, pp. 354-402.

Boothroyd, J.C., Nummendal, D., 1977. Proglacial braided outwash - a model for humid alluvial-fan deposits. In: Miall, A.D. (Ed.), Fluvial Sedimentology. Canadian Society of Petroleum Geologists, Memoir, vol. 5, pp. 641-688.

Braucher, R., Del Castillo, P., Siame, L., Hidy, A.J., Bourlés, D.L., 2009. Determination of both exposure time and denudation rate from in situ-produced ${ }^{10} \mathrm{Be}$ depth profile: a mathematical proof of uniqueness. Model sensitivity and applications to natural cases. Quaternary Geochronology 4, 56-67.

Bruniard, E.D., 1982. La diagonal árida Argentina: un límite climático real. Revista Geográfica 95, 5-20.

Bull, W.B., 1977. The alluvial-fan environment. Progress in Physical Geography 1, 222-270.

Bull, W.B., 1991. Geomorphic Responses to Climatic Change. Oxford University Press, New York.

Capitanelli, R., 1972. Geomorphologia y clima de la provincial de Mendoza. Boletin de la Sociedad Argentina de Botanica, XIII Suplemento, 15-48.

Church, M., Ryder, J.M., 1972. Paraglacial sedimentation: a consideration of fluvial processes conditioned by glaciations. Geological Society of America Bulletin 83 (10), 3059-3072.

Church, M., Ryder, J.M., Eyles, N., Kocsis, S.P., 1989. Sedimentology and clast fabric of subaerial debris flow facies in a glacially-influenced alluvial fan: a discussion. Sedimentary Geology 65, 3059-3071.

Cohen, K.M., Gibbard, P., 2010. Global Chronostratigraphical Correlation Table for the Last 2.7 Million Years. Subcommission on Quaternary Stratigraphy (International Commission on Stratigraphy), Cambridge, England.

Colombo, F., Busquets, P., Ramos, E., Vergés, J., Ragona, D., 2000. Quaternary alluvial terraces in an active tectonic region: the San Juan River Valley, Andean Ranges, San Juan Province, Argentina. Journal of South American Earth Sciences 13, 611-626.

Costa, C.H., Gardini, C.E., Diedrix, H., Cortes, J.M., 2000. The Andean orogenic front at Sierra de Las Penas-Las Higueras, Mendoza, Argentina. Journal of South American Earth Sciences 13, 287-292.

Costa, C.H., Audemard, F.A., Bezerra, F.H.R., Lavenu, A., Machette, M.N., Paris, G. 2006. An overview of the Quaternary deformation of South America. Revista de la Asociación Geológica Argentina 61, 461-479.

D’Antonio, H., 1983. Pollen analysis of gruta del Indio. Quaternary of South America and Antarctic Peninsula 1, 83-104.

De Francesco, C.G., Zárate, M.A., Miguel, S.E., 2007. Last Pleistocene mollusk assemblages and inferred paleoenvironments from the Andean piedmont of Mendoza, Argentina. Palaeogeography, Palaeoclimatology, Palaeoecology 251, 461-469.

Denny, C.S., 1965. Alluvial Fans in the Death Valley Region, California and Nevada. United States Geological Survey. Professional Paper 466, 62.

Desilets, D., Zreda, M., 2003. Spatial and temporal distribution of secondary cosmicray nucleon intensities and applications to in situ cosmogenic dating. Earth and Planetary Science Letters 206, 21-42.

Desilets, D., Zreda, M., Prabu, T., 2006. Extended scaling factors for in situ cosmogenic nuclides: new measurements at low latitude. Earth and Planetary Science Letters 246, 265-276.

Dorn, R.I., 2009. The role of climatic change in alluvial fan development. In: Parsons, A.J., Abrahams, A.D. (Eds.), Geomorphology of Desert Environments Part VIII. Chapman \& Hall, London, pp. 723-742.

Dortch, J.M., Owen, L.A., Haneberg, W.C., Caffee, M.W., Dietsch, C., Kamp, U., 2009. Nature and timing of large landslides in the Himalaya and Transhimalaya of northern India. Quaternary Science Reviews 28, 1-18.

Dunai, T.J., 2001. Scaling factors for production rates of in situ produced cosmogenic nuclides: a critical reevaluation. Earth and Planetary Science Letters 176, 157-169.

Fielding, E.J., Jordan, T.E., 1988. Active deformation at the boundary between the Precordillera and Sierras Pameanas, Argentina, and comparison with ancient Rocky Mountain deformation. In: Schmidt, C.I.J., Perry, W.J. (Eds.), Interactions of the Rocky Mountain Foreland and the Cordilleran Thrust Belt. Geological Society of America Memoir, vol. 171, pp. 143-163. Boulder, Colorado.

FAO-IIASA Agro-ecological Zone (AEZ), 2002. http://www.fao.org/nr/land/ databasesinformation-systems/aez-agro-ecological-zoning-system/en/.

FAO AQUASTAT database, 2013. Food and Agriculture Organization of the United Nations (FAO). http://www.fao.org/nr/water/aquastat/data/query/index.html.

Frankel, K.L., Dolan, J.F., Finkel, R.C., Owen, L.A., Hoeft, J.S., 2007. Spatial variations in slip rate along the Death Valley-Fish Lake Valley fault system determined from LiDAR topographic data and cosmogenic ${ }^{10} \mathrm{Be}$ geochronology. Geophysical Research Letter 34, 1-6.

Frankel, K.L., Dolan, J.F., Owen, L.A., Ganey, P., Finkel, R.C., 2011. Spatial and temporal constancy of seismic strain release along an evolving segment of the 
Pacific-North America plate boundary. Earth and Planetary Science Letters 304, 565-576.

Frostick, L.E., Reid, I.A.N., 1989. Climatic versus tectonic controls of fan sequences: lessons from the Dead Sea, Israel. Journal of the Geological Society 146, 527-538.

García, A., Zárate, M., Paez, M.M., 1999. The Pleistocene/Holocene transition and human occupation in the Central Andes of Argentina: Agua de le Cueva locality. Quaternary International 53, 43-52.

Gilli, A., Anselmetti, F.S., Ariztegui, D., Bradbury, J.P., Kelts, K.R., Markgraf, V., McKenzie, J.A., 2001. Tracking abrupt climate change in the Southern Hemisphere: a seismic stratigraphic study of Lago Cardiel, Argentina ( $\left.49^{\circ} \mathrm{S}\right)$. Terra Nova $13,443-448$.

Gimenez, J.C., Lentini, E.J., Cirelli, A.F., 2010. Forecasting Streamflows in the San Juan River Basin in Argentina. Water and Sustainability in Arid Regions 3, 261-274.

González, G.L., Dunai, T., Carrizo, D., Allmendinger, R., 2006. Young displacements on the Atacama Fault System, northern Chile from field observations and cosmogenic 21Ne concentrations. Tectonics 25, 321-342.

Hall, S.R., Farber, D.L., Audin, L., Finkel, R.C., Meriaux, A.S., 2008. Geochronology of pediment surfaces in southern Peru: implications for Quaternary deformation of the Andean forearc. Tectonophysics 459, 186-205.

Hall, S.R., Farber, D.L., Audin, L., Finkel, R.C., 2012. Recently active contractile deformation in the forearc of southern Peru. Earth and Planetary Science Letters 337-338, 85-92.

Hallet, B., Putkonen, J., 1993. Surface dating of dynamic landforms: young boulders on aging moraines. Science 12, 937-940.

Harvey, A.M., Wigand, P.E., Wells, S.G., 1999a. Response of alluvial fan systems to the late Pleistocene to Holocene climatic transition: contrasts between the margins of pluvial Lakes Lahontan and Mojave, Nevada and California, USA. Catena 36, 255-281.

Harvey, A.M., Silva, P.B., Mather, A.E., Goy, J.L., Stokes, M., Zazo, C., 1999b. The impact of Quaternary sea-level and climatic change on coastal alluvial fans in the Cabo de Gata ranges, southeast Spain. Geomorphology 28, 1-22.

Harvey, A.M., Wells, S.G., 2003. Late Quaternary variations in alluvial fan sedimentologic and geomorphic processes, Soda Lake basin, eastern Mojave Desert, California. In: Special Papers Geological Society of America, vol. 368, pp. 207-230.

Harvey, A.M., Mather, A.E., Stokes, M., 2005. The response of dry-region alluvial fans to late Quaternary climatic change. In: Alsharan, A.S., Wood, W.W., Goudie, A.S. Fowler, A., Abdellatif, E.M. (Eds.), Desertification in the Third Millenium. Balkema, Rotterdam, pp. 83-98.

Hetzel, R., Niedermann, S., Tao, M., Kubik, P.W., Ivy-Ochs, S., Gao, B., Strecker, M.R., 2002. Low slip rates and long-term preservation of geomorphic features in Central Asia. Nature 417, 428-432.

Hidy, A.J., Gosse, J.C., Pederson, J.L., Mattern, J.P., Finkel, R.C., 2010. A geologically constrained Monte Carlo approach to modeling exposure ages from profiles of cosmogenic nuclides: an example from Lees Ferry, Arizona. Geochemistry Geophysics Geosystems 11, 18.

International Institute for Applied Systems Analysis (IIASA), 2002. http://www.iiasa. ac.at.

Jordan, T.E., Isacks, B.L., Allmendinger, R.W., Brewer, J.A., Ramos, V.A., Ando, C.J., 1983. Andean tectonics related to geometry of subducted Nazca plate. Geological Study of America Bulletin 94, 341-361.

Kandinsky-Cade, K., Reilinger, R., Isacks, B., 1985. Surface deformation associated with the November 23,1977, Caucete, Argentina, earthquake sequence. Journa of Geophysical Research 90, 12691-12700.

Kesel, R.H., Spicer, B.E., 1985. Geomorphic relationships and ages of soils on alluvial fans in the Rio general valley, Costa Rica. Catena 12, 149-166.

Kochel, R.C., 1990. Humid alluvial fans of the Appalachian Mountains. In: Rachoki, A.H., Church, M. (Eds.), Alluvial Fans: A Field Approach. Wiley, Chichester, pp. 109-129.

Kostaschuk, R.A., Macdonald, G.M., Putnam, P.E., 1986. Depositional processes and alluvial fan-drainage basin morphometric relationships near Banff, Alberta, Canada. Earth Surface Processes and Landforms 11, 471-484.

Kohl, C.P., Nishiizumi, K., 1992. Chemical isolation of quartz for measurement of in-situproduced cosmogenic nuclides. Geochimica et Cosmochimica Acta 56, 3583-3587.

Kröhling, D.M., 1999a. Upper Quaternary geology of the lower Carcarañá Basin, North Pampa, Argentina. Quaternary International 57-58, 135-148.

Kröhling, D.M., 1999b. Sedimentological maps of the typical loessic units in North Pampa, Argentina. Quaternary International 62, 49-55.

Lal, D., 1991. Cosmic ray labeling of erosion surfaces: in situ nuclide production rates and erosion models. Earth and Planetary Science Letters 104, 424-439.

Lifton, N.A., Jull, A.J.T., Quade, J., 2001. A new extraction technique and production rate estimate for in situ cosmogenic ${ }^{14} \mathrm{C}$ in quartz. Geochimica et Cosmochimica Acta 65, 1953-1969.

Lifton, N.A., Bieber, J.W., Clem, J.M., Duldig, M.L., Evenson, P., Humble, J.E., Pyle, R. 2005. Addressing solar modulation and long-term uncertainties in scaling secondary cosmic rays for in situ cosmogenic nuclide applications. Earth and Planetary Science Letters 239, 140-161.

Mancini, M.V., Paez, M.M., Prieto, A.R., Stutz, S., Tonello, M., Vilanova, I., 2005. MidHolocene climatic variability reconstruction from pollen records (32-52 S, Argentina). Quaternary International 132, 47-59.

Markgraf, V., 1983. Late and postglacial vegetational and paleoclimatic changes in subantarctic, temperate, and arid environments in Argentina. Palynology 7, 43-70.
Markgrav, V., 1989. Paleoclimates in Central and South America since 18000 BP based on pollen and lake-level records. Quaternary Science Reviews 8, 1-24.

Markgraf, V., 1993. Paleoenvironments and paleoclimtes in Tierra del Fuego and southernmost Patagonia, South America. Palaeogeography, Palaeoclimatology, Palaeoecology 102, 53-68.

Markgraf, V., Bradbury, J.P., Schwalb, A., Burns, S.J., Stern, C., Ariztegui, D., Gilli, A., Anselmetti, F.S., Stine, S., Maidana, N., 2003. Holocene paleoclimates of southern Patagonia: limnological and environmental history of Lago Cardiel, Argentina. The Holocene 13, 581-591.

Mehl, A.E., Zárate, M.A., 2012. Late Pleistocene and Holocene environmental and climatic conditions in the eastern Andean piedmont of Mendoza $\left(33^{\circ}-34^{\circ} \mathrm{S}\right.$, Argentina). Journal of South American Earth Sciences 37, 41-59.

Meigs, A.J., Krugh, W.C., Schiffman, C., Vergés, J., Ramos, V.A., 2006. Refolding of the thin-skinned thrust sheets by active basement-involved thrust faults in the Eastern Precordillera of Western Argentina. Revista de la Asociación Geológica Argentina 61, 589-603.

Meigs, A.J., Nabelek, J., 2010. Crustal-scale pure shear foreland deformation of western Argentina. Geophysical Research Letters 37, 5.

Meigs, A.J., 2012. Personal communication, September 21st 2012.

Mejdahl, V., 1979. Thermoluminescence dating: beta attenuation in quartz grains. Archaeometry 21, 61-73.

Morales, M., Barberena, R., Belardi, J.B., Borrero, L., Cortegoso, V., Duran, V., Guerci, A., Goni, R., Gil, A., Neme, G., Yacobaccio, H., Zaraté, M., 2009. Reviewing human-environment interactions in arid regions of southern South America during the past 3000 years. Palaeogeography, Palaeoclimatology, Palaeoecology 281, 283-295.

Mpodozis, C., Ramos, V.A., 1989. The Andes of Chile and Argentina. In: Ericksen, G.E. Cañas Pinochet, M.T., Reinemund, J.A. (Eds.), Geology of the Andes and its Relation to Hydrocarbon and Mineral Resources. Circumpacific Council for Energy and Mineral Resources, Earth Sciences Series, vol. 11, pp. 59-90.

Murray, A.S., Wintle, A.G., 2000. Luminescence dating of quartz using an improved single-aliquot regenerative-dose protocol. Rad. Meas. 32, 57-73.

Nishiizumi, K., Imamura, M., Caffee, M.W., Southon, J.R., Finkel, R.C., McAninch, J., 2007. Absolute calibration of $10 \mathrm{Be}$ AMS standards. Nuclear Instruments and Methods in Physics Research 258, 403-423.

Owen, L.A., Finkel, R.C., Haizhou, M., Barnard, P.I., 2006. Late Quaternary landscape evolution in the Kunlun Mountains and Qaidam Basin, Northern Tibet: a framework for examining the links between glaciations, lake level changes and alluvial fan formation. Quaternary International 154-155, 73-86.

Perucca, L.P., Paredes, J.D., 2002. Peligro sísmico en el departamento Albardón y su relación con el área de fallamiento La Laja, provincia de San Juan. Revista de la Asociación Geológica Argentina 57, 45-54.

Portenga, E.W., Bierman, P.R., 2011. Understanding Earth's eroding surface with 10Be. GSA Today 21 (8), 4-10.

Powell, R., Hergt, J., Woodhead, J., 2002. Improving isochron calculations with robust statistics and the bootstrap. Chemical Geology 185, 191-204.

Prescott, J.R., Hutton, J.T., 1994. Cosmic ray contributions to dose rates for luminescence and ESR dating: Large depths and long-term time variations. Rad. Meas. 23, 497-500.

PRIME Laboratory, 2010. Important note concerning ${ }^{10} \mathrm{Be}$ results. http://www. physics.purdue.edu/primelab/News/news0907.php.

Putkonen, J., Swanson, T., 2003. Accuracy of cosmogenic ages for moraines. Quaternary Research 59, 255-261.

Ramos, V.A., Jordan, T.E., Allmendinger, R.W., Mpodozis, C., Kay, S.M., Cortés, J.M., Palma, M., 1986. Paleozoic terranes of the central Argentine-Chilean Andes. Tectonics 5, 855-880.

Ramos, V.A., Cristallini, E.O., Peréz, D.J., 2002. The Pampean flat-slab of the Central Andes. Journal of South American Earth Sciences 15, 59-78.

Ritter, J.B., Miller, J.R., Enzel, Y., Wells, S.G., 1995. Reconciling the roles of tectonism and climate in Quaternary alluvial fan evolution. Geology 23, 245-248.

Rodés, A., Pallàs, R., Braucher, R., Moreno, X., Masana, E., Bourlés, D.L., 2011. Effect of density uncertainties in cosmogenic ${ }^{10} \mathrm{Be}$ depth-profiles: dating a cemented Pleistocene alluvial fan (Carboneras Fault, SE Iberia). Quaternary Geochronology 6, 186-194.

Seong, Y.B., Owen, L.A., Yi, C.L., Finkel, R.C., 2009. Quaternary glaciations of Muztag Ata and Kongur Shan: evidence for glacier response to rapid climate changes throughout the Late Glacial and Holocene in westernmost Tibet. Geological Society of America Bulletin 121, 348-365.

Schmidt, S., Hetzel, R., Kuhlmann, J., Mingorance, F., Ramos, V.A., 2011. A note of caution on the use of boulders for exposure dating of depositional surfaces. Earth and Planetary Science Letters 302, 60-70.

Schmidt, S., Tsukamoto, S., Salomon, E., Frechen, M., Hetzel, R., 2012. Optical dating of alluvial fan deposits at the orogenic front of the Andean Precordillera (Mendoza, Argentina). Geochronometria 39, 62-75.

Schoenbohm, L.M., 2008. Personal communication, December 3rd 2008.

Siame, L.L., Bourles, D.L., Sebrier, M., Bellier, O., Castano, J.C.C., Araujo, M., Perez, M. Raisbeck, G.M., Yiou, F., 1997. Cosmogenic dating ranging from 20 to $700 \mathrm{ka}$ of a series of alluvial fan surfaces affected by the El Tigre fault, Argentina. Geology 25, 975-978.

Siame, L.L., Bellier, O., Sebrier, M., Bourles, D.L., Leturmy, P., Perez, M., Araujo, M., 2002. Seismic hazard reappraisal from combined structural geology, geomorphology and cosmic ray exposure dating analyses: the Eastern Precordillera thrust system (NW Argentina). Geophysical Journal International 150, 241-260.

Servicio Meteorológico Nacional (SMN), 2007. Estadísticas meteorológicas. Argentina. 
Smalley, R., Pujol, J., Regnier, M., Chiu, J., Chatelain, J., Isacks, B.L., Araujo, M., Puebla, N., 1993. Basement seismicity beneath the Andean precordillera think-skinned thrust belt and implications for crustal and lithospheric behavior. Tectonics 12, 63-76.

Spelz, R.M., Fletcher, J.M., Owen, L.A., Caffee, M.W., 2008. Quaternary alluvial-fan development, climate and morphologic dating of fault scarps in Laguna Salada, Baja California, Mexico. Geomorphology 102, 578-594.

Spooner, N.A., Questiaux, D.G., Aitken, M.J., 2000. The use of sodium lamps for lowintensity laboratory safelighting for optical dating. Ancient Thermoluminesence $18,45-49$.

Stone, J.O., 2000. Air pressure and cosmogenic isotope production. Journal of Geophysical Research 105, 23753-23759.

Stone, J.O., 2002. Calibration and scaling of cosmogenic nuclide production rates. Geochimica et Cosmochimica Acta 66.

Tripaldi, A., Forman, S.L., 2007. Geomorphology and chronology of Late Quaternary dune fields of western Argentina. Palaeogeography, Palaeoclimatology, Palaeoecology 251, 300-320.

Tripaldi, A., Zárate, M.A., Brook, G.A., Li, G.Q., 2011. Late Quaternary paleoenvironments and paleoclimatic conditions in the distal Andean piedmont, southern Mendoza, Argentina. Quaternary Research 76, 253-263.

Trowbridge, A.C., 1911. The terrestrial deposits of Owens Valley, California. The Journal of Geology 19, 706-747.
Vergés, J., Ramos, V.A., Meigs, A., Cristallini, E., Bettini, F.H., Cortés, J.M., 2007 Crustal wedging triggering recent deformation in the Andean thrust front between 31S and 33S: Sierras Pampeanas-Precordillera interaction. Journal of Geophysical Research: Solid Earth 112.

von Gosen, W., 1992. Structural evolution of the Argentine precordillera: the Rio San Juan section. Journal of Structural Geology 14, 643-667.

Walling, J., Murray, A., Wintle, A., 2000. The single-aliquot regenerative-dose (SAR) protocol applied to coarse-grain feldspar. Radiation Measurements 32, 529-533.

Wasson, R.J., 1977. Last-glacial alluvial fan sedimentation in the Lower Derwent Valley, Tasmania. Sedimentology 24, 781-799.

Wells, S.G., McFadden, L.D., 1987. Influence of late Quaternary climatic changes on geomorphic and pedogenic processes on a desert piedmont, Eastern Mojave Desert, California. Quaternary Research 27, 130-146.

Wendt, I., Carl, C., 1991. The statistical distribution of the mean squared weighted deviation. Chemical Geology: Isotope Geoscience Section 86, 275-285.

Williams, G.E., 1973. Late Quaternary piedmont sedimentation, soil formation and palaeoclimates in arid South Australia. Zeitschrift fur Geomorphologie $17,102-125$.

Zapata, T.R., Allmendinger, R.W., 1996. Growth strata records of instantaneous and progressive limb rotation in the Precordillera thrust belt and Bermejo basin, Argentina. Tectonics 15, 1065-1083. 